\title{
Recent Efforts on Model-Based Simulation of Engineering Problems - Multiphysics and Multiphase Interactions
}

\author{
A DISSERTATION \\ Presented to \\ The Faculty of the Graduate School \\ University of Missouri \\ In Partial Fulfillment \\ of the Requirements for the Degree of \\ Doctor of Philosophy \\ By Youqing Yang \\ Dr. Zhen Chen, Dissertation Supervisor \\ Columbia, Missouri \\ December, 2016
}


The undersigned, appointed by the Dean of graduate school, have examined the dissertation titled

\section{Recent Efforts on Model-Based Simulation of Engineering Problems - Multiphysics and Multiphase Interactions}

presented by Youqing Yang

a candidate for the degree of Philosophy

and hereby certify that in their opinion it is worthy of acceptance.

Zhen Chen

Yuwen Zhang

Sanjeev Khanna

Mani Salim

Glenn Washer 


\section{To my family}




\section{ACKNOWLEDGEMENTS}

I am very grateful to Professor Zhen Chen for his support and valuable guidance through this research. I greatly appreciate Professor Yuwen Zhang for igniting my research interest on laser drilling process simulation. I thank Dr. Ming Song and Dr. Xiangjun Qiu at Metso Minerals Incorporation as they provided their research experience on multiphase flow. Dr. Pengtao Sun at University of Nevada provided careful inspection on the mathematical formula derivation. Dr. Peter Mackenzie-Helnwein provided his research experience in dealing with volume locking. Also, I thank Professors Sanjeev Khanna, Hani Salim, Glenn Washer, and Vellore Golpalatnam for their help during my studies at the University of Missouri.

In addition, I would like to thank Dr. Luming Shen, Yong Gan, Shan Jiang, Yilong Han, and Stephen Robert for their friendship and the academic environment they have shared with me.

This dissertation would never have been completed without the support of my family. My wife, Hongyan Liu, is always supportive and encouraging of my education, and has helped me take care of my children. My two boys, Bowen and Kevin, provided me certain help and rich joys during the study.

Finally, I thank my parents for their lasting love. 


\section{Table of Contents}

ACKNOWLEDGEMENTS $\quad$ ii

LIST OF TABLES $\quad$ vi

LIST OF ILLUSTRATIONS V vii

ABSTRACTS $\quad x$

Chapter 1 Introduction $\quad 1$

1.1 What is simulation-based engineering science? 1

1.2 How does Simulation-Based Engineering Science (SBES) work? 2

1.3 The challenges for Simulation-Based Engineering Science 3

1.4 My efforts in SBES

\section{Part I Multiphysics - Melt Flow and Heat Transfer in Laser Drilling}

Chapter 2 Introduction $\quad 8$

2.1. Laser drilling $\quad 8$

2.2. Current numerical simulations in laser drilling $\quad 8$

Chapter 3. Interface Energy Balance and Governing Equations 13

3.1 Melt convection 13

3.2 Pressure-dependent saturation temperature 15

$\begin{array}{ll}\text { 3.3 Energy balance at solid-melt interface } & 17\end{array}$

$\begin{array}{ll}3.4 \text { Heat conduction in solid } & 18\end{array}$

3.5 Some examples of energy flux rate in solid via heat conduction 22

Chapter 4. Numerical Solutions of the Melt Flow 31

4.1 Boundary layer formulation 31

4.2 Integration method formulation 34

4.3 Solution of the Temperature Field 37 
5.1 Comparison of the model predicts with experimental data 39

5.2 Drill hole evolution $\quad 40$

5.3 Vapor pressure and temperature $\quad 40$

5.4 The contribution of melt flow 41

5.5 Discussions 42

5.6 Summary for the New Laser Drilling Model 43

Part II Multiphase Interaction - Combined MPM and DEM for SolidFluid Interactions

$\begin{array}{ll}\text { 7.2 Momentum equations } & 60\end{array}$

$\begin{array}{ll}7.3 \text { Constitutive equations } & 65\end{array}$

$\begin{array}{ll}7.4 \text { The equation of solid particle motion } & 67\end{array}$

Chapter 8. Discretization of Governing Equations 70

$\begin{array}{ll}\text { 8.1 Material Point Method } & 70\end{array}$

8.2 The interaction force between solid and fluid phase 74

8.3 Numerical solution for the momentum equation 75

$\begin{array}{ll}\text { Chapter 9. Convergence Study } & 78\end{array}$

9.1 The effect of the MPM cell size 80

9.2 The effect of the number of particles per cell 81 
Chapter 10. Verification and Validation

10.1 Water flow after reservoir dam breakage 


\section{LIST OF TABLES}

Table 5.1Thermophysical properties of test material.

Table 8.1 Physical properties and computational parameters.

Table 9.1 Physical properties and computational parameters.

Table 9.2 Physical properties and computational parameters. 


\section{LIST OF ILLUSTRATIONS}

Figure 3.1. Physical model of laser drilling and the coordinate systems used.

Figure 3.2 a) The established temperature at the very beginning of melting; b) the assumed temperature profile; and c) the underestimated temperature at the moment of melting starts.

Figure 3.3 Diffusion of the existed temperature profile.

Figure 3.4 Temperature history at the melting surface due to the diffusion of the existed temperature profile. A) Drawn on a linear time axis, showing the declining effect with time; B) in logarithm axis of time, showing a rising stage at the very beginning. The temperature gradient history at the solid-liquid boundary C) in linear axis of time; D) in logarithm axis of time.

Figure 3.5 Temperature diffusion due to varying boundary condition.

Figure 3.6 Temperature gradient history at the solid-liquid boundary.

Figure 3.7 Exact solution and approximated solutions at the solid-liquid boundary.

Figure 3.8 Flux change due to the curvature of the solid-melt interface.

Figure 5.1 Comparison of predicted and experimental material removal rates using models of rising-fall profiles.

Figure 5.2 Drill hole profiles evolution predicted from a model assuming rising-fall radial velocity profile with peak velocity at $\xi_{p}=1$. 
Figure 5.3. Pressure profiles at $\tau=\tau_{p}$ with varying laser beam intensity, predicted from a model assuming rising-fall radial velocity profile with peak velocity at $\xi_{p}=1$. The laser beam intensity is given at the center with unit $10^{10} \mathrm{w} / \mathrm{m}^{2}$.

Figure 5.4. Temperature profiles at $\tau=\tau_{p}$ with varying laser beam intensity, predicted from a model that assumes a rising-fall radial velocity profile with a peak velocity at $\xi_{p}=1$. The laser beam intensity is given at the center with unit $10^{10} \mathrm{w} / \mathrm{m}^{2}$.

Figure 5.5. Peak radial velocity, temperature and pressure history predicted from a model assuming rising radial velocity profile with index $m=2$.

Figure 5.6. Peak radial velocity, temperature and pressure history predicted from a model assuming rising-fall radial velocity profile with peak velocity at $\xi_{p}=0.6$.

Figure 5.7. (A) Melting rate, vaporization rate and the effect of the melt flow on interfacechange near the laser beam center at $\xi=0.0125$ and at $\xi=1$.

(B) The change of flow velocity vertical component at both locations.

Figure 5.8. (A) Melting rate, vaporization rate and the effect of the melt flow on interface change near the laser beam center $\xi=0.0125$ and at $\xi=1$. (B) The change of flow velocity vertical component at both locations, similar to Figure 5.7 but with a Prattle number 1000 times larger.

Figure 7.1. (A) Control volume $V$ with fluid and particles, (B) Fluid volume $V_{f}$, excluding solid volume; modified after.

Figure 9.1. Setting up of the dam-break test for solid-liquid mixture.

Figure 9.2. Snapshots showing the motion of solid-liquid mixture in the physical test.

Figure 9.3. Simulation of two-phase flow in a tank, 4 snapshots from numerical modeling. The dark blue dots stand for the glass beads, while light blue dots for water.

Figure 9.4 Effect of the MPM cell size on the numerical solution for the fluid phase .

Figure 9.5 Effect of the MPM cell size on the numerical solution for the solid phase. 
Figure 10.1. Dam breakage simulation.

Figure 10.2. Dam breakage sequence predicted from a MPM simulation.

Figure 10.3. MPM simulated water wave front and experimental data for dam breakage model.

Figure 10.4. MPM simulated water column height and experimental data for dam breakage model.

Figure 10.5. Simulation of two-phase flow in a tank, 4 snapshots from numerical modeling. The dark blue dots stand for the glass beads, while light blue dots for water.

Figure 10.6. Comparison between numerical results and experimental data for two-phase flow. A) Displacement of the liquid phase; B) Displacement of the solid phase.

Figure 10.7. Comparison between numerical results and physical experiment for rotating jar. The dark blue dots stand for the glass beads, while light blue dots for water. A) Model with non-slip boundary condition; B) Stable state of beads and water in a rotating jar in physical experiment; C) Models with $50 \%$ of sticky boundary condition. 


\title{
Recent Efforts on Model-Based Simulation of Engineering Problems - Multiphysics and Multiphase Interactions
}

\author{
Youqing Yang \\ Dr. Zhen Chen, Dissertation Supervisor
}

\begin{abstract}
Simulation-Based Engineering Science (SBES) is playing a more important role in gaining new knowledge and providing guidance for engineering activities, in particular, in the fields in which time scale and/or spatial scale make physical experiments dramatically expensive or even impossible. The success of SBES heavily relies on the development of algorithms that provide the bridge between the models describing physical and engineered systems and the computational devices that generate the digital representations of simulations. My efforts on the development of algorithms that simulate multiphysics and multiphase flow are presented in this dissertation.
\end{abstract}

The first part of the dissertation describe the algorithms for the multiphysical model that simulates the laser drilling process. During laser drilling, heat conduction, melt flow, and vaporization occur in a very short time period. Vaporization also produces the recoil pressure that drives melt flow and complicates the heat transfer and material removal rate. To get a more realistic picture of the melt flow, a series of differential equations were developed that govern the process from pre-heating to melting and evaporation. In particular, the Navier-Stokes equation governing the melt flow is solved with the use of the boundary layer theory and integral methods. Heat conduction in a solid is investigated by using classic solutions with the corrections that reflect the 
change in boundary condition from constant heat flux to Stefan condition. The dependence of saturation temperature on the vapor pressure is taken into account by using the Clausius-Clapeyron equation. Both constantly rising radial velocity profiles and rising-fall velocity profiles are considered. In spite of the assumed varying velocity profiles, the new model predicts that the drilling hole profiles are very close to each other in a specific super alloy for given laser beam intensity and pulse duration. The numerical results show that the effect of melt flow on material removal can be ignored in some cases. The solutions derived can be applied to new cases to determine the role of melt flow and vaporization on laser drilling profile evolution and to study the solid material removal efficiency.

The second part of this dissertation describes a new method that simulates the interaction between fluid and solid elements. The discrete element method (DEM) has been used to deal with the interactions between solid elements of various shapes and sizes, while the material point method (MPM) has been developed to handle the multi-phase (solid-liquid-gas) interactions involving failure evolution. A combined MPM-DEM procedure is proposed to take advantage of both methods so that the interaction between solid elements and fluid particles in a container could be better simulated. In the proposed procedure, large solid elements are discretized by the DEM, while the fluid motion is computed using the MPM. The contact forces between solid elements and rigid walls are calculated using the DEM. The interaction between solid elements and fluid particles are calculated via an interfacial scheme within the MPM framework. The proposed procedure is illustrated by representative examples. The convergence of numerical solutions and the factors affecting the simulation fidelity is also discussed. 


\section{Chapter 1. Introduction}

Our knowledge about the complex world in which we live mainly comes from observations, theoretical thinking, and experiments. In the majority of our human history, the term of “experiment" means physical experiment only in which a certain set of physical device is involved. These kind of physical experiments meet ever increasing challenges as human beings' activity of explore advances into new fields of much different scales in time and space. In the field of molecular dynamics, the physical movements of atoms and molecules are studied by utilizing the great power of numerical experiments, or in another words, computer simulations, simply because the observation and verification of new ideas at such level of scale become too expensive and sometimes impossible. In the earth sciences, the size and history of this planet makes the physical experiments completely impossible, whether the study subject is a volcano or earthquake. In the industry of mining processing, different machines are designed and operated to reduce the size of ore particles so that valuable minerals can be exposed. Traditionally, these machines are designed via semi-empirical models. Because of the huge amount of electricity consumed by the grinding machines, it is desirable to have more energy-efficient equipment. However, the designers do not have the necessary information to help them reach their goal. The simulation-based engineering science (SBES) is becoming a more and more powerful tool to help engineers and scientists to complete their mission.

\subsection{What is simulation-based engineering science?}

Simulation-Based Engineering Science (SBES) is defined as the discipline that provides the

scientific and mathematical basis for the simulation of engineered systems (NSF 2006). Such systems can be microelectronic devices, automobiles, aircrafts, infrastructures of oilfields, or 
ecosystems. SBES provides the traditional engineering fields with the knowledge and techniques of fields such as computer science, mathematics, and the physical and social sciences. As a result, engineers are better able to predict and optimize systems that affect almost all aspects of our lives and work, including the products we use and export, our environment, our security and safety.

\subsection{How does Simulation-Based Engineering Science (SBES) work?}

It is understood that the world we live does not run randomly. Natural laws work whenever the conditions apply, for example, Fick's laws that describe heat and mass diffusion and Newton's laws of motion that govern the behavior of macro body at moderate speed. These laws can be described in certain mathematical forms. Obtaining solutions to these mathematical equations has never been an easy task, even for well-trained mathematicians. The invention of the electronic computer, however, opened a new field to solve mathematical problems with ease. As an example, the motion and deformation of a continuum can be described in terms of partial differential equations. Prior to the invention of the computer, it was not possible to make a prediction of the future motion of a rocket after it leaves the launch device, even if the accurate mathematical model was well known. By inputting a certain set of numerical approaches, the real time change of an object can be predicted and presented to readers after millions and billions of calculations are run in a computer. Computer simulation allows engineers the ability to tell the performance of a product design before the product is actually manufactured. Computer simulation offers may opportunities in sciences. It represents an extension of theoretical science since it is based on mathematical models. These models can be used to test hypothesis since the models characterize the physical predictions. Simulation also provides a powerful alternative to the techniques of experimental science and observation in cases in which phenomena are not observable or when measurements are impractical or too expensive. 


\subsection{The challenges for Simulation-Based Engineering Science}

SBES is powerful, but it is far from perfect. While a computer is necessary equipment, algorithms are the key to solve the problems in SBES. It is the algorithms that convert mathematical models into computational processes. Algorithms provide a bridge between the models describing physical and engineered systems and the computational devices that generating the digital representations of simulations. For many classic problems with simple requirements, reliable algorithms have been developed as a result of many years of trial, verification, and validation. New algorithms are continually needed because we live in an ever-changing, dynamical world in which new problems continuously develop; we don't have any effective algorithms for the new problems just identified or old problems of deeper concerns. As an example, let us consider the mining industry. The ore particles are treated as discrete elements in current simulation technology. Each individual element follows Newton's second law of motion. Current algorithms are able to handle up to several million particles. As the particle size decreases, the number of particles increases exponentially. A cubic meter of ore can be 1000 particles of size $0.1 \mathrm{~m}$, or $10^{9}$ particles of size 1 millimeter, or $10^{18}$ particles of size 1 micron. The number eliminates all attempts to realistically predict the effect of fine particles.

Algorithms vary according to the processes they handle. The algorithms applied in computational fluid dynamics are not the same as those applied in solid structural analysis. In the last decades there has been many changes in simulation tools for simulating crustal deformation in earth science. A linear elastic model may help scientists to find the cause of present-day tectonic stress like photoelastic experiments, but a viscoelastic model or elastic-plastic-viscous model works better for time-dependent processes such as post-glacial rebound, post-seismic stress relaxation (Liu, Yang et al. 2000). For the simulation of the crustal deformation in geological time, 
which includes over ten million years of history, the distortion of finite element mesh posed a threat that it could ruin the reputation of simulation for poor accuracy quality. This problem was finally solved by exploiting remesh technique (Yang and Liu 2009, Yang and Liu 2013). Later, it was shown that there are many meshless methods that provide alternative solutions for nonlinear problems (Nguyen, Rabczuk et al. 2008). Some algorithms provide solutions for more difficult problems like penetration and explosions.

In addition to the development of mathematically correct algorithms, there is the challenge that comes from simulation code verification and validation (Oberkampf and Blottner 1998). Simulation code verification and validation answer the question of how reliable and accurate the simulation is when they are both run correctly. Code verification is a prerequisite to code validation. The former requires that the code is mathematically correct. If there exists an analytic solution, the code should be able to produce a numerical result that approximates to the analytic solution at a certain convergence rate. The latter requires that the code can reproduce the numerical result that is close to the result of physical experiments. Once the code passes the validation, the confidence to predict real world events is then established. As the simulated subject becomes much complicated and difficult, such validation may be completed step by step. The motion of ore particles in an industrial mill is invisible and hard to monitor, but the power drawn by the mill is a good indicator that can be compared with the theoretical value predicted in numerical experiments. The solid material flow rate is a macro variable that can be measured, but the current discrete element method and the corresponding algorithms seem unable to predict due to the existence of fine particles and complicated mill structures. For the purpose of validation, a transparent pilot mill can be manufactured to laboratory scale. Thus, it is predictable that the development of 
algorithms and the feedback from code verification are necessary steps to mature the simulation tools and they should not be expected to be finished over one night.

Keep in mind that all models are an approximations to our real world. As our study proceeds to a new level, appropriate algorithms must be developed to help us to reach our goals. Therefore, continuous effort to develop new algorithms is required so as to advance the SBES.

\subsection{My efforts in SBES}

In this dissertation, my efforts will be directed in the following way. Two topics will be presented: the simulation on multiphysics and the simulation on multiphase flow. The primary results from the first topic has been published (Yang, Chen et al. 2016) and the results from the second topic have been accepted for publication (Yang, Sun et al. 2017). The two topics are in different engineering fields, but fundamentally they are in the same catalogue. They are real engineering problems and are governed by the conservational laws described in partial differential equations. They are able to be solved by numerical methods borrowed from mathematics and implemented with the aid of computer technology. As a scholar in computational mechanics, I present these topics as a whole to the committee.

In the multiphysics study, my major contribution is the derivation of the solution for the Navier-Stokes equation in a cylindrical coordinate system that governs the melt flow in laser drilling process. In previous numerical studies, the influence of melt flow was assumed to be free stream or simply neglected without justification. Thus, explicitly including viscous melt flow will significantly improve the quality of simulation. I also derived the exact solution for heat conduction in the solid before and after melt occurs. A more realistic relationship between saturation temperature and vapor pressure is included in my model, as well as latent and explicit 
heat transfer in the solid-liquid interface, liquid-vapor interface. The model provides a general tool for laser drilling simulation and provides evidence that melt flow is ignorable in a specific case.

In the multiphase flow simulation, an accurate differential equation was derived that reflects the current simulation practice using few assumptions. More importantly, a set of numerical approaches was developed for the first time to solve the new equation based on the Material Point Method (MPM) and Discrete Element Method (DEM), referred to as the combined MPM-DEM method in this study. The new numerical approach is implemented in $\mathrm{C}++$ codes. Verification and validation was conducted in three cases, including solid-fluid interactions. Convergence study shows very good performance of the new approach. The comparison with physical experimental data shows this new method is applicable for more general simulation of solid-fluid interaction.

The rest of this dissertation is organized as follows. The study on melt flow and heat transfer is given in the first section, in which the derivation of the governing equations, numerical solutions, and simulation results will be presented one by one. The second part contains the multiphase phase flow simulation. Similarly, the governing equations, institutive equations, numerical solutions, model verification, and validation are given. Conclusions for each part are drawn at the end respectively. 


\section{Part I. Multiphysics}

Simulation of Melt Flow and Heat Transfer in Laser Drilling 


\section{Chapter 2. Introduction of Numerical simulations in laser drilling}

\subsection{Laser drilling}

Laser drilling is a process of creating a hole in a material by pulsing a laser beam on a particular surface area of the material. The laser beam is of high density of energy that causes the material to melt and vaporize. To make better control on this new technology, it is critical to quantitatively know the energy density level and the laser pulse duration in the first step. Melt recast layers, melt drop spatters occur during the rapid phase change process. It is necessary to have such knowledge in order to have a good quality of the product. From a scientific point of view, the new field of applying laser technology to industrial purposes introduces new opportunities to probe deeper into a wider variety of the phenomena encountered in laser-matter interaction. The phenomena are related to physical models describing energy propagation and absorption, ionization, evaporation, and nonlinear transport of mass, momentum, and energy (Parandoush and Hossain 2014).

\subsection{Current numerical simulations in laser drilling}

There are two major mechanisms that control the material removal in the process of laser drilling: (1) melt evaporation, and (2) melt expulsion by the vaporization-induced recoil force. It is generally believed that melt removal will dominate if an assisting gas is applied on the melt surface when the melt surface temperature does not significantly exceed the melting point and the evaporation rate is low enough not to produce a noticeable recoil pressure. In the cases where there is no assisting gas involved, melt expulsion varies with the recoil pressure, which is highly dependent on the surface temperature. At a high surface temperature, melt removal due to evaporation may exceed that by the hydrodynamic mechanism due to the recoil force. In an early 
simulation, a significant portion of the absorbed laser intensity was found to be taken away by the melt flow from the heat interaction zone (Semak and Matsunawa 1997). Due to the difficulty in directly measuring the geometry of the interfaces and the temperature and recoil pressure at the melt-vapor interface, the portion of melt removed by the recoil force driven flow is hard to verify with existing experimental capabilities. The majority of numerical studies on laser drilling have simply neglected the effect of the melt flow on material removal without justification.

Considerable research has been carried out to develop a theoretical model for predicting laser drilling response. Assuming a constant laser beam intensity profile, von Allmen analyzed the drilling velocity and drilling efficiency by using a one-dimensional (1-D) transient gas dynamic model (Allmen 1976). Chan and Mazumder (Chan and Mazumder 1987) developed a 1-D steady state model to incorporate liquid expulsion, but the 1-D assumption is not suited for hole drilling with high aspect ratio and the drilling process is transient. Kar and Mazumder (Kar and Mazumder 1990) extended the model to 2-D, in which melt expulsion was not considered. Armon et al. formulated a 1-D metal drilling problem based on the enthalpy balance method and solved the problem by using the Crank-Nicholson method (Armon, Zvirin et al. 1989). They also conducted

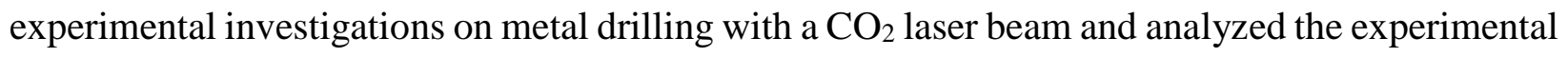
results by using their theoretical model (Armon, Hill et al. 1989). A more rigorous treatment of melt expulsion was presented by Ganesh et al.(Ganesh, Faghri et al. 1997); this was a 2-D transient generalized model, which incorporated conduction, convection, and phase change heat transfer during laser drilling; this model, however, is computationally demanding. Zhang and Faghri developed an analytical model to study the effect of solid conduction on the material removal rate and phase change at interfaces (Zhang and Faghri 1999). In this model, the effect of melt flow on heat transfer is neglected. Zhang et al. developed a 2-D transient model in which a Knudsen layer 
is considered at the melt-vapor front, but the effect of melt flow is not considered (Zhang, Yao et al. 2001). Pastras et al. analyzed material removal efficiency by assuming linear temperature profiles in solid, liquid, and vapor (Pastras, Fysikopoulos et al. 2014), implicitly assuming that melt flow does not cause any disturbance on temperature gradient.

The melt flow has been investigated by some existing models. For example, the model developed by Semak and Matsunawa (Semak and Matsunawa 1997) and a later version adapted by Low et al. (Low, Li et al. 2002) to include the effect of melt flow with an assisting gas on laser drilling are both integral, steady-state, and analytical models based on mass and energy conservation. Semak and Matsunawa attempted to evaluate the effect of recoil pressure during the melt ejection process. Their model is based on the assumption of a free flow layer of melt under the laser beam of hat-top shaped intensity profile (Semak and Matsunawa 1997). Ng et al. developed a model of laser drilling that incorporated the effect of using oxygen as an assisting gas. They assumed that the melt front propagates with an averaged velocity and the averaged melt thickness is determined by dividing the thermal diffusivity of the melt with the averaged propagating velocity ( $\mathrm{Ng}$, Crouse et al. 2006). Zeng et al. developed a 2-D analytical model for optical trepanning assuming that vaporization rate is negligible (Zeng, Latham et al. 2005). Collins and Gremaud developed a simple 1-D model by cross section averaging while neglecting the contribution of the radial flow velocity component (Collins and Gremaud 2011). It is worthy to note that the melt flow models developed by Allmen, Semak and Matsunawa, Ng et al. (Allmen 1976, Semak and Matsunawa 1997, Ng, Crouse et al. 2006) and the latest simulation by Semak and Miller (Semak and Miller 2013) all assume a hat-top-shaped intensity profile. The assumption about the laser beam intensity profile directly affects the conclusion about the melt flow (Han and Liou 2004, Shuja and Yilbas 2011, Momin, Shuja et al. 2012). Using the hat-top profile, the melt 
surface temperature could be assumed to be constant, although rapid change occurs at the margin of the melt. If the melt flow is further assumed to be free of shear traction, the recoil pressure can also be assumed to be constant; this assumption definitely leads one to overestimate the role of melt expulsion. Hence, the role of the melt flow in laser drilling needs to be reevaluated based on a more realistic model.

A more realistic model should consider vaporization based on real physics. It is well known that vaporization occurs at any temperature above the melting point, and the recoil pressure is highly dependent on melt surface temperature. However, some previous models assumed a Stefan condition at the melt-vapor interface (Ganesh, Faghri et al. 1997), while others took the boiling point for the liquid-vapor transition (Zhang, Shen et al. 2014). Solana et al. assumed the recoil pressure of the Gaussian form (Solana, Kapadia et al. 2001). Li et al. assumed that liquid-vapor transition takes place over a certain temperature range ( $\mathrm{Li}, \mathrm{Li}$ et al. 2004).

How to simulate heat conduction more accurately is also important in better predicting the real physics. Heat conduction in solid is a classic problem, but the heat conduction in laser drilling involves a change in boundary condition, which has led to different approaches by different investigators. Earlier researchers assumed a constant melt layer thickness and a constant melting rate, and consequently developed a steady state heat conduction model (Chan and Mazumder 1987). Modest derived a transient heat conduction model by assuming that the phase change from solid to vapor occurs in a single step (Modest 1996). By assuming a parabolic temperature profile and applying integration, the partial differential equation was transformed into an ordinary differential equation, which was later applied to an integral solution by Zhang and Faghri (Zhang and Faghri 1999). Shen et al. also derived a transient heat conduction model by assuming a temperature profile of exponential function (Shen, Zhang et al. 2001). Ho and Lu developed a 
transient heat conduction model by adding a heat source term in the solid to represent the energy flux from the laser beam (Ho and Lu 2003). Shidfar et al. developed a transient heat conduction model by assuming that the solid being heated is initially at the melting point (Shidfar, Alinejadmofrad et al. 2009). These models have been frequently cited in the laser simulation research community and have helped in understanding the physics with different degrees of success, but they have all had certain limitations due to assumptions made. Laser drilling in a solid is a transient heat conduction process with thermal energy emanating from laser beams; hence, classical solutions (Rohsenow, Hartnett et al. 1998) are available for us to develop a theoretical model that might better predict the process with few assumptions.

In this work, the aim is to develop a theoretical model with the melt flow effect to be explicitly included. Once the solution regarding melt flow velocity becomes available, the effect of melt flow on the heat transfer can be evaluated with confidence. The assumption of ignoring melt flow can be verified or justified using the proposed method. It is intended to keep the model as simple as possible to reduce computational expense. Unlike previous studies, the melt is assumed to behave like a Newtonian fluid, and the non-slip boundary condition is applied along the solid-liquid interface. With a Gaussian intensity profile being assumed for the laser beam, the vapor temperature, recoil pressure, and initial melting time will vary in the radial direction. Heat absorbed in the solid and transferred by phase change and melt flow will be taken into account. Both boundary layer formulation and integral forms of momentum equation and energy equation will be developed. Finally, a set of numerical experiments will be performed for a special super alloy by using the formula developed in this study. 


\section{Chapter 3. Interface energy balance and governing equations}

Figure 1 shows the coordinate system used for formulating the equations, in which $r$ denotes the radial direction, and $Z$ the upward direction pointing to the melt from the solid, with origin at the solid-melt interface. Note that we use the lower-case letter $z$ to mark the change of interface between phases, and $z$ originates at the initial solid surface and always points toward the solid. The solid-melt interface is curved in nature, but for the first order of approximation, the difference between tangent direction and radial direction in current study on melt flow is simply ignored, similar to approach by Ganesh et al.(Ganesh, Faghri et al. 1997). The local coordinate $n$ is defined to originate at the solid-melt interface and point to the solid in the normal direction.

\subsection{Melt convection}

In the melt layer, the vertical velocity is much smaller than the lateral component, which enables the simplification of the governing equations considerably. We also ignore the friction produced heat, surface tension, and gravitational force. Similar to the assumptions in the jet impingement study by Kendoush (Kendoush 1998), the continuity equation, momentum equation, and energy equation can be written as:

$$
\begin{aligned}
& \frac{\partial u}{\partial r}+\frac{\partial v}{\partial Z}+\frac{u}{r}=0 \\
& u \frac{\partial u}{\partial r}+v \frac{\partial u}{\partial Z}=\frac{\mu}{\rho}\left[\frac{\partial^{2} u}{\partial Z^{2}}+\frac{1}{r} \frac{\partial u}{\partial r}-\frac{u}{r^{2}}\right]-\frac{1}{\rho} \frac{\partial p}{\partial r} \\
& \frac{\partial T_{l}}{\partial t}+u \frac{\partial T_{l}}{\partial r}+v \frac{\partial T_{l}}{\partial Z}=\alpha_{l} \frac{\partial^{2} T_{l}}{\partial Z^{2}}
\end{aligned}
$$

where $u$ and $v$ are the radial, and vertical velocity component, respectively. $\rho$ represents the density of the melt and $\mu$ the viscosity. $T_{l}$ is the temperature in the liquid and $\alpha_{l}$ is the thermal diffusivity. The lower case $t$ denotes time and $p$ represents pressure. For the convenience of the 
description of boundary layer theory, $U$ and $V$ stand for free stream flow velocity outside of the boundary layer, and the radial component $U$ does not change in the Z-direction. From Bernoulli's principle, we have the following equation for the free stream,

$$
-\frac{1}{\rho} \frac{\partial p}{\partial r}=U \frac{d U}{d r}
$$

Considering that the melt layer is much thinner compared with its lateral dimension, we assume that the pressure variation in the thickness direction is ignorable; thus, the second momentum conservation equation becomes $\frac{\partial p}{\partial Z}=0$, so pressure can be treated as a function of radius only. Defining $\xi=\frac{r}{R^{\prime}} \zeta=\frac{Z}{R^{\prime}}, \tau=\frac{t \alpha_{l}}{R^{2}}, \alpha_{l}=\frac{k_{l}}{\rho c_{p l}}, u^{*}=\frac{u R}{\alpha_{l}}, v^{*}=\frac{v R}{\alpha_{l}}, U^{*}=\frac{U R}{\alpha_{l}}, p^{*}=\frac{p R^{2}}{\rho \alpha_{l}{ }^{2}}, \theta_{l}=\frac{T_{l}-T_{m}}{T_{s a t 0}-T_{m}}$, we rewrite the above equations in a non-dimensional form

$$
\begin{aligned}
& \frac{\partial u^{*}}{\partial \xi}+\frac{\partial v^{*}}{\partial \zeta}+\frac{u^{*}}{\xi}=0 \\
& u^{*} \frac{\partial u^{*}}{\partial \xi}+v^{*} \frac{\partial u^{*}}{\partial \zeta}=\operatorname{Pr}\left[\frac{\partial^{2} u^{*}}{\partial \zeta^{2}}+\frac{1}{\xi} \frac{\partial u^{*}}{\partial \xi}-\frac{u^{*}}{\xi^{2}}\right]-\frac{d p^{*}}{d \xi} \\
& \frac{\partial \theta_{l}}{\partial \tau}+u^{*} \frac{\partial \theta_{l}}{\partial \xi}+v^{*} \frac{\partial \theta_{l}}{\partial \zeta}=\frac{\partial^{2} \theta_{l}}{\partial \zeta^{2}} \\
& \quad-\frac{\partial p^{*}}{\partial \xi}=U^{*} \frac{d U^{*}}{d \xi}
\end{aligned}
$$

For convenience, we simply remove the asterisks from each variable now and hereafter. The $-\frac{d p}{d \xi}$ is also replaced with $U \frac{d U}{d \xi}$. Now we have

$$
\begin{aligned}
& \frac{\partial u}{\partial \xi}+\frac{\partial v}{\partial \zeta}+\frac{u}{\xi}=0 \\
& u \frac{\partial u}{\partial \xi}+v \frac{\partial u}{\partial \zeta}=\operatorname{Pr}\left[\frac{\partial^{2} u}{\partial \zeta^{2}}+\frac{1}{\xi} \frac{\partial u}{\partial \xi}-\frac{u}{\xi^{2}}\right]+U \frac{d U}{d \xi}
\end{aligned}
$$




$$
\frac{\partial \theta_{l}}{\partial \tau}+u \frac{\partial \theta_{l}}{\partial \xi}+v \frac{\partial \theta_{l}}{\partial \zeta}=\frac{\partial^{2} \theta_{l}}{\partial \zeta^{2}}
$$

Assuming there exists a boundary layer of melt of thickness $\delta$, we have the boundary conditions as follows

$$
\begin{aligned}
& v=0 \text { at } \zeta=0 \\
& u=U \text { at } \zeta=\delta \\
& \frac{\partial u}{\partial \zeta}=0 \text { at } \zeta=\delta \\
& u=0 \text { at } \zeta=0 \\
& u=0 \text { at } \xi=0 \\
& \theta_{l}=0 \text { at } \zeta=0 \\
& \theta_{l}=\theta_{\text {sat }} \text { at } \zeta=g(\xi, \tau)
\end{aligned}
$$

\subsection{Pressure-dependent saturation temperature}

In the following sessions, $z=s_{1}(r, t)$ and $z=s_{2}(r, t)$ are the vapor-melt interface and meltsolid interface respectively. At $z=s_{1}(r, t)$, energy conservation requires that the sum of vapor kinetic energy, latent heat due to vaporization, and sensible heat into the melt be equal to the input energy from laser beam, i.e.,

$$
\frac{1}{2} j_{v} M V_{v}^{2}+h_{l v} \rho \frac{\partial s_{1}}{\partial t}+\left.k_{l} \frac{\partial T_{l}}{\partial z}\right|_{z=s_{1}}=I
$$

where $M$ is the molar mass of the gas and $j_{v}$ is the molar flux of evaporation, $h_{l v}$ denotes the latent heat of vaporization, and $k_{l}$ represents the heat conductivity of the liquid. $I$ is the laser beam 
intensity. Eq. (3-12) actually is the equilibrium of energy per unit time and per unit area. Assuming the temperature is continuous at the melt-vapor interface, the vapor velocity at the interface is

$$
V_{v}=\sqrt{\gamma R_{g} T_{\text {sat }} / M}
$$

where $\gamma=\frac{c_{p}}{c_{v}}=\frac{5}{3}$ for a monoatomic ideal gas (Bellantone and Ganesh 1991). From Eq. (3-12), one gets

$$
\rho \frac{\partial s_{1}}{\partial t}=\frac{I-\frac{1}{2} j_{v} M V_{v}^{2}-k_{l} \frac{\partial T}{\partial z} l_{z=s_{1}}}{h_{l v}}
$$

The vapor pressure is related to the temperature by the Clausius-Clapeyron equation:

$$
p\left(T_{\text {sat }}\right)=p_{0} \exp \left[\frac{h_{l v}}{R_{g}}\left(\frac{1}{T_{\text {sat } 0}}-\frac{1}{T_{\text {sat }}}\right)\right]
$$

where $T_{\text {sat }}$ denotes the vapor temperature and $T_{\text {sat } 0}$ is the saturation temperature at pressure $p_{0}$. $R_{g}$ is the gas constant. The vapor leaving the melt surface can also be counted by using the product of molar flux and molar mass.

$$
\rho \frac{\partial s_{1}}{\partial t}=j_{v} M
$$

Bellantone and Ganesh (Bellantone and Ganesh 1991) obtained the following relation:

$$
j_{v}=\frac{p}{\sqrt{2 \pi M R_{g} T_{s a t}}}
$$

Substituting Eq. (3-16) into Eq. (3-14), one gets

$$
j_{v}=\frac{I-\left.k_{l} \frac{\partial T_{L}}{\partial z}\right|_{z=s_{1}}}{h_{l v} M+\frac{1}{2} \gamma R_{g} T_{s a t}}
$$

From the right sides of Eq. (3-18) and Eq. (3-17), one has 


$$
p=\sqrt{\frac{2 \pi R_{g} T_{s a t}}{M}} \frac{I-\left.k_{l} \frac{\partial T_{l}}{\partial z}\right|_{z=s_{1}}}{h_{l v}+\frac{\gamma R g}{2 M} T_{s a t}}
$$

Substituting Eq. (3-15) into Eq. (3-19), one gets

$$
p_{0} \exp \left[\frac{h_{l v}}{R_{g}}\left(\frac{1}{T_{\text {sat } 0}}-\frac{1}{T_{s a t}}\right)\right]=\sqrt{\frac{2 \pi R_{g} T_{s a t}}{M}} \frac{I-\left.k_{l} \frac{\partial T_{l}}{\partial z}\right|_{z=s_{1}}}{h_{l v}+\frac{\gamma R g}{2 M} T_{s a t}}
$$

The laser beam intensity takes the Gaussian form as follows

$$
I(r, t)=I_{0} \exp \left(-\frac{r^{2}}{R^{2}}\right)=I_{0} \exp \left(-\xi^{2}\right), 0<t<t_{p}
$$

Using dimensionless parameters $k^{\prime}=\frac{k_{l}\left(T_{s a t 0}-T_{m}\right)}{R I_{0}}$ and $I^{\prime}=\frac{I_{0}}{p_{0} h_{l v}} \sqrt{\frac{2 \pi R_{g} T_{\text {sat0 }}}{M}}, \Lambda=\frac{\gamma R_{g} T_{\text {sat0 }}}{2 M h_{l v}}$, $\theta_{\text {sat }}=\frac{T_{\text {sat }}-T_{m}}{T_{\text {sat } 0}-T_{m}}, \theta_{m}=\frac{T_{m}}{T_{\text {sat } 0}}$, we simplify Eq. (3-20) as

$$
\begin{array}{r}
\left\{1+\Lambda\left[\theta_{\text {sat }}\left(1-\theta_{m}\right)+\theta_{m}\right]\right\} \exp \left[H_{l v}\left(1-\frac{1}{\theta_{\text {sat }}\left(1-\theta_{m}\right)+\theta_{m}}\right)\right]= \\
I^{\prime} \sqrt{\theta_{\text {sat }}\left(1-\theta_{m}\right)+\theta_{m}}\left[\exp \left(-\xi^{2}\right)-\left.k^{\prime} \frac{\partial \theta_{l}}{\partial \zeta}\right|_{\zeta=s_{1}}\right]
\end{array}
$$

\subsection{Energy balance at solid-melt interface}

Both the energy for heating up the solid and melting are from the liquid; thus, the energy balance at the solid-liquid interface can be written as follows:

$$
\rho h_{l s} \frac{\partial s_{2}}{\partial t}=\left.k_{s} \frac{\partial T_{S}}{\partial z}\right|_{z=s_{2}}-\left.k_{l} \frac{\partial T_{l}}{\partial z}\right|_{z=s_{2}}
$$

where $h_{l s}$ denotes the latent heat of melting and $k_{s}$ and $k_{l}$ represent the thermal conductivity of the solid and the fluid, respectively. In this equation, it is assumed that the density of the solid is the same as that of the fluid. The dimensionless form is

$$
\frac{\partial S_{2}}{\partial \tau}=\operatorname{Ste}_{\alpha} N_{c}\left(-\left.\frac{\partial \theta_{s}}{\partial \zeta}\right|_{\zeta=0}+\left.k \frac{\partial \theta_{l}}{\partial \zeta}\right|_{\zeta=0}\right)
$$


where Ste $=\frac{c_{p l}\left(T_{\text {sat }}-T_{m}\right)}{h_{l s}}$ is the Stefan number; $N_{\alpha}=\frac{\alpha_{s}}{\alpha_{l}}$ is the thermal diffusivity ratio; and $N_{c}=$ $\frac{c_{p s}}{c_{p l}}$ is the specific heat ration; $k=\frac{k_{l}}{k_{s}}$ represents the ratio of liquid thermal conductivity over solid.

Eq. (3-14) is the energy balance at the melt-vapor interface. From Eq . (3-13) and (3-17), the vapor kinetic energy $\frac{1}{2} j_{v} M V_{v}^{2}=\frac{p \gamma}{2} \frac{\sqrt{R_{g} T_{s a t}}}{\sqrt{2 \pi M}}=\frac{p \sqrt{\gamma \Lambda h_{l v}}}{2 \sqrt{\pi}} \sqrt{\frac{T_{s a t}}{T_{s a t 0}}}$, and let $\Psi=\frac{\sqrt{\gamma \Lambda}}{2 \sqrt{\pi}} \frac{\alpha_{l}}{R \sqrt{h_{l v}}}$; hence, the dimensionless form of $(3-14)$ is

$$
\frac{\partial s_{1}}{\partial \tau}=N_{i} \exp \left(-\xi^{2}\right)-\Psi p \sqrt{\theta_{s a t}\left(1-\theta_{m}\right)+\theta_{m}}-\left.\frac{h_{l s}}{h_{l v}} S t e \frac{\partial \theta_{l}}{\partial \zeta}\right|_{\zeta=g}
$$

Note that the pressure $p$ in the Eq. (3-25) is dimensionless whereas the one in the (3-15), (3-

17), (3-19) is dimensional.

\subsection{Heat conduction in solid}

To analyze the heat conduction in the solid, we set up a local one-dimensional coordinate system $n$ that originates at the melt-solid interface and points to the solid in the normal direction (see Fig. 1). Before the surface temperature reaches the melting point, the solid surface receives constant heat flux, and the solution is (Rohsenow, Hartnett et al. 1998):

$$
\theta_{s}(\xi, n, \tau)=\theta_{i}+\frac{k}{k^{\prime}} \exp \left(-\xi^{2}\right) \sqrt{N_{\alpha} \tau}\left[\frac{1}{\sqrt{\pi}} \exp \left(-\eta^{2}\right)-\eta \operatorname{erfc}(\eta)\right]
$$

where $\theta_{s}=\frac{T_{S}-T_{m}}{T_{s a t 0}-T_{m}}$ is the dimensionless temperature in the solid whereas $T_{s}$ corresponds to the dimensional value; $\theta_{i}=\frac{T_{i}-T_{m}}{T_{s a t 0}-T_{m}}$ is the dimensionless initial temperature of the solid, and $T_{i}$ is the dimensional value, $T_{m}$ is the melting point, and $k^{\prime}=\frac{k_{l}\left(T_{s a t 0}-T_{m}\right)}{R I_{0}}$, and $\eta=\frac{n}{2 \sqrt{N_{\alpha} \tau}}$, which is referred to as the similarity variable.

It is obvious that the highest temperature is at the surface. When $T_{s}=T_{m}, \theta_{s}=0$, phase change begins at the surface. The melting starting time can thus be obtained from Eq. (3-26)

$$
\tau_{m}=\frac{\pi}{N_{\alpha}}\left[\frac{k^{\prime} \theta_{i}}{k} \exp \left(\xi^{2}\right)\right]^{2}
$$

At the moment $\tau=\tau_{m}$, the following temperature profile is established. 


$$
\theta_{s}\left(\xi, n, \tau_{m}\right)=\theta_{i}-\theta_{i}\left[\exp \left(-\eta_{m}^{2}\right)-\sqrt{\pi} \eta_{m} \operatorname{erfc}\left(\eta_{m}\right)\right]
$$

where $\eta_{m}=\frac{n}{2 \sqrt{N_{\alpha} \tau_{m}}}$.

The exact solution for the time period after melting starts was derived in this study as well as the geometrical correction for the temperature gradient. The exact solution for the time period after melting starts consists of three parts:

$$
\theta_{s}(\xi, n, \tau)=\theta_{s 1}+\theta_{s 2}+\theta_{s 3}, \tau>\tau_{m}
$$

where

$$
\theta_{s 1}(n, \tau)=\theta_{i} \operatorname{erf}\left[\frac{n}{2 \sqrt{N_{\alpha}\left(\tau+\tau_{0}-\tau_{m}\right)}}\right], \tau>\tau_{m}
$$

in which

$$
\tau_{0}=\frac{\left[\frac{\theta_{i} k^{\prime}}{k} \exp \left(\xi^{2}\right)\right]^{2}}{4 N_{\alpha}}=\frac{\tau_{m}}{4 \pi}
$$

Equation (3-30) is the solution of the Stefan problem. The idea of adding $\tau_{0}$ is to avoid singularity at $\tau=\tau_{m}$ and to make the temperature gradient (heat flux) continuous at $\tau=\tau_{m}$, which also has a generated temperature profile

$$
\theta_{s 1}\left(n, \tau_{m}\right)=\theta_{i} \operatorname{erf}\left(\frac{n}{2 \sqrt{N_{\alpha} \tau_{0}}}\right)
$$

This profile is called "generated" because the initial condition $\theta_{i}$ is assumed at the moment $\tau=\tau_{m}-\tau_{0}$ for the Stefan solution. Because the temperature field at melting starting time is not the profile expressed in Eq. (3-32), but the established profile shown in Eq. (3-28). Eq. (3-30) is not the exact solution of the problem concerned. The difference between Eq. (3-28) and (3-32) must be corrected. For a semi-infinity object with no heat source and zero initial and boundary 
conditions, the temperature evolution due to an existing non-zero temperature profile $G(n)$ at a given moment can be calculated using the following formula (Evans 1997)

$$
\theta_{s 2}(n, \tau)=\frac{1}{2 \sqrt{\pi N_{\alpha}\left(\tau-\tau_{m}\right)}} \int_{0}^{\infty} G(y) e^{-\frac{(n-y)^{2}}{4 N_{\alpha}\left(\tau-\tau_{m}\right)}} d y, \tau>\tau_{m}
$$

For the problem $G(n)=\theta_{s}\left(\xi, n, \tau_{m}\right)-\theta_{s 1}\left(n, \tau_{m}\right)$. Obviously this solution will lead to a non-zero boundary temperature at $n=0$.

$$
\theta_{s 2 b}(0, \tau)=\frac{1}{2 \sqrt{\pi N_{\alpha}\left(\tau-\tau_{m}\right)}} \int_{0}^{\infty} G(y) e^{-\frac{y^{2}}{4 N_{\alpha}\left(\tau-\tau_{m}\right)}} d y, \tau>\tau_{m}
$$

In order to keep the constant zero temperature at the boundary (Stefan condition), it is necessary to add a new correction by considering the effect of boundary temperature $\theta_{s 3 b}(0, \tau)=$ $-\theta_{s 2 b}(0, \tau)$. The solution of this Dirichlet condition is classic, and the uniqueness of the solution is proven in (Evans 1997). It can be solved by using numerical methods like finite element methods or finite difference methods. An easy way is to use PDEPE function in MATLAB. The solution is now marked as $\theta_{s 3}$.

Both $\theta_{s 2}$ and $\theta_{s 3}$ decline very fast with time, and they become negligible as $\tau>5 \tau_{m}$. The Stefan solution is the major contributor. To reduce computational expense, an approximated solution is obtained by simply shifting the Stefan solution backwards about $3 \tau_{0}$, which leads to negligible change for $\tau>5 \tau_{m}$, but attains significant improvement on the temperature gradient for $\tau<\tau_{m}$

$$
\begin{aligned}
& \theta_{s}(n, \tau) \approx \theta_{i} \operatorname{erf}\left[\frac{n}{2 \sqrt{N_{\alpha}\left(\tau+3 \tau_{0}-\tau_{m}\right)}}\right] \\
& \frac{\partial \theta_{s}}{\partial n}(0, \tau) \approx \frac{\theta_{i}}{\sqrt{\pi N_{\alpha} \tau_{m}} \sqrt{\frac{\tau}{\tau_{m}}-1+\frac{3}{4 \pi}}}
\end{aligned}
$$


Since the coordinate origin moves at the rate of $V_{n}=\frac{\partial S_{2}}{\partial \tau} / \sqrt{1+\left(\frac{\partial S_{2}}{\partial \xi}\right)^{2}}$, the effective temperature gradient at a static coordinate system will be $\frac{\partial \theta_{s}}{\partial n}(0, \tau)+\theta_{i} \frac{V_{n}}{N_{\alpha}}$ in terms of same energy fluency.

The curvature-corrected temperature gradient is $C_{c}\left[\frac{\partial \theta_{s}}{\partial n}(0, \tau)+\theta_{i} \frac{V_{n}}{N_{\alpha}}\right]$ where

$$
C_{c}= \begin{cases}\frac{a d}{2 \sin ^{-1} \frac{a d}{2}} & a>0 \\ 1 & a=0 \\ \frac{2 \sin ^{-1} \frac{a d}{2}}{a d} & a<0\end{cases}
$$

and

$$
a=-\frac{\partial^{2} S_{2}}{\partial \xi^{2}}\left[1+\left(\frac{\partial S_{2}}{\partial \xi}\right)^{2}\right]^{-3 / 2}
$$

is the curvature of the melting interface. The minus sign is due to the $S_{2}$ point to $z$ direction. In numerical tests, $d=\Delta \xi \sqrt{1+\left(\frac{\partial S_{2}}{\partial \xi}\right)^{2}}$, where $\Delta \xi$ is the interval of $\xi$. The interval can be taken as small enough to make $a d<2$.

The basic idea for the curvature-correction is that the energy influence should be equal in the normal direction of the surface (see Figure 3.2). A concave surface tends to reduce the energy influence density while a convex one works exactly in the opposite direction.

Note that $\frac{\partial \theta_{s}}{\partial \zeta}$ in Eq. (3-24) is related to $\left.\frac{\partial \theta_{s}}{\partial n}\right|_{n=0}$ by considering the heat energy fluency crossing the melt surface, which gives

$$
\left.\frac{\partial \theta_{s}}{\partial \zeta}\right|_{n=0}=-C_{c}\left[\frac{\partial \theta_{s}}{\partial n}(0, \tau) \sqrt{1+\left(\frac{\partial S_{2}}{\partial \xi}\right)^{2}}+\frac{\theta_{i}}{N_{\alpha}} \frac{\partial S_{2}}{\partial \tau}\right]
$$

Substituting (3-39) and the definition of $V_{n}$ into (3-24) leads to

$$
\frac{1}{N_{\alpha}}\left(\frac{1}{\operatorname{steN}_{c}}-C_{c} \theta_{i}\right) \frac{\partial S_{2}}{\partial \tau}=C_{c} \frac{\partial \theta_{s}}{\partial n}(0, \tau) \sqrt{1+\left(\frac{\partial S_{2}}{\partial \xi}\right)^{2}}+\left.k \frac{\partial \theta_{l}}{\partial \zeta}\right|_{\zeta=0}
$$




\subsection{Some examples of energy flux rate in solid via heat conduction}

Although the exact solution of temperature and its gradient is explicitly given in the last session, it is possible that it is not found intuitively; thus, examples are provided to show the solutions. The physical parameters are listed in Table 3.1.

Firstly, the temperature profiles at four locations at corresponding melting start time are shown in the Figure 3-3a. They are the established temperature profiles at the very beginning of melting. At the center of the laser beam, the melting preheat time is the shortest and the profile is the narrowest. The farther away from the center, the less the laser beam energy intensity, and more time required for temperature to reach melting point, and the wider the profile. Figure 3-3b shows the temperature profiles generated from the Stefan problem at a specific time, which leads to the same heat flux as in the pure conduct case. Figure 3-3c shows the differences between the established profiles and the generated profiles. Obviously, the difference is non-zero except at the boundaries ( $n=0$ and $n=\infty$ ). Ignoring the difference means the energy stored from pre-melting is missing.

Treating the temperature difference as an existed temperature profile, we can calculate the diffusion and the result is shown in Figure 3-4. It can be seen that the temperature peak value drops very quickly. At the first one $\tau_{m}$, the peak value is only $1 / 3$ of the original value. The temperature at the boundary rises from zero and then declines. The details of temperature change at the boundary is shown in Figure 3-5. The maximum temperature value occurs at $0.23 \tau_{m}$ and the maximum gradient comes even earlier. Because boundary temperature is always lower than the value in the interior (heat always diffuses from high temperature to low), the gradient is always positive. 
To maintain the zero temperature at the boundary, we have to add a negative temperature to cancel out the non-zero value caused by the existing profile. The evolution of changing temperature at the boundary is shown in Figure 3.6. It can be observed that the profile becomes flatter and wider with time.

The exact solution consists of three components. The major component is the solution of the Stefan problem. The other two are the correction of underestimated profile and further correction for maintaining zero temperature at the boundary. The exact solution and its three components are shown in Figure 3.7 for the laser beam center. The solutions are same for other locations because the horizontal axis is normalized with melting time. From Figure 3.7, it is observed that the corrections mainly work in the early stage. After $5 \tau_{m}$, the solution of the Stefan problem is very close to the exact solution. In most applications, the laser pulse duration is much longer than $5 \tau_{m}$, thus, we may use Stefan problem solution as an approximate. This way we may avoid wasting of computation resource on the corrections and assure the computing accuracy.

For time less than $5 \tau_{m}$, the correction is necessary. By simply moving the Stefan solution leftward by a couple of $\tau_{0}$, the misfit can be significantly reduced. Figure 3-8 shows two approximates: one is to shift the Stefan solution by $\tau_{0}$ and is marked by "approximate 1"; and the other is to migrate the Stefan solution with time leftward moving by $3 \tau_{0}$ (including the original $\left.\tau_{0}\right)$. It shows that the migrated Stefan solution by $3 \tau_{0}$ is a better approximate. 
Table 3.1: Physical parameters in the exampled application

\begin{tabular}{|l|c|c|}
\hline initial temperature & $\theta_{i}$ & -0.733 \\
\hline thermal diffusivity ratio & $N_{\alpha}$ & 4.0 \\
\hline ratio of thermal conductivity of liquid over solid & $k$ & 0.412 \\
\hline dimensionless coefficient & $k^{\prime}$ & 0.0161 \\
\hline
\end{tabular}




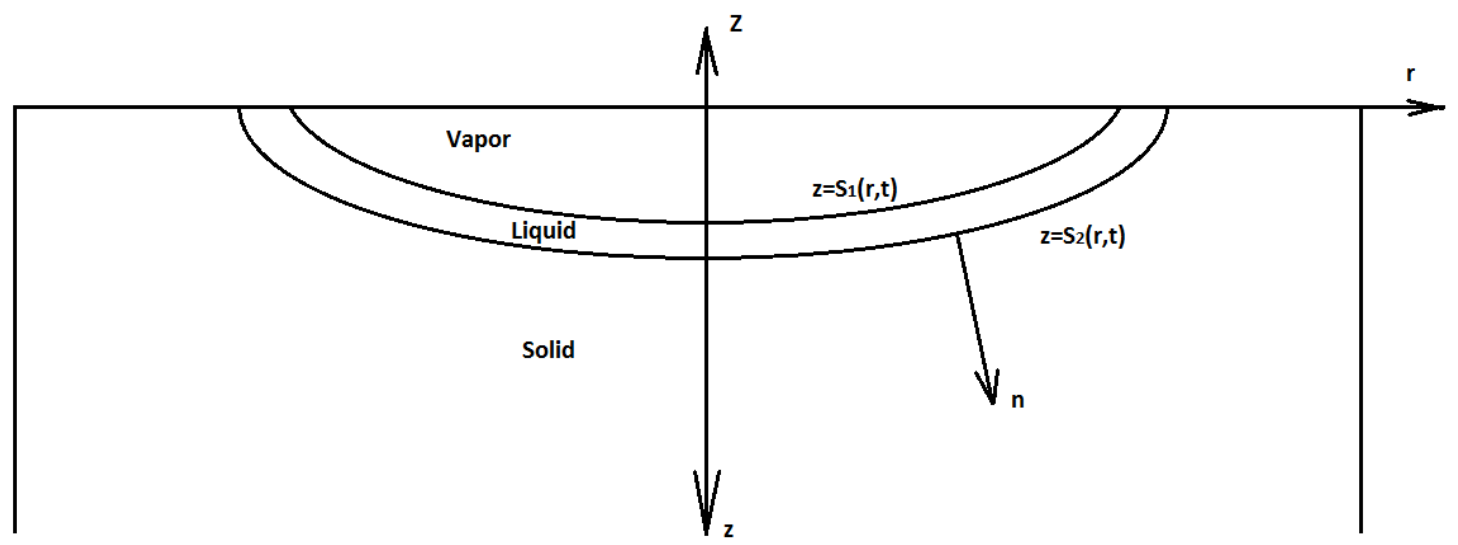

Figure 3.1. Physical model of laser drilling and the coordinate systems used.

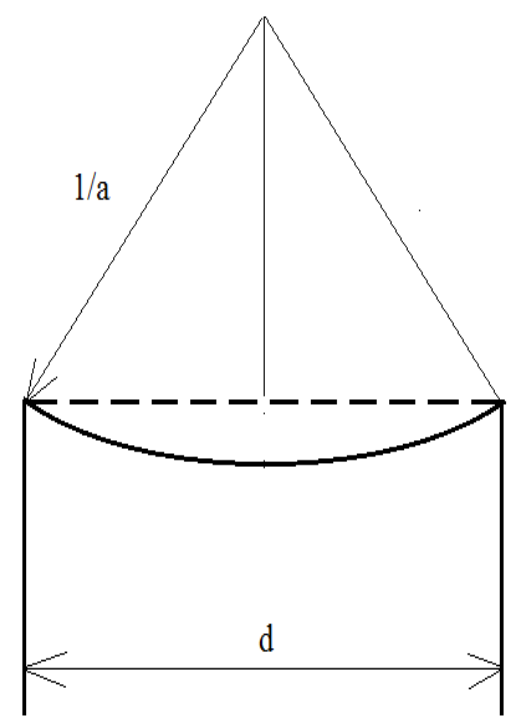

Figure 3.2. Flux change due to the curvature of the solid-melt interface. 


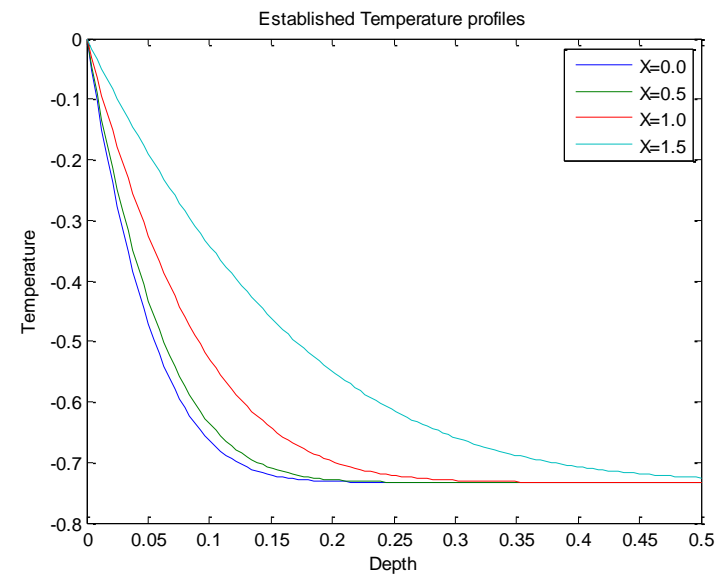

A

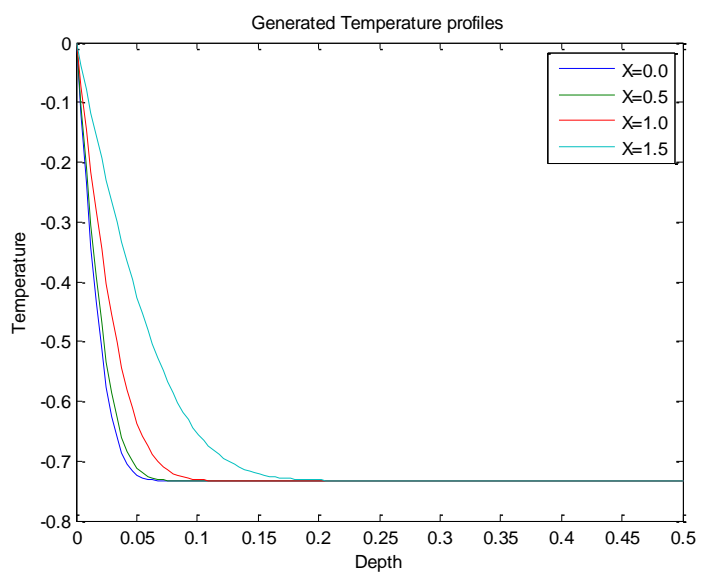

B

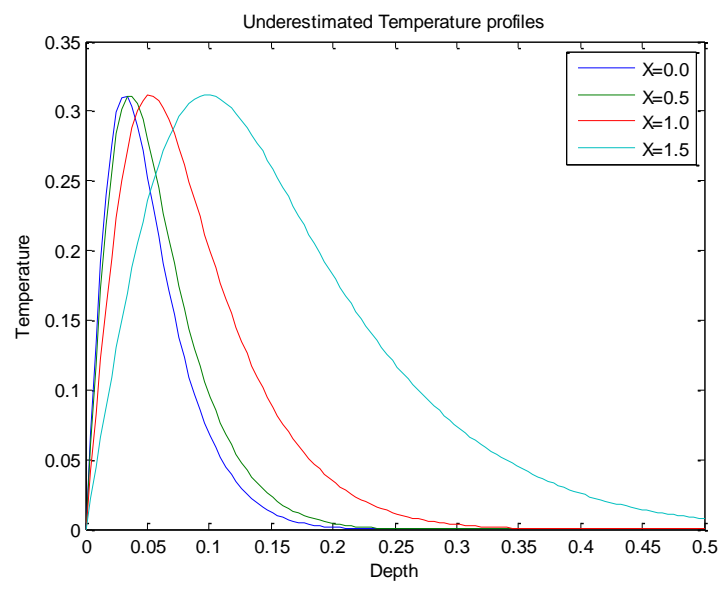

C

Figure 3.3 a) The established temperature at the very beginning of melting; $b$ ) the assumed (generated) temperature profile; and c) the underestimated temperature at the moment of melting starts. 


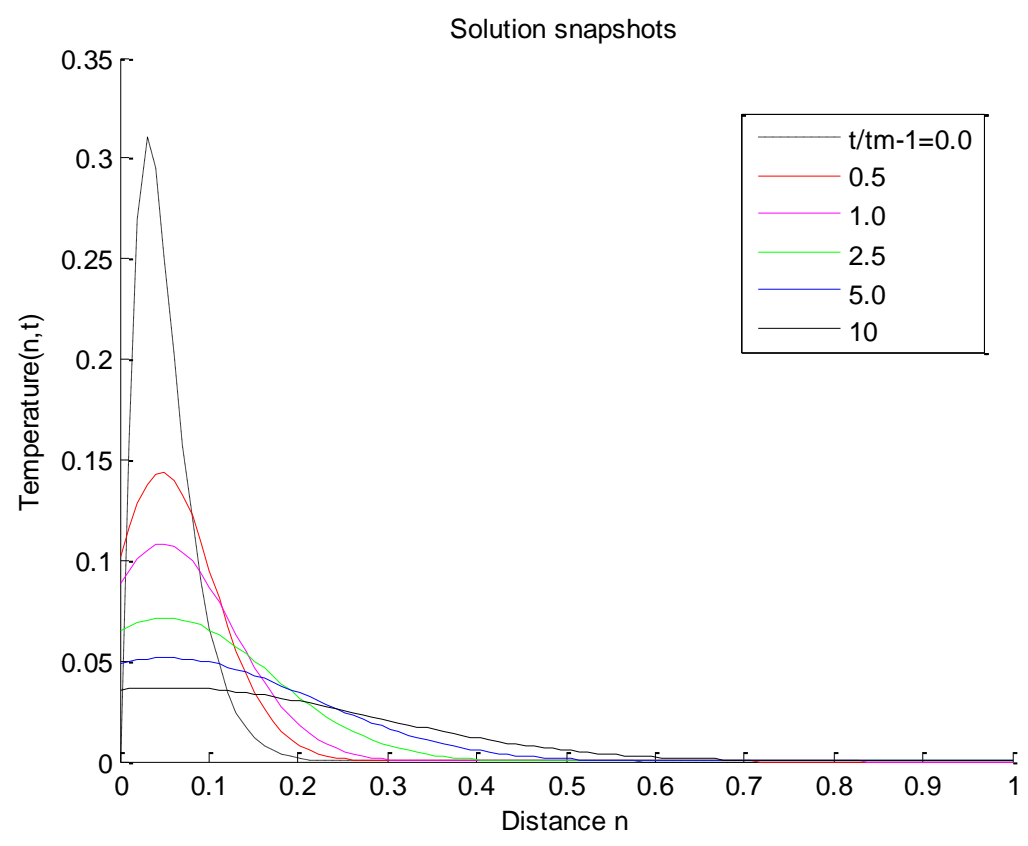

Figure 3.4 Diffusion of the existed temperature profile. 

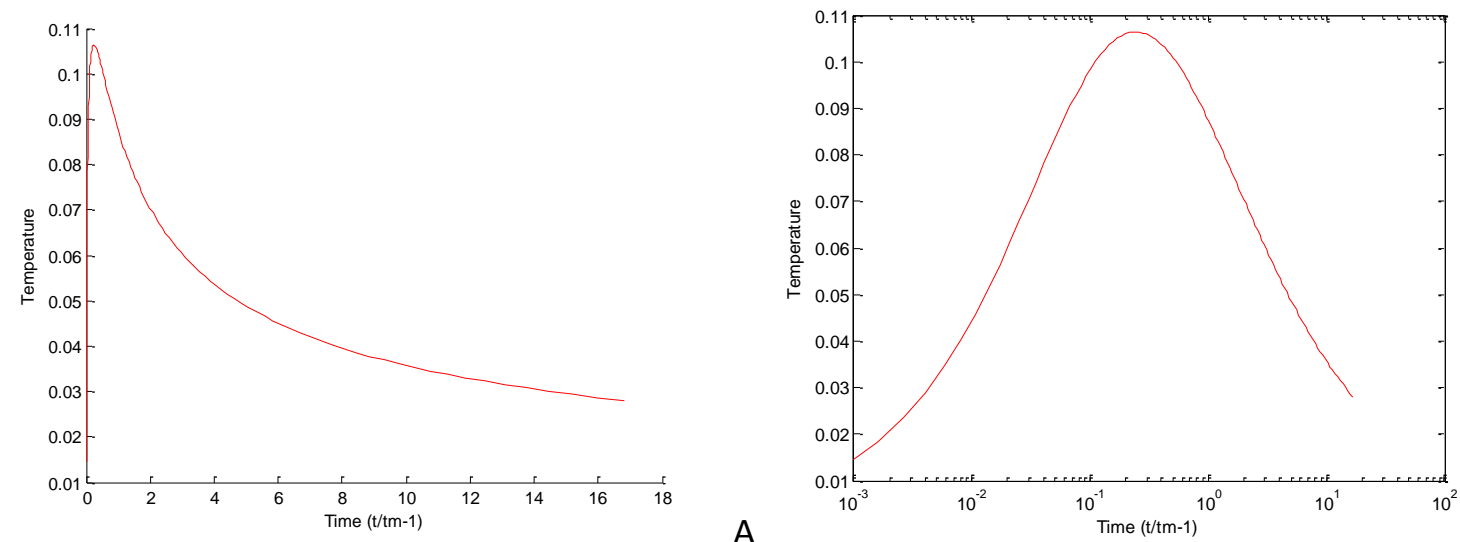

B
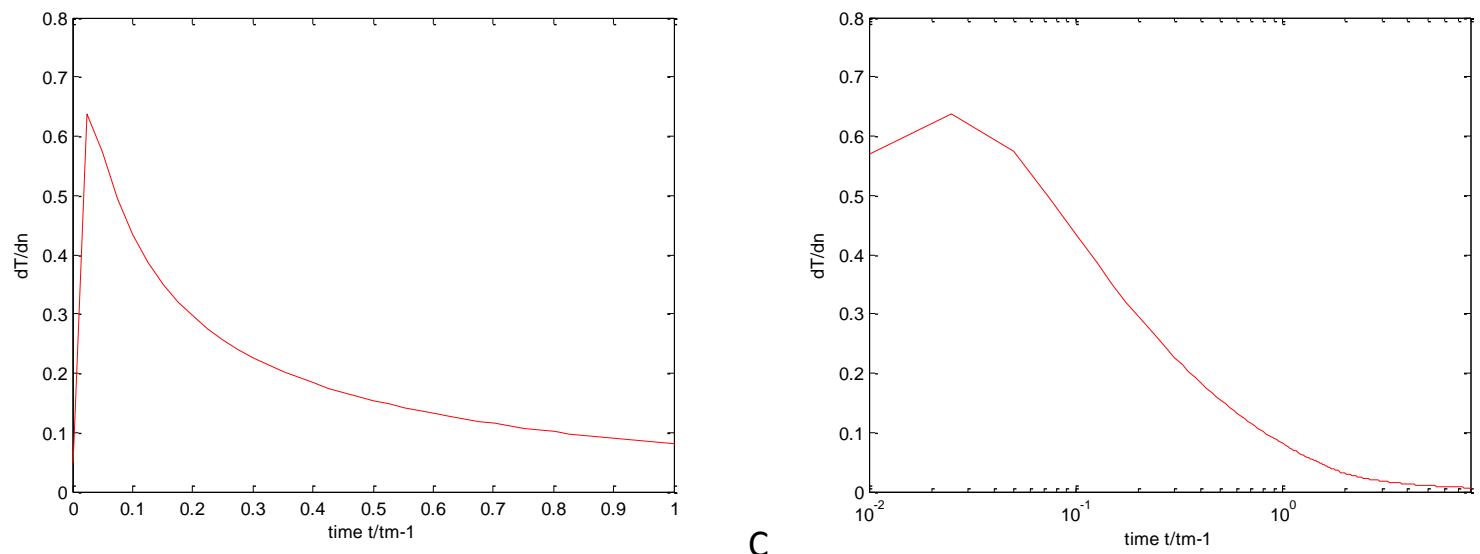

D

Figure 3.5 Temperature history at the melting surface due to the diffusion of the existed temperature profile. A) Drawn on a linear time axis, showing the declining effect with time; B) in logarithm axis of time, showing a rising stage at the very beginning. The temperature gradient history at the solid-liquid boundary C) in linear axis of time; D) in logarithm axis of time 


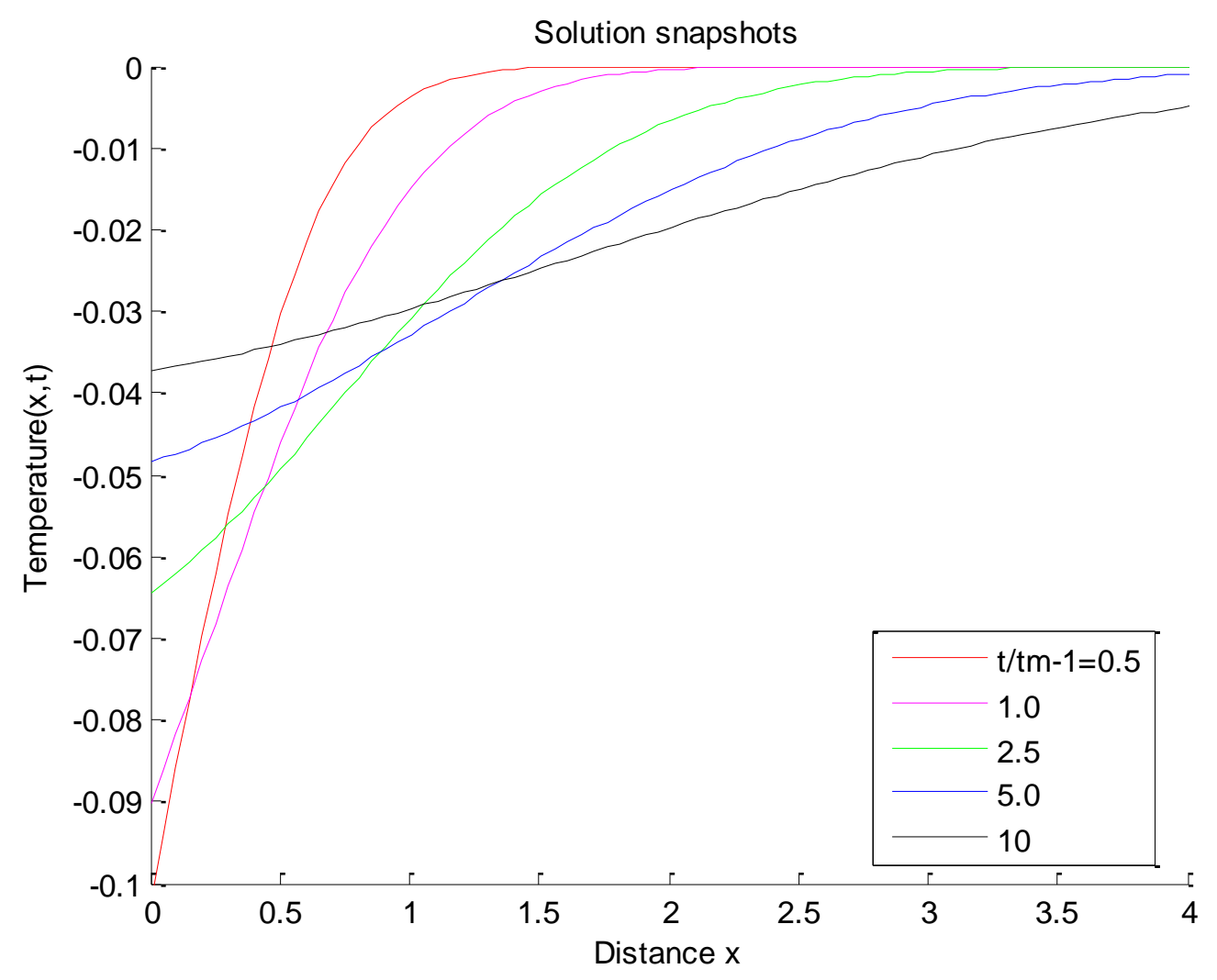

Figure 3.6 Temperature diffusion due to varying boundary condition. 


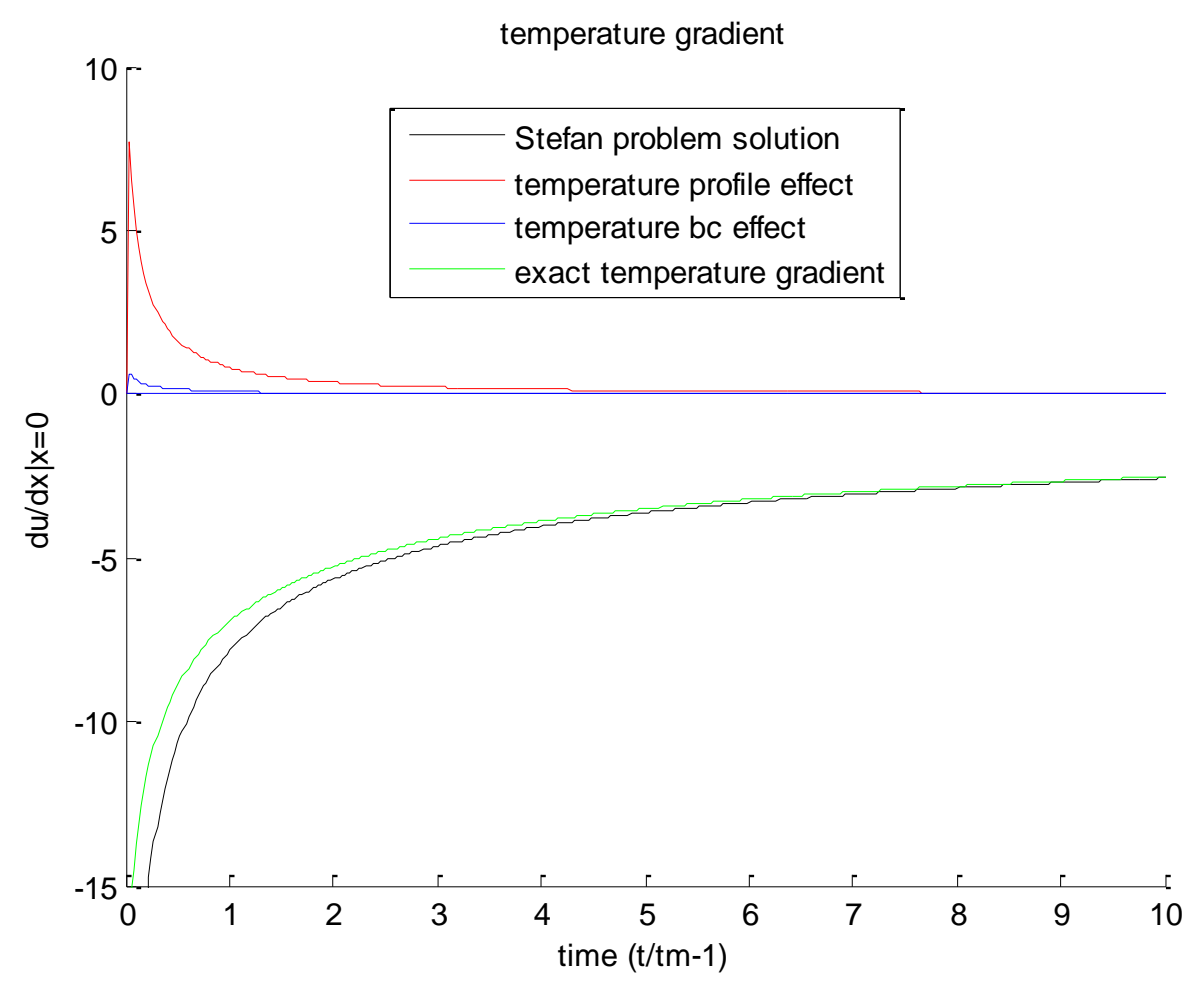

Figure 3.7 Temperature gradient history at the solid-liquid boundary.

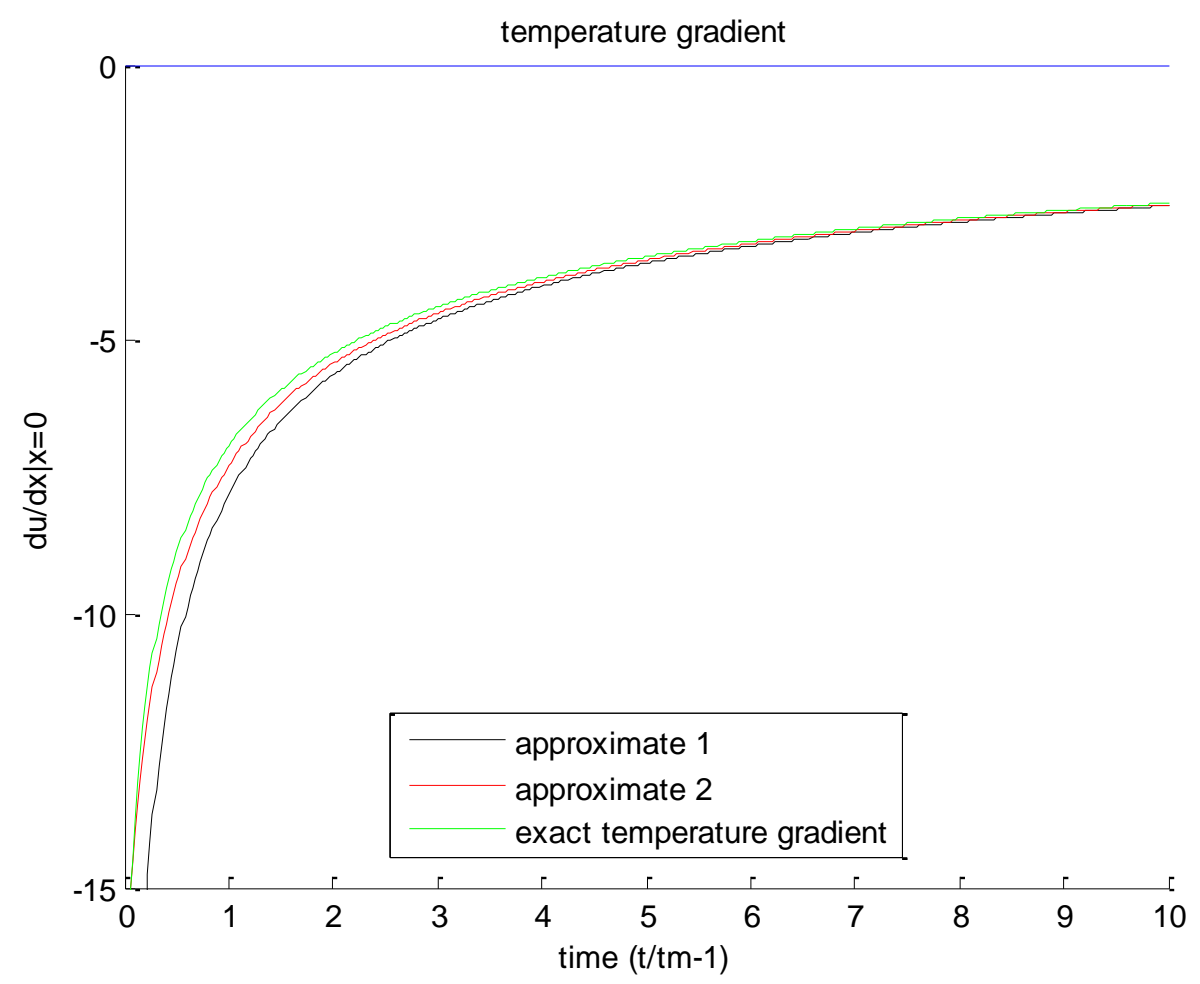

Figure 3.8 Exact solution and approximated solutions at the solid-liquid boundary. 


\section{Chapter 4. Numerical Solutions of the melt flow}

Equations (3-24) and (3-40) cannot be solved without knowing the temperature variation in the melt. The temperature gradient is influenced by the melt flow as shown in the equation of energy conservation (3-3b). Solving the flow field becomes the key point in seeking a more accurate temperature solution, which makes this research unique by comparing it with previous research. In this section, we focus on solving the equation of momentum conservation. Successful applications for this purpose can be found in natural and forced convection near fluid-solid interface by using boundary-layer theory (Faghri, Zhang et al. 2010). Our first idea was to seek the solution by applying boundary layer theory.

\subsection{Boundary layer formulation}

Integrating Eq. (3-1b) with respect to $\zeta$ from 0 to $\delta$, one gets the velocity at the top of the boundary layer:

$$
v_{\delta}=-\int_{0}^{\delta}\left(\frac{\partial u}{\partial \xi}+\frac{u}{\xi}\right) d \zeta
$$

Note that

$$
\int_{0}^{\delta} v \frac{\partial u}{\partial \zeta} d \zeta=-u(\xi, \delta) \int_{0}^{\delta}\left(\frac{\partial u}{\partial \xi}+\frac{u}{\xi}\right) d \zeta+\int_{0}^{\delta} u\left(\frac{\partial u}{\partial \xi}+\frac{u}{\xi}\right) d \zeta
$$

Integrating the momentum equation (3-2b) with respect to $\zeta$ from 0 to $\delta$, results in

$$
\begin{aligned}
& \int_{0}^{\delta} u \frac{\partial u}{\partial \xi} d \zeta-u(\xi, \delta) \int_{0}^{\delta}\left(\frac{\partial u}{\partial \xi}+\frac{u}{\xi}\right) d \zeta+\int_{0}^{\delta} u\left(\frac{\partial u}{\partial \xi}+\frac{u}{\xi}\right) d \zeta=\int_{0}^{\delta} \operatorname{Pr}\left[\frac{\partial^{2} u}{\partial \zeta^{2}}+\frac{1}{\xi} \frac{\partial u}{\partial \xi}-\frac{u}{\xi^{2}}\right] d \zeta+ \\
& \int_{0}^{\delta} U \frac{d U}{d \xi} d \zeta
\end{aligned}
$$


From $-\frac{d p}{d \xi}=U \frac{d U}{d \xi}$, we have $\frac{1}{2} U^{2}+p=$ Constant. At the center of laser beam, $\xi=0, U=$ $0, p=p_{c}$, where $p_{c}$ is the pressure at the beam center, thus, one gets

$$
U=\sqrt{2\left(p_{c}-p(\xi)\right)}
$$

Beneath the free flow, the radial velocity is assumed to be in the form of

$$
u=U\left(2 \frac{\zeta}{\delta}-\frac{\zeta^{2}}{\delta^{2}}\right)
$$

which automatically meets boundary conditions of (3-6) to (3-9).

Substituting Eq. (4-5) and the derivatives into (4-3), one gets

$$
\left(\operatorname{Pr}-\frac{16}{5} \xi U\right) \frac{d \delta}{d \xi}=-\operatorname{Pr}\left(\frac{3 \xi}{\delta}-\frac{\delta}{U} \frac{d U}{d \xi}+\frac{\delta}{\xi}\right)+\frac{3}{4} \xi \delta \frac{d U}{d \xi}+\frac{1}{8} \delta U
$$

This differential equation can be solved using the Runge-Kutta method (Burden and Faires 2012) with a certain boundary condition. With zero free flow velocity at the center, it seems that any value of the boundary layer thickness will work. This uncertainty forces our attention to the second node. By assuming $\frac{d \delta}{d \xi}=0$ at the beam center, which reflects the axisymmetric condition and approximating $\frac{d U}{d \xi}$ with $\frac{U}{\xi}$, we get $\delta=\sqrt{\frac{24 \xi P r}{7 U}}$ from Eq. (4-6). Note, this formula holds approximately only at the second node. Using this boundary value, the succeeding values will be solved with the Runge-Kutta method being applied to Eq. (4-6).

The boundary layer flow velocity profile may also be assumed to be a cubic function as follows

$$
u=U\left(\frac{3}{2} \frac{\zeta}{\delta}-\frac{1}{2} \frac{\zeta^{3}}{\delta^{3}}\right)
$$


which automatically meets the boundary conditions in (3-6) to (3-9).

Integrating the continuity equation, one gets

$$
v=-\int_{0}^{\zeta}\left(\frac{\partial u}{\partial \xi}+\frac{u}{\xi}\right) d \zeta=-\frac{\delta}{8}\left(\frac{d U}{d \xi}+\frac{U}{\xi}\right)\left[6\left(\frac{\zeta}{\delta}\right)^{2}-\left(\frac{\zeta}{\delta}\right)^{4}\right]+\frac{3}{8} U \frac{d \delta}{d \xi}\left[2\left(\frac{\zeta}{\delta}\right)^{2}-\left(\frac{\zeta}{\delta}\right)^{4}\right]
$$

Substituting the above and Eq. (4-7) into Eq. (4-3) one gets

$$
(105 \operatorname{Pr}-39 \xi U) \frac{d \delta}{d \xi}=-35 \operatorname{Pr}\left(\frac{12 \xi}{\delta}-5 \frac{\delta}{U} \frac{d U}{d \xi}+5 \frac{\delta}{\xi}\right)+39 \delta U+183 \xi \delta \frac{d U}{d \xi}
$$

which can be solved by using the boundary condition $\delta=\sqrt{\frac{70 \operatorname{Pr\xi }}{37 U}}$ at the second node similar to the reasoning discussed in the previous paragraph for Eq. (4-6).

The free flow $U$ could lead to the melt layer thickness change. The mass conservation requires

$$
\frac{\partial U}{\partial \xi}+\frac{\partial V}{\partial \zeta}+\frac{U}{\xi}=0
$$

Integrated Eq. (4-10) over $\zeta$ from $\zeta=\delta$ to $\zeta=g$, one has

$$
V=-\left(\frac{\partial U}{\partial \xi}+\frac{U}{\xi}\right)(g-\delta)
$$

The vertical velocity at the surface of the boundary layer is

$$
v_{\delta}=-\int_{0}^{\delta}\left(\frac{\partial u}{\partial \xi}+\frac{u}{\xi}\right) d \zeta
$$

For the profile represented in Eq. (4-5), $v_{\delta}=-\frac{2}{3} \delta\left(\frac{\partial U}{\partial \xi}+\frac{U}{\xi}\right)$

For the profile represented in Eq. (4-7), $v_{\delta}=-\frac{5}{8} \delta\left(\frac{\partial U}{\partial \xi}+\frac{U}{\xi}\right)$ 
The melt layer thickness varies due to melting rate, evaporating rate, and the flow-related changes as shown below:

$$
\frac{\partial g}{\partial \tau}=\frac{\partial S_{2}}{\partial \tau}-\frac{\partial S_{1}}{\partial \tau}+V+v_{\delta}
$$

which can be used to update the melt layer thickness, i.e.

$$
g=g_{0}+\left(\frac{\partial S_{2}}{\partial \tau}-\frac{\partial S_{1}}{\partial \tau}+V+v_{\delta}\right) \Delta \tau
$$

where $g_{0}$ denotes the melt thickness at the previous time step and $g$ at current step. $\Delta \tau$ is the time interval.

Note that $\frac{\partial S_{1}}{\partial \tau}$ represents the melt surface change due to vaporization only. Considering vertical displacement due to flow, we get the net melt-vapor interface

$$
S_{1 n e t}=S_{2}-g
$$

The space between the initial solid surface and the net melt surface will be counted as the material removed by the laser beam because the melt will be recast once the heating process ends.

\subsection{Integration method formulation}

Near the laser beam center, the boundary layer is thin. Far from the beam center, the boundary layer increases and the free flow layer may disappear. In this case, because boundary conditions (3-6) and (3-7) no longer remain valid, we return to Eq. (3-2a). We now seek an integral solution. Eq. (4-3) is rewritten as

$$
\int_{0}^{g} u \frac{\partial u}{\partial \xi} d \zeta-u(\xi, g) \int_{0}^{g}\left(\frac{\partial u}{\partial \xi}+\frac{u}{\xi}\right) d \zeta+\int_{0}^{g} u\left(\frac{\partial u}{\partial \xi}+\frac{u}{\xi}\right) d \zeta=\int_{0}^{g} \operatorname{Pr}\left(\frac{\partial^{2} u}{\partial \zeta^{2}}+\frac{1}{\xi} \frac{\partial u}{\partial \xi}-\frac{u}{\xi^{2}}\right) d \zeta-g \frac{d p}{d \xi}
$$


The radial velocity must have experienced a rising course since it is zero at the laser beam center. However, the radial velocity profile cannot be $u=A \xi \zeta$, because this function will lead to $\frac{d p}{d \xi}=0$, which means the recoil pressure is constant in the melt zone. We cannot assume a profile like $u=A \zeta \xi^{2}$ or any power index larger than 1 because a free flow cannot be that way with respect to the distance. If the radial velocity is $u=A \zeta \xi^{1 / m}$ where $m>1$ and $A$ is a coefficient independent to either coordinate, then $v=-\int_{0}^{\zeta}\left(\frac{\partial u}{\partial \xi}+\frac{u}{\xi}\right) d \zeta=-\frac{1+m}{2 m} A \xi^{\frac{1}{m}-1} \zeta^{2}$. This shape function predicts that the melt surface falls in the entire melt zone. Substituting $u=A \zeta \xi^{1 / m}$ into Eq. (4-16), and after algebraic operations, one obtains

$$
A^{2} g^{2} \xi^{\frac{2}{m}+1}-3 \operatorname{Pr} A g \xi^{\frac{1}{m}}-\frac{6 m}{m-1} \xi^{2} \frac{d p}{d \xi}=0
$$

Although the coefficient $A$ can be solved locally, a potential problem is that $A$ may vary with location, which contradicts our definition of $A$. Instead, we seek a constant $A$ that makes the least square of the residuals, i.e.

$$
f(A)=\int_{0}^{\infty}\left(A^{2} g^{2} \xi^{\frac{2}{m}+1}-3 \operatorname{Pr} A g \xi^{\frac{1}{m}}-\frac{6 m}{m-1} \xi^{2} \frac{d p}{d \xi}\right)^{2} d \xi
$$

To find $A$ that makes $f(A)$ minimum, let $\frac{d f(A)}{d A}=0$, and one gets

$$
W(A)=Q_{1} A^{3}-Q_{2} A^{2}+Q_{3} A-Q_{4}=0
$$

where 


$$
\left\{\begin{array}{c}
Q_{1}=\int_{0}^{\infty} 2 g^{4} \xi^{\frac{4}{m}+2} d \xi \\
Q_{2}=9 \operatorname{Pr} \int_{0}^{\infty} g^{3} \xi^{\frac{3}{m}+1} d \xi \\
Q_{3}=\int_{0}^{\infty}\left(-\frac{12 m}{m-1} \xi^{3} \frac{d p}{d \xi}+9 \operatorname{Pr}^{2}\right) g^{2} \xi^{\frac{2}{m}} d \xi \\
Q_{4}=-\frac{18 m}{m-1} \operatorname{Pr} \int_{0}^{\infty} g \xi^{\frac{1}{m}+2} \frac{d p}{d \xi} d \xi
\end{array}\right.
$$

It is clear that $W(0)=-Q_{4}<0$ and $W(+\infty)>0$, and two stationary points make

$$
\frac{d W}{d A}=3 Q_{1} A^{2}-2 Q_{2} A+Q_{3}=0
$$

The two stationary points are $A_{1}=\frac{\left(Q_{2}-\sqrt{Q_{2}{ }^{2}-3 Q_{1} Q_{3}}\right)}{3 Q_{1}}>0$, and $A_{2}=\frac{\left(Q_{2}+\sqrt{Q_{2}{ }^{2}-3 Q_{1} Q_{3}}\right)}{3 Q_{1}} \geq A_{1}$.

If $W\left(A_{1}\right)<0$, there is only one real root that exists in domain $\left(A_{2},+\infty\right)$. If $W\left(A_{1}\right) \geq 0$, there are three real roots, but only the root in domain $\left(0, A_{1}\right]$ is meaningful in physics and mathematics. The Newton-Raphson method is used for solving the coefficient $A$.

Since there is no knowledge about the value of the index $m$, we take $m=1.5,2,3,4,5$ for numerical tests. Through comparing numerical prediction with experimental data, we may find the best value of $m$.

Another possibility is that the radial velocity drops after a peak value somewhere in the melt zone. In this case, a smooth shape function $u=A \xi \zeta \exp \left(-\xi / \xi_{p}\right)$ is assumed, where $\xi_{p}$ is the radius at which the radial velocity is maximum.

$$
v=-\int_{0}^{\zeta}\left(\frac{\partial u}{\partial \xi}+\frac{u}{\xi}\right) d \zeta=-\frac{A \zeta^{2}}{2}\left(2-\frac{\xi}{\xi_{p}}\right) \exp \left(-\frac{\xi}{\xi_{p}}\right)=-\zeta\left(\frac{1}{\xi}-\frac{1}{2 \xi_{p}}\right) u
$$

This shape function predicts the melt surface subsides in the domain $\left(0,2 \xi_{p}\right)$ whereas it rises beyond $2 \xi_{p}$. Since there is no knowledge about $\xi_{p}$, we may try $\xi_{p}=0.2,0.4,0.6,0.8,1.0,1.5$ and 
see which value leads to the best fit to the experimental data. Substituting $u=A \xi \zeta \exp \left(-\xi / \xi_{p}\right)$ into the momentum conservation Eq.(4-16) gives

$$
A^{2} g^{2} \xi^{2}-3 \operatorname{Pr} A g \exp \left(\frac{\xi}{\xi_{p}}\right)-6 \xi_{p} \frac{d p}{d \xi} \exp \left(\frac{2 \xi}{\xi_{p}}\right)=0
$$

Similar to the previous analysis, we seek a constant coefficient $A$ in terms of least square of the residuals, and we get same form of Eq. (50) with

$$
\left\{\begin{array}{c}
Q_{1}=\int_{0}^{\infty} 2 g^{4} \xi^{4} d \xi \\
Q_{2}=9 \operatorname{Pr} \int_{0}^{\infty} g^{3} \xi^{2} \exp \left(\frac{\xi}{\xi_{p}}\right) d \xi \\
Q_{3}=\int_{0}^{\infty}\left(9 \operatorname{Pr}^{2}-12 \xi_{p} \xi^{2} \frac{d p}{d \xi}\right) g^{2} \exp \left(\frac{2 \xi}{\xi_{p}}\right) d \xi \\
Q_{4}=-18 \operatorname{Pr} \xi_{p} \int_{0}^{\infty} g \frac{d p}{d \xi} \exp \left(\frac{3 \xi}{\xi_{p}}\right) d \xi
\end{array}\right.
$$

\subsection{Solution of the temperature field}

Now we work on the energy equation Eq. (3-3b). The following temperature profile is assumed in the liquid (melt)

$$
\theta_{l}=\left(\frac{\theta_{s a t}}{g}-b_{2} g\right) \zeta+b_{2} \zeta^{2}
$$

which satisfies the boundary conditions in (3-10) and (3-11). The vertical gradient is

$$
\frac{\partial \theta_{l}}{\partial \zeta}=\frac{\theta_{\text {sat }}}{g}-b_{2} g+2 b_{2} \zeta
$$

At the solid-melt interface, $\left.\frac{\partial \theta_{l}}{\partial \zeta}\right|_{\zeta=0}=\frac{\theta_{s a t}}{g}-b_{2} g$; at the melt-vapor interface $\left.\frac{\partial \theta_{l}}{\partial \zeta}\right|_{\zeta=g}=$ $\frac{\theta_{s a t}}{g}+b_{2} g$ 
Now we solve Eq. (3-3b) using the assumed temperature profile and solved flow velocity. Integrating Eq. (3-3b) with respect to $\zeta$ from 0 to $g$, one gets

$$
\frac{\partial}{\partial \xi} \int_{0}^{g} u \theta_{l} d \zeta+\int_{0}^{g} \theta_{l} \frac{u}{\xi} d \zeta+v(\xi, g) \theta_{s a t}=2 b_{2} g
$$

Substituting Eq. (4-25) into Eq. (3-23), one gets

$$
\begin{array}{r}
\left\{1+\Lambda\left[\theta_{\text {sat }}\left(1-\theta_{m}\right)+\theta_{m}\right]\right\} \exp \left[H_{l v}\left(1-\frac{1}{\theta_{\text {sat }}\left(1-\theta_{m}\right)+\theta_{m}}\right)\right]= \\
I^{\prime} \sqrt{\theta_{\text {sat }}\left(1-\theta_{m}\right)+\theta_{m}}\left[\exp \left(-\xi^{2}\right)-k^{\prime}\left(\frac{\theta_{s a t}}{g}+b_{2} g\right)\right]
\end{array}
$$

Let $\theta_{\text {sat }}\left(1-\theta_{m}\right)+\theta_{m}=\varphi$, one converts (4-28) into

$$
(1+\Lambda \varphi) \exp \left[H_{l v}\left(1-\frac{1}{\varphi}\right)\right]=I^{\prime} \sqrt{\varphi}\left[\exp \left(-\xi^{2}\right)-k^{\prime}\left(\frac{\varphi-\theta_{m}}{g\left(1-\theta_{m}\right)}+b_{2} g\right)\right]
$$

After algebraic operations, equation (4-29) can be rewritten as

$$
A(1+\Lambda \varphi) \exp \left(-\frac{B}{\varphi}\right)+(C \varphi-D) \sqrt{\varphi}=0
$$

where $A=\frac{1}{I^{\prime}} \exp \left(H_{l v}\right), B=H_{l v}, C=\frac{k^{\prime}}{g} \frac{1}{1-\theta_{m}}, D=\exp \left(-\xi^{2}\right)-k^{\prime}\left[b_{2} g-\frac{\theta_{m}}{g\left(1-\theta_{m}\right)}\right]$.

In the pure conduction model, $b_{2}=0$, and the temperature can be solved directly through the Newton-Raphson method. In the convection model, $b_{2}$ is a variable and is constrained by the energy balance equation like the one expressed in (4-27). A convenient way is to take the value of $b_{2}$ obtained at the last step as an approximate to calculate $\theta_{\text {sat }}$ if the time interval is sufficiently small. Once $\theta_{\text {sat }}$ is solved in (4-30), the value of $b_{2}$ is updated in (4-27) by using current flow velocity and temperature. 


\section{Chapter 5. Results and discussion}

\subsection{Comparison of the model predicts with experimental data}

The case of pure conduction, and then conduction-flow cases, were simulated and compared with experimental data. The thermophysical properties used are listed in Table 5.1. The laser intensity at the center varies from $5.3 \times 10^{10} \mathrm{~W} \mathrm{~m}^{-2}$ to $14.3 \times 10^{10} \mathrm{~W} \mathrm{~m}^{-2}$ in experiments (Zhang and Faghri 1999), and the range was explored from $2.5 \times 10^{10} \mathrm{~W} \mathrm{~m}^{-2}$ to $16.0 \times 10^{10} \mathrm{~W} \mathrm{~m}{ }^{-2}$ in the numerical modeling. Similar to Zhang and Faghri (Zhang and Faghri 1999), the effect of absorptivity of the target material is ignored. The experimental material removal rate was obtained by scaling micrographs of single shot drilled holes for pulse duration of $700 \mu \mathrm{s}$ and laser radius of $0.254 \mathrm{~mm}$ at the Pratt and Whitney drilling facility at North Haven, CT. The same laser pulse duration, and the same beam intensity profile were taken in the current model as that described in Zhang and Faghri (Zhang and Faghri 1999). The results are shown in Figure 5.1. The time step used in the simulation is mainly $1.0 \times 10^{-7}$. Other time intervals like $5.0 \times 10^{-8}, 1.0 \times 10^{-8}$ were also tested, and they led to a convergent solution. The spatial resolution was 0.0125 . Running a case typically takes about 5 minutes. It can be noticed that the predicted material removal rates increase with the laser beam intensity, generally consistent with the experimental data. It is also noticeable that the predicted results from the model without convection were very close to those predicted from models where melt flow was fully considered. The rising radial velocity models generally predicted a slightly higher removal rate than the rising-fall models. All models with melt flow being considered produced results very close to each other within $2 \%$ difference for all cases with rising profiles and rising-fall profiles. 


\subsection{Drill hole evolution}

Figure 5.2 shows the drilled hole profile history predicted from a rising-fall flow model where $\xi_{b}=1$ and laser beam center intensity $7.5 \times 10^{10}\left[\mathrm{~W} \mathrm{~m}^{-2}\right]$. It was observed that the hole developed much faster in the drill direction than in the lateral direction. These profiles are similar to each other and are similar to the profiles predicted by Zhang and Faghri (Zhang and Faghri 1999). It is observed that the thickness of the melt layer increases in radial direction and the thickest melt layer occurs near the edge of the melt zone where the vaporization starts to become negligible. It is also observed that the vertical drilling rate is almost constant.

\subsection{Vapor pressure and temperature}

This model is capable of predicting the temperature of the vapor or the temperature at the top of the melt. Here, we show the pressure and temperature profiles at the final stage of the drilling predicted from rising-fall models in Figure 5.3 and Figure 5.4. It is observed from Figure 5.3 that the higher the laser beam intensity, the larger the vapor recoil pressure. The pressure inside the melt zone is not homogeneous, but like Gaussian curves; it drops steeply between $\xi=0.5 \sim 1.0$. Pressure at $\xi=0.5$ is about $80 \%$ of the value at the laser beam center while it drops to one third at $\xi=1.0$. Figure 5.4 shows the temperature profiles in the corresponding models. It is observed that the temperature rises as the laser intensity increases. For all tested cases, the temperature dropped in the radial direction, but quite gradually in the majority of the melt zone, which was much different from the pressure profile. The temperature at $\xi=1.5$ was about $70 \%$ of the value at the center; it had a steep slope at the edge of the vapor zone.

Our modeling results also showed that the temperature and pressure are quite stable during laser beam impulse except for rapid rising at the very beginning. This pattern is confirmed in both 
rising profile models and rising-fall profile models (as shown in Figure 5.5. and Figure 5.6). The peak flow velocity is predicted to experience a rapid drop at the beginning and attain a quite stable stage later from the rising models (Figure 5.5A). It is slightly different from that which was predicted from models of rising-fall models with parameter $\xi_{p}<0.7$, in which the flow gradually slows down with time (Figure 5.6A). Models with parameter $\xi_{p}>0.8$ generated similar results as that shown in Figure 5.5. We also noticed that the solution was sometimes not available for models with a small value of $\xi_{p}(0.2$ or 0.4$)$.

\subsection{The contribution of melt flow}

The effect of the melt flow on material removal depends on many factors. One factor is the Prattle number. Figure 5.7 shows the melting rate, vaporization rate, and melt flow velocity in a vertical direction predicted from a model of parameters list in Table 5.1. These rates are shown at two locations: one location is near the laser beam center $\xi=0.0125$ and another is at $\xi=1$. It was observed that the melting rate experienced a rapid drop as the vaporization rate rose and reached an equilibrium rather quickly. The melt flow velocity was very small, about 3-4 orders of magnitude less than the vaporization rate. Models of other profiles led to similar results. This may justify the previous model by Zhang and Faghri (Zhang and Faghri 1999) where the flow was negligible in the super alloy they studied. A recent study showed that natural convection does not play a significant role in melt transport and melt pool geometry (Kar and Rath 2014) using a completely different approach. However, if the material is of different parameters, the melt flow might contribute to the material removal differently. To illustrate this point, we conducted a test in which all other parameters were the same as that in the numerical test shown in Figure 5.7 except

for the Prattle number. The results of the test with Prattle number 142 are shown in Figure 5.8. It 
is observed that melt flow was able to cause about $10 \%$ of the material removal in this assumed case.

\subsection{Discussions}

The boundary layer model was developed in this paper, but we are not presenting the results at this time. The reason is that the boundary layer assumption was found to be applicable very close to the laser beam center where the melt flows slowly. In the majority of the melt zone, the free flow zone did not exist. Another problem was that the beam center was calculated based on a pure conduction model (the radial velocity is zero, but the vertical velocity is hard to estimate), while the adjacent node was based on conduction-flow which led to a differential change in the solid-melt interface. The most debatable issue is most likely the boundary condition. It is unavailable at the beam center and is very roughly estimated at the node next to the center. Even so, we still tried to evaluate the melt flow using boundary layer theory near the center and exploiting the integral method beyond the last node at which the boundary layer theory is applicable, with assumed velocity profiles following falling trend. The results were similar to that shown in previous figures. Again, the reason is because the melt flows very slowly compared with the evaporation rate.

We attempted to find the flow pattern by comparing experimental data with models of various radial velocity profiles. This mission is not completed in the cases of very slow flow as in the case of the super alloy studied here. Later experiments may provide better data to assist with flow pattern identification. 


\subsection{Summary for the New Laser Drilling Model}

The novelty of this research is that the melt flow in laser drilling is explicitly included in the equation of momentum conservation. By applying the non-slip boundary condition at the solidliquid interface, the solution of melt flow was developed by using the boundary layer theory and integral method. An integral solution for the temperature field was also obtained. In addition, exact solutions for heat conduction were developed. The solution of the Stefan problem is the principal component in the exact solution and can be used as an approximate solution for better computational efficiency. The dependence of saturation temperature on the vapor pressure was taken into account by using the Clausius-Clapeyron equation. As compared with previous models, the model developed in this study is more realistic. Applying the new model to a super alloy, we showed that melt flow can be ignored in some cases. The solutions derived here will be further applied to more general cases to evaluate the role of melt flow and vaporization on laser drilling profile evolution and to study the solid material removal efficiency. 
Table 5.1. Thermophysical properties of test material.

\begin{tabular}{|c|l|c|}
\hline Latent heat of melting & $h_{l s}$ & $2.31 \times 10^{4}\left[\mathrm{~J} \mathrm{~kg}^{-1}\right]$ \\
\hline Latent heat of vaporization & $h_{l v}$ & $6.444 \times 10^{6}\left[\mathrm{~J} \mathrm{~kg}^{-1}\right]$ \\
\hline Density of melt & $\rho$ & $8.4 \times 10^{3}\left[\mathrm{kgm}^{-3}\right]$ \\
\hline Vapor molar mass & $\mathrm{M}$ & $0.076\left[\mathrm{~kg} \mathrm{~mol}^{-1}\right]$ \\
\hline Initial temperature & $T_{i}$ & $293.15 \mathrm{~K}$ \\
\hline Melting temperature & $T_{m}$ & $1510^{\circ} \mathrm{C}$ \\
\hline Standard Saturation temperature & $T_{s a t 0}$ & $3170^{\circ} \mathrm{C}$ \\
\hline Standard Saturation pressure & $p_{0}$ & $1.01325 \times 10^{5}\left[\mathrm{Nm}^{-2}\right]$ \\
\hline Thermal conductivity of the liquid & $k_{l}$ & $21.70\left[\mathrm{Wm}^{-1} \mathrm{~K}^{-1}\right]$ \\
\hline Thermal conductivity of the solid & $k_{s}$ & $52.72\left[\mathrm{Wm}^{-1} \mathrm{~K}^{-1}\right]$ \\
\hline Specific heat of the liquid & $c_{p l}$ & $625\left[\mathrm{~J} \mathrm{~kg}^{-1} \mathrm{~K}^{-1}\right]$ \\
\hline Specific heat of the solid & $c_{p s}$ & $380\left[\mathrm{~J} \mathrm{~kg}^{-1} \mathrm{~K}^{-1}\right]$ \\
\hline Radius of laser beam & $\mathrm{R}$ & $0.254 \times 10^{-3}[\mathrm{~m}]$ \\
\hline Prattle Number & Pr & 0.142 \\
\hline
\end{tabular}




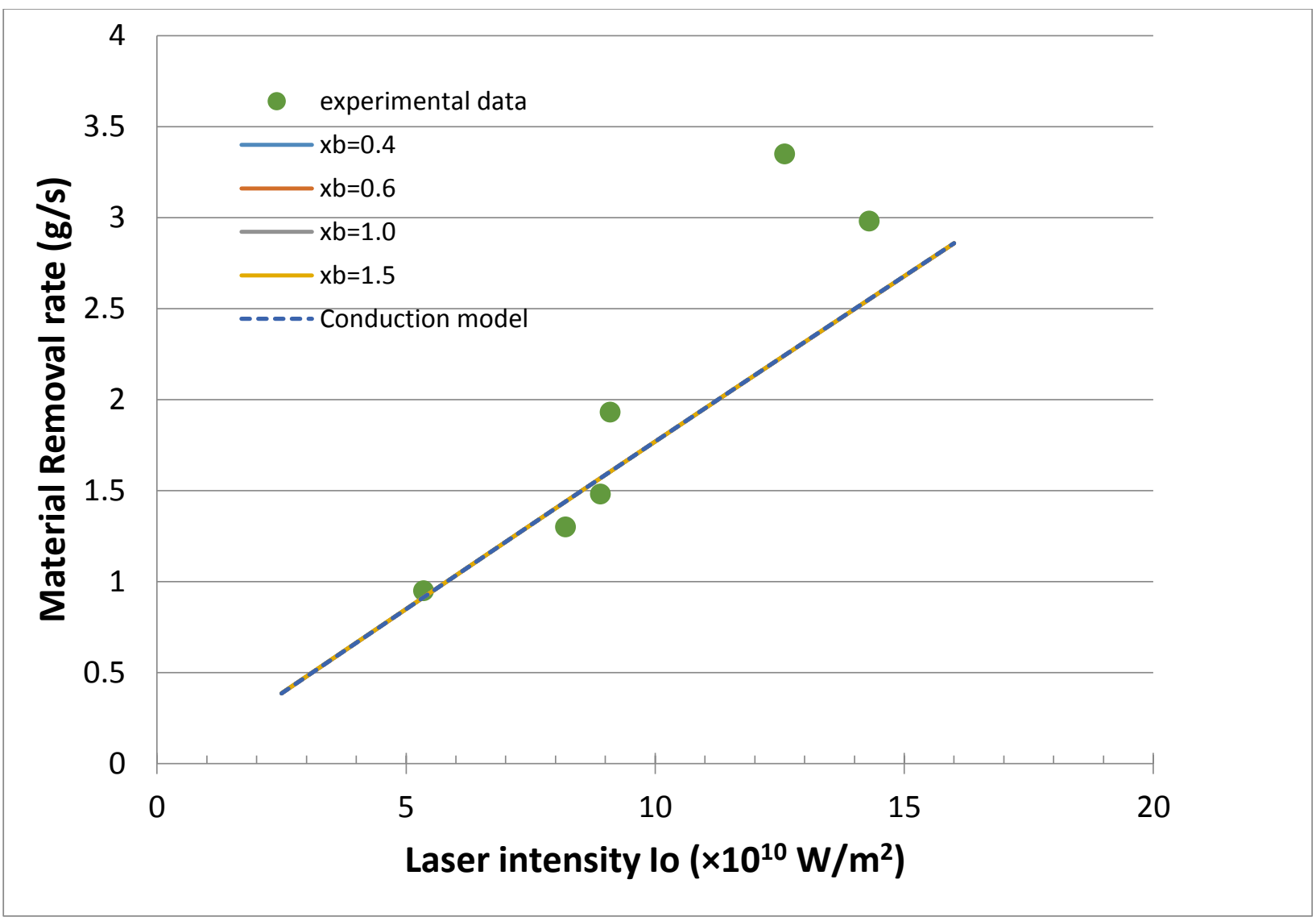

Figure 5.1 Comparison of predicted and experimental material removal rates using models of rising-fall profiles. 


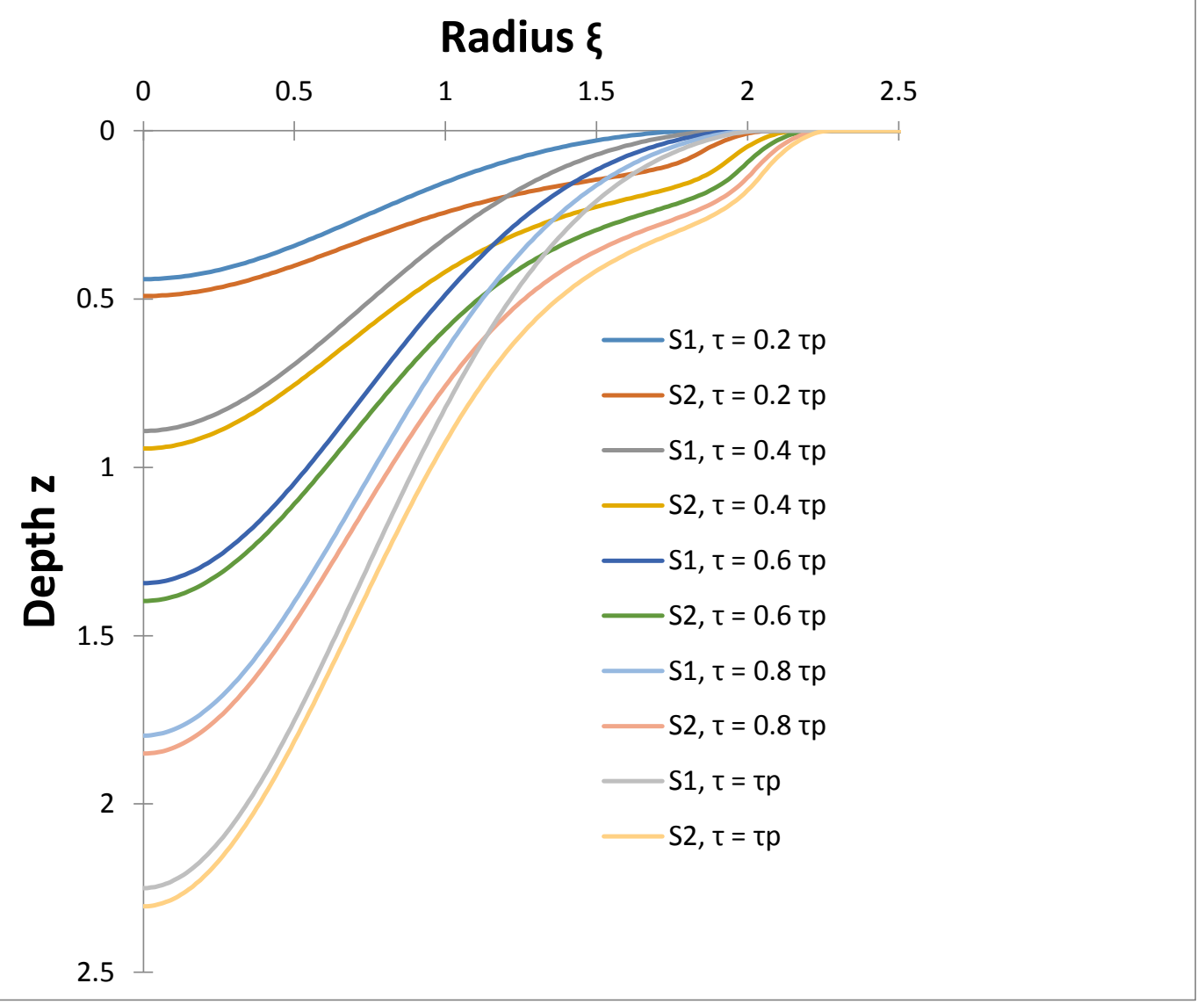

Figure 5.2. Drill hole profiles evolution predicted from a model assuming rising-fall radial velocity profile with peak velocity at $\xi_{p}=1$. 


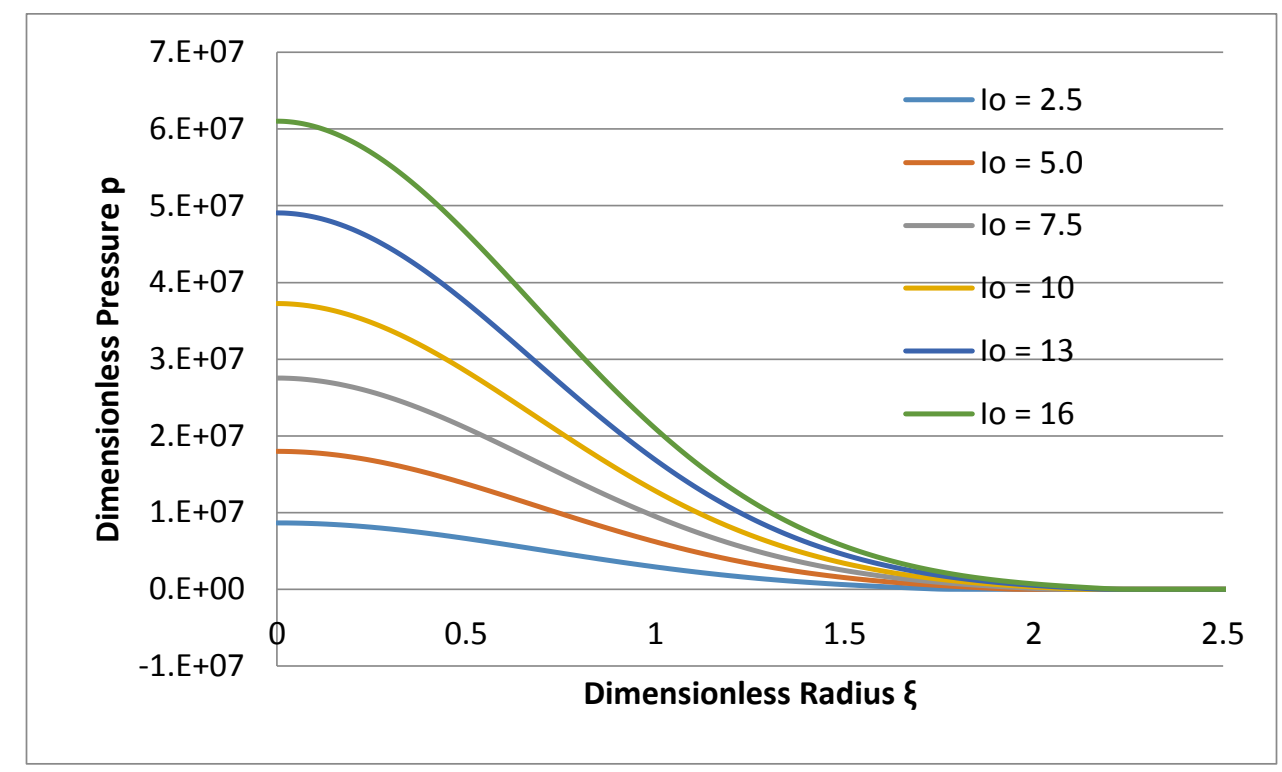

Figure 5.3 Pressure profiles at $\tau=\tau_{p}$ with varying laser beam intensity, predicted from a model assuming rising-fall radial velocity profile with peak velocity at $\xi_{p}=1$. The laser beam intensity is given at the center with unit $10^{10} \mathrm{w} / \mathrm{m}^{2}$.

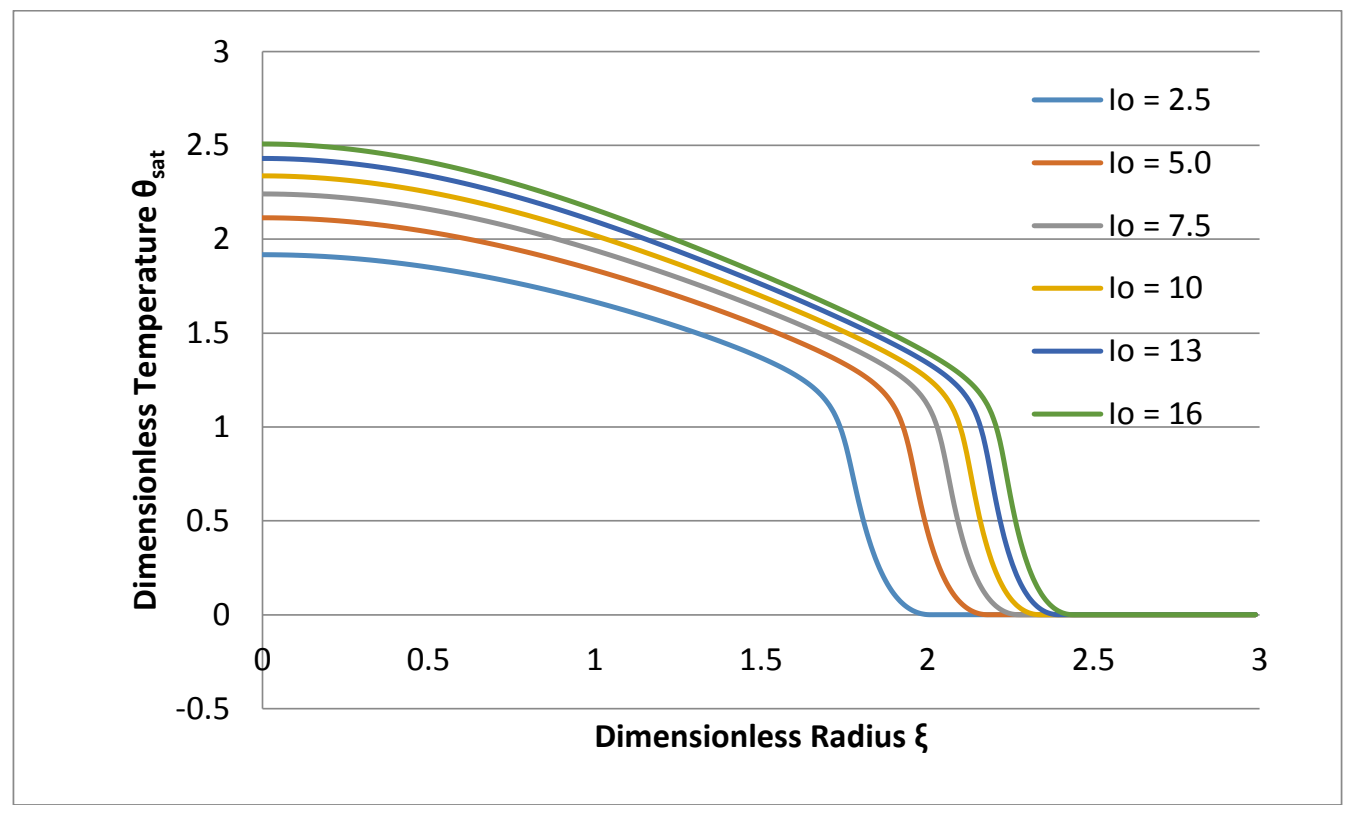

Figure 5.4 Temperature profiles at $\tau=\tau_{p}$ with varying laser beam intensity, predicted from a model assuming a rising-fall radial velocity profile with a peak velocity at $\xi_{p}=1$. The laser beam intensity is given at the center with unit $10^{10} \mathrm{w} / \mathrm{m}^{2}$. 

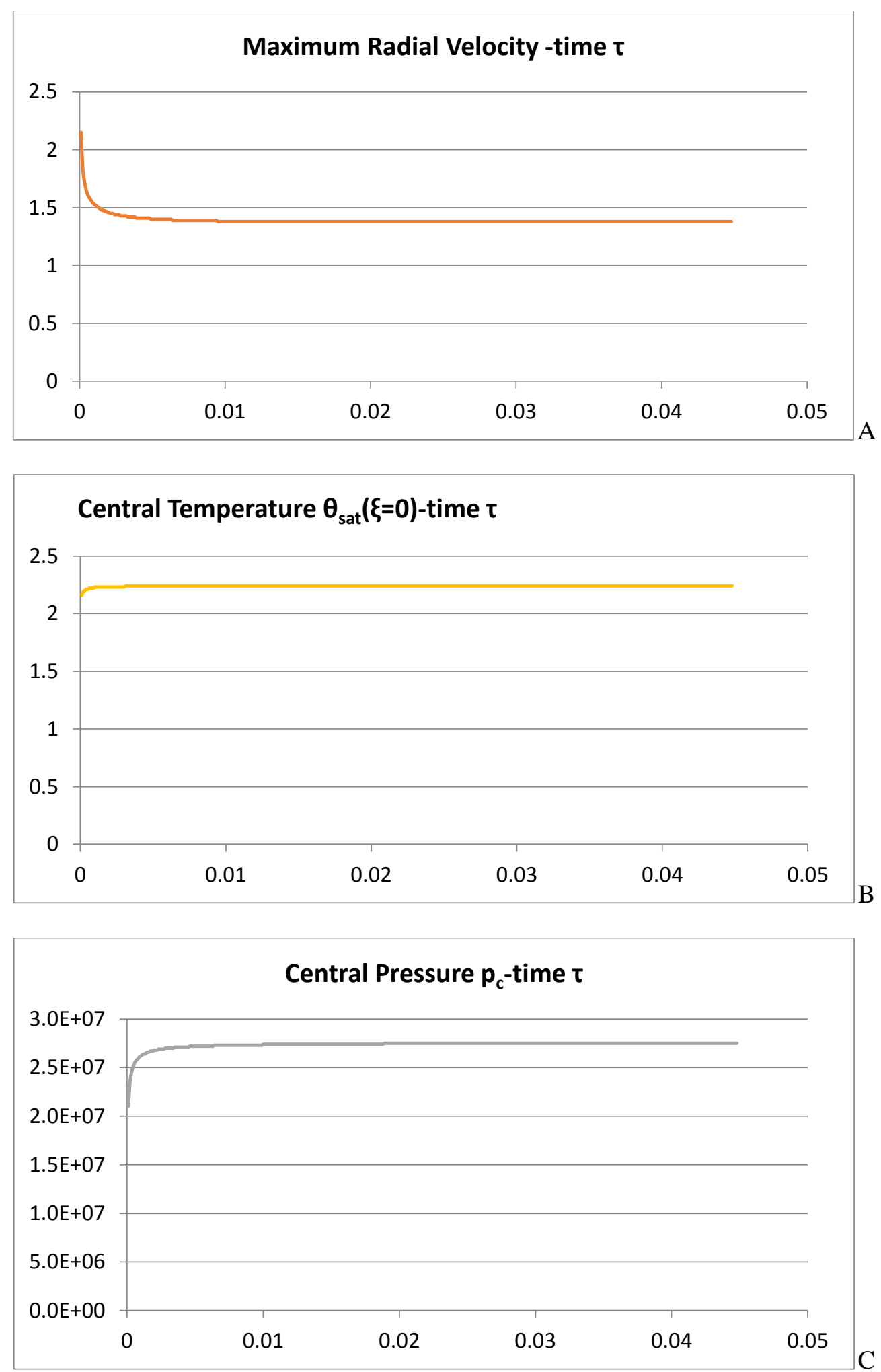

Figure 5.5 Peak radial velocity, temperature and pressure history predicted from a model assuming rising radial velocity profile with index $m=2$. 

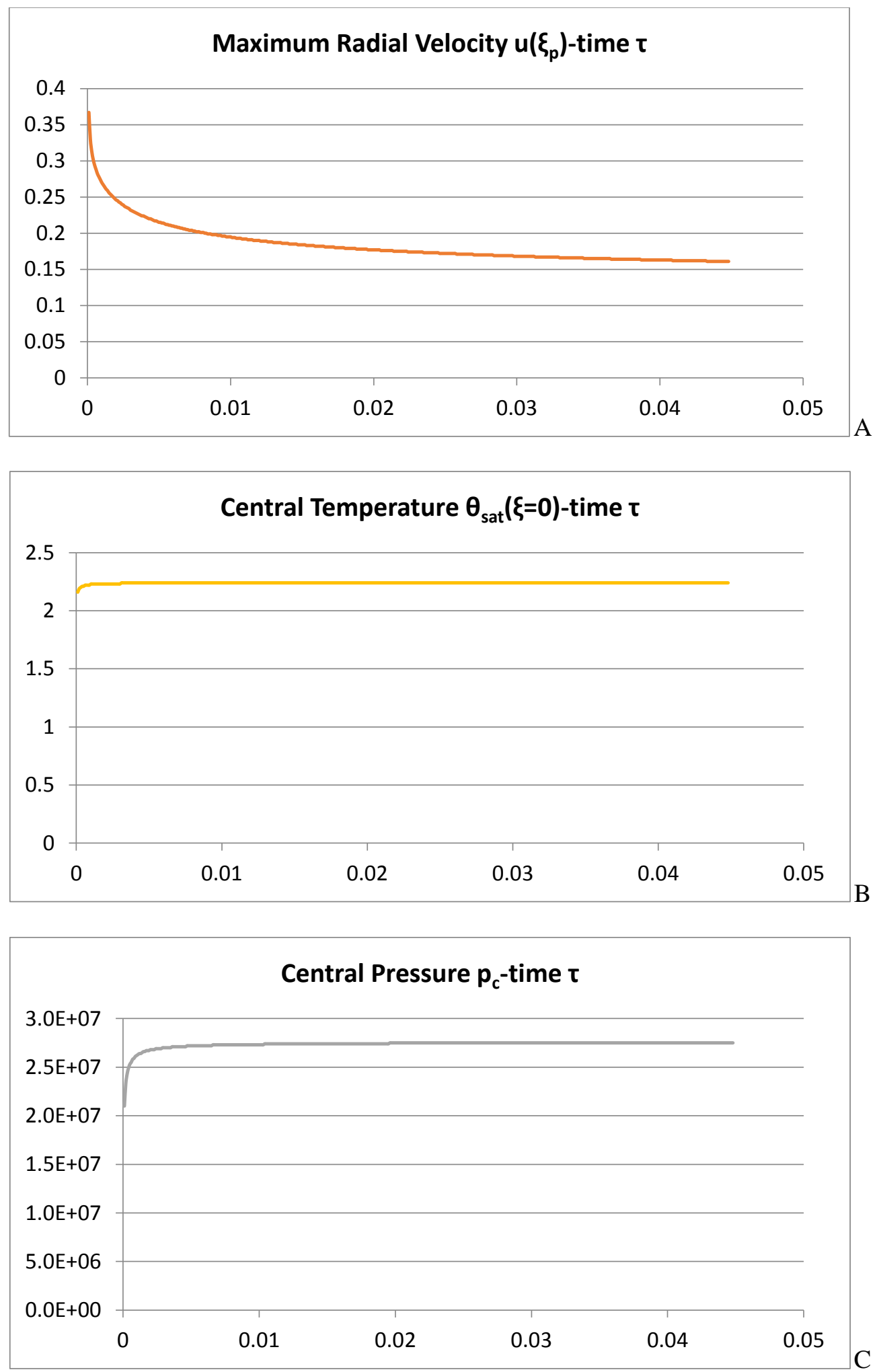

Figure 5.6 Peak radial velocity, temperature and pressure history predicted from a model assuming rising-fall radial velocity profile with peak velocity at $\xi_{p}=0.6$. 

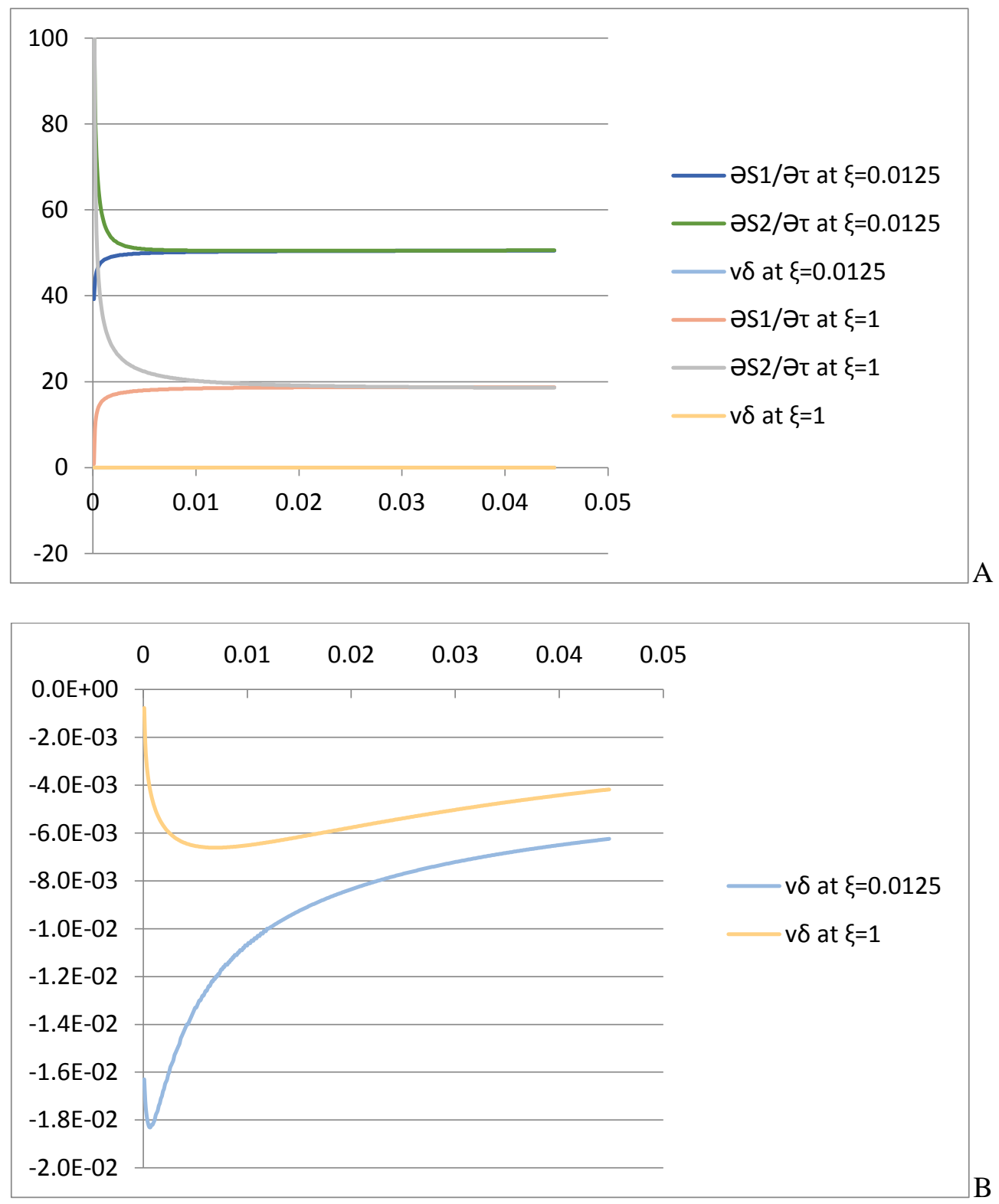

Figure 5.7 (A) Melting rate, vaporization rate and the effect of the melt flow on interfacechange near the laser beam center at $\xi=0.0125$ and at $\xi=1$. (B) The change of flow velocity vertical component at both locations. 

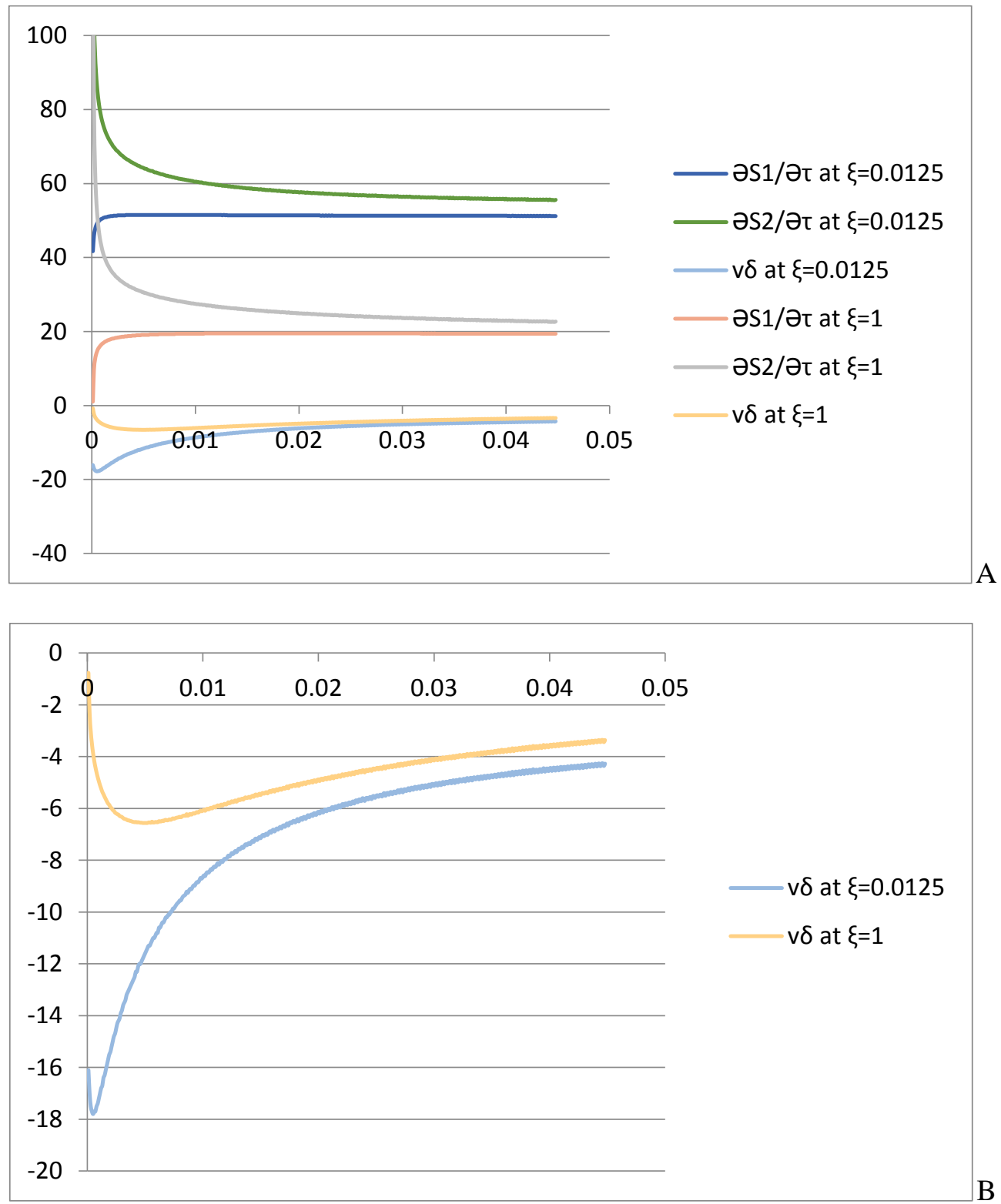

Figure 5.8 (A) Melting rate, vaporization rate and the effect of the melt flow on interface change near the laser beam center $\xi=0.0125$ and at $\xi=1$. (B) The change of flow velocity vertical component at both locations, similar to Figure 5.7 but with a Prattle number 1000 times larger. 


\section{Part II. Combined MPM-DEM for simulating the interaction between solid elements and fluid particles}




\section{Chapter 6. Numerical Simulations on Solid-Fluid Interaction}

Interaction between a large number of solid elements (bodies or particles) and surrounding fluid occurs in many natural and engineering settings such as sediment transport in rivers and coastal oceans, debris flows during flooding, cuttings transport in petroleum-well drilling, mineral particle size reduction in grinding mills, as well as powder handling and pneumatic conveying in pharmaceutical industries (Sun and Xiao 2015). In the category of dense granular flow, the collisions between solid elements and shear drag from surrounding fluid play a dominant role. As for the study of the interaction between solid elements, discrete element method (DEM) is one of the most efficient approaches and has found a firm place in powder mechanics and rock mechanics (Campbell 1986, Zhu, Zhou et al. 2007, Weerasekara, Powell et al. 2013).

The interaction between solid particles and surrounding fluid may be completely ignored or explicitly included in numerical study. On one hand, fluid plays a very important role in fine granular material transfer in some industrial applications. Significant amount of water is added in the grinding chamber to improve the transport of fine material by forming slurry and to suppress dust and to modify the grinding conditions. Such slurry is much more efficient than air for transporting small solid particles out of a mill. Slurry flow, including flow through the charge, through the discharge grates, along the pulp lifters and its discharge from the mill all contribute to the efficiency of the grinding process within a device like a Semi-Autogenous grinding (SAG) mill or an Autogenous grinding mill. Poor transport of finer ground material can adversely affect grinding leading to excess energy consumption and over-grinding of fine material. On the other hand, the size of fine solid particles and the interaction between different phases pose a great challenge to current modeling and simulation (Cleary 2015). 
Because the flow of these materials is difficult to measure and directly observe, computational modelling provides a powerful way of virtually exploring the process, obtaining quantitative process data, and understanding the fundamental physics that will become the source of future design guidance. There are two basic approaches that can be used for simulating slurries. In the first approach, the fine particles are averaged to give a continuum representation with a complex rheological behavior. The governing equations are typically solved using a Finite Volume (FV) or Finite Element Method (FEM). This type of method can be highly effective when the particle sizes are small compared to other length scales in the process, such as a valve caliber or grate size, and when inter-particle contact or collisions are not a dominant manner of momentum transport. Examples of such modelling include erosion by slurry flow in pipes (Brown 2002, Zhang, Reuterfors et al. 2007), pneumatic and slurry flows (Solnordal, Wong et al. 2013), and impinging jet on a plate (Gnanavelu, Kapur et al. 2011).

The alternative approach is to resolve the individual particles in the simulation. The method that enables collision/contact based equations of motion for the particles to be solved is the Discrete Element Method (DEM). In most coarse particle applications, the fine slurry phase is simply omitted by assuming that the fluid coupling forces are small compared to the contact forces and so can be neglected. Careful validation of this assumption is required, but in most current simulations it is missing.

Discrete element method (DEM) is any of a family of numerical methods for computing the motion and effect of a large number of small particles. It is originally proposed by Cundall in 1971 (Cundall and Strack 1979). The earliest use of DEM in comminution was the prediction in two dimensions of charge motion in ball mills by Mishra and Rajamani (Mishra and Rajamani 1992, Mishra and Rajamani 1994, Rajamani and Mishra 1996). DEM simulations take some account of 
multi-particle interactions and can provide useful qualitative information like the trajectory of the solid particles that helps to design a mill of less wasteful collisions like grinding steel ball-liner collision. Three dimensional models of thin slices of the cylinder shell of tumbling mills were used by Herbst and Nordell (Herbst and Nordell 2001) and Cleary (Cleary 2001) to predict the dynamics of charge motion. Such predictions for dry mills were shown to have good accuracy for a range of mill speeds and fill levels (Herbst and Potapov 2004). Full three dimensional modelling of pilot scale SAG mills has also been performed (Cleary, Sinnott et al. 2008, Cleary, Sinnott et al. 2009). It has also been used for modelling of axial flow in ball mills (Cleary, Sinnott et al. 2006) and generally for understanding performance in tumbling mills (Djordjevic 2003, Djordjevic 2005, Kalala, Breetzke et al. 2008, Powell, Weerasekara et al. 2011). It has also been used for modelling of stirred mills (Sinnott, Cleary et al. 2006, Yang, Jayasundara et al. 2006, Jayasundara, Yang et al. 2008).

If the gas or liquid component of a multiphase flow sufficiently influences the coarse particle motion, then the interaction force from the fluid phase has to be explicitly included in the computational model. Tsuji et al. (Tsuji, Kawaguchi et al. 1993) were the first group to propose coupling DEM with an Eulerian fluid model, which was applied to a two-dimensional gas-solid fluidized bed of spherical particles. This type of approach was also used by $\mathrm{Xu}$ and $\mathrm{Yu}$ (Xu and Yu 1997) and Kawaguchi et al. (Kawaguchi, Sakamoto et al. 2000). Kafui et al. (Kafui, Thornton et al. 2002) used a similar model incorporating particle contact mechanics to investigate multiphase interaction. The coupling is achieved by averaging the DEM particle data to give continuum representations of the particle phase and then to use these in continuum multiphase CFD models. Since the particles are averaged and do not explicitly appear in the fluid solution, this type of method is sometimes referred to as unresolved. This type of approach has become very 
popular in modelling gas particle systems such as fluidized beds (Mikami, Kamiya et al. 1998, Hoomans, Kuipers et al. 2000, Yu and Xu 2003, Kafui and Thornton 2008, Tsuji, Yabumoto et al. 2008).

Spherical shape particles are widely used in solid-liquid simulations for its simplicity. Nonround particles are demonstrated in a fluid beds simulation and then in pneumatic conveying simulation (Hilton, Mason et al. 2010, Hilton and Cleary 2011). For the gas coupled flows the Finite Volume (FV) method dominates because of its speed and suitability for solving the multiphase continuum equations.

The prediction of slurry flow in mills and through screens is more computationally challenging because a great number of triangles is needed for describing the motion of complex shaped structure surfaces. In addition, complex free surfaces of the fluid, including splashing and fragmentation, and the resolution of very fine flow features have to be treated properly. Obviously Eulerian grid based flow solvers are not well suited for such requirements. In particular, the fragmenting flow of the many thin streams of fluid passing through the potentially hundreds to thousands of holes in either screens or mill grates can be prohibitively expensive. The fluid method most suited to these applications is Smoothed Particle Hydrodynamics (SPH) since it is a particlebased method; therefore, it naturally allows the prediction of complex splashing and fragmenting free surface flow. This method was originally formulated for free surface fluid dynamics by Monaghan (Potapov, Hunt et al. 2001, Robinson, Ramaioli et al. 2014). The smoothed-particle hydrodynamics (SPH) method has been developed to simulate the motion of fluid (Potapov, Hunt et al. 2001, Robinson, Ramaioli et al. 2014). The SPH method was originally introduced to simulate the problems in astrophysics, which involve fluid particles moving arbitrarily in the absence of boundaries (Monaghan 1988, Monaghan 1992). It has been broadly adopted for 
modelling free surface fluid applications, including die casting (Cleary 2000, Ha and Cleary 2005), flow over a spillway (Ferrari 2010), sloshing (Guzel, Prakash et al. 2005, Shao, Li et al. 2012) and immiscible and miscible fluid mixing (Tartakovsky and Meakin 2006).

In coupling DEM and SPH to simulate slurries, there is a further choice to be made. The fluid method can be used to simulate the detailed flow around each of the particles. This method is sometimes referred to as a resolved method since it resolves the particles and the fluid flow in between. It was first used by Potapov et al. (Potapov, Hunt et al. 2001) for pipe flow. It has also been used by Prakash et al. (Prakash, Cleary et al. 2007) for validating predictions of mixing pellets with water. Although this method produces accurate results and does not rely on any empirical data input, the cost of resolving the fluid flow is very high for a few particulates. It is impossible to extend this method for cases that involve thousands and millions particles. The alternative is to use an unresolved approach, but with the SPH method being used for the fluid flow solution rather than the FV method. This enables large numbers of small solid particles with broad size distributions to be used in the DEM component whilst having a computationally feasible fluid solution that can handle complex moving geometries and fluid free surfaces. Such an approach was proposed using a 1-way coupling between phases Cleary et al. (Cleary 2006). This was subsequently extended to three dimensions and applied to the prediction of slurry flow in a tower mill (Sinnott, Cleary et al. 2011). It has also been used to predict slurry flow on a double deck vibrating banana screen (Fernandez, Cleary et al. 2011). The method was also recently applied to a pilot mill enabling prediction of the free fluid flow within a SAG mill grinding chamber, flow through the grate and discharge flow along the pulp lifters (Cleary and Morrison 2011, Lichter, Suazo et al. 2011, Rajamani, Alkac et al. 2011, Cleary and Morrison 2012). 
In the one-way coupling DEM-SPH simulation, one phase contributing to the motion of others while the reaction from others is ignored (Gao and Herbst 2009, Sinnott, Cleary et al. 2011). Compared with contact force, the interaction from water or air is quite small, but the reaction from solid phase plays a significant role in the motion of the fluid; this is one-way coupling, which is not a full solution, but a simple approach. In many other cases two way coupling is required. Recent research focus has been on the two-way coupling (Chu, Kuang et al. 2012, Cleary 2015). The verification and validation of the combined DEM-SPH has been recently conducted (Sun, Sakai et al. 2013). However, the SPH solution is unstable or not convergent in some cases (Belytschko, Guo et al. 2000, Dehnen and Aly 2012). Hence, further improvement is required on the SPH, or an alternative approach must be found.

It is proposed in this paper that the material point method (MPM) be combined with the DEM to simulate the interaction between solid elements of different sizes and surrounding fluid particles. The MPM is an extension of the particle-in-cell (PIC) method in CFD to the field of computational solid dynamics (CSD), and is a particle method formulated with the same weak formulation as that used for the finite element method (FEM) (Sulsky, Chen et al. 1994). Because the deformation history is recorded with material points for history-dependent constitutive equations, the MPM is able to handle the problems with large deformation, failure evolution, and multi-phase interactions, such as impact, penetration, perforation, and fluid-structure interaction with strong shocks, as demonstrated in previous studies (York Ii, Sulsky et al. 2000, Chen, Shen et al. 2005, Zhang, Zou et al. 2008, Zhang, Wang et al. 2009, Mackenzie-Helnwein, Arduino et al. 2010, Tran, Kim et al. 2010, Gan, Chen et al. 2011, Lian, Zhang et al. 2013, Bandara and Soga 2015, Liu, Liu et al. 2015). In addition, the MPM has been combined with the FEM to improve the computational efficiency (Zhang, Sze et al. 2006). Another approach to improve the computational efficiency is to combine 
the MPM with the adaptive mesh refinement (AMR) techniques. This approach has been adopted to simulate fluid-structure interaction problems (Mao 2013). The MPM has also been employed in multi-scale simulation (Jiang, Chen et al. 2015). A recent work shows that MPM is capable of simulating slosh (Li, Hamamoto et al. 2014). By using the advantages of both the MPM and DEM, an effective spatial discretization procedure might be designed to better simulate the interactions among various kinds of solid elements with surrounding fluid particles.

Although the concept of particles are used in the MPM and the SPH, it is worthy to be aware of their difference. The MPM is developed to seek a numerical solution based on a week form of the differential equations, similar to Finite Element Method. Shape functions are used to complete interpolation and gradient operation. The SPH is a particle based method and kernel functions are utilized to calculate the interpolation and gradient.

In the following chapters, the derivation of the governing equations are presented. The discretization with a corresponding algorithm is then laid out next. The convergence of numerical solutions is demonstrated with typical numerical tests with varying number of particles in a cell and the change of cell resolution. The proposed procedure is verified with three representative examples. The concluding remarks and future work are given at the end. 


\section{Chapter 7. Governing Equations}

Let $\theta$ be the volume fraction of the fluid, $\rho_{f}$ the density of the fluid, and $\boldsymbol{u}_{f}$ the velocity of the fluid. The fluid phase is continuous and the solid phase is discrete. Take a free body diagram shown in Figure 9.1. Let the fluid section of boundary be $A_{f}$, the boundary crossing the solid particles be $A_{p}$, the surface of solid particles inside the control volume $V$ be $\Sigma$, and the surface of solid particles crossing boundary be $\Sigma^{\prime}$.

\subsection{Continuity equations}

Applying mass conservation law for the fluid inside a control volume $V$ with boundary $A$, in the case where no fluid is generated or consumed, one gets

$$
\frac{\partial}{\partial t} \int_{V} \theta \rho_{f} d V+\int_{A}\left(\theta \rho_{f} \boldsymbol{u}_{f}\right) \cdot \boldsymbol{n} d A=0
$$

where $\boldsymbol{n}$ denotes the outward normal vector on the boundary of control volume $V, A=A_{p}+A_{f}$.

According to the divergence theorem, the above equation can be rewritten as

$$
\int_{V} \frac{\partial\left(\theta \rho_{f}\right)}{\partial t} d V+\int_{V} \nabla \cdot\left(\theta \rho_{f} \boldsymbol{u}_{f}\right) d V=0
$$

Since the control volume $V$ is arbitrary, we have

$$
\frac{\partial\left(\theta \rho_{f}\right)}{\partial t}+\nabla \cdot\left(\theta \rho_{f} \boldsymbol{u}_{f}\right)=0
$$

which is similar with the formula of the continuous single phase in (Brennen 2005).

\subsection{Momentum equations}

Applying Newton's second law of motion to the fluid part within the control volume $V$, we get

$$
\int_{V}\left(\theta \rho_{f} \frac{D \boldsymbol{u}_{f}}{D t}\right) d V=\int_{V} \theta \rho_{f} \boldsymbol{g} d V+\int_{A_{f} \cup \Sigma^{\prime} \cup \Sigma} \boldsymbol{\sigma n} d A
$$


where $\boldsymbol{\sigma}$ is the stress tensor, $\boldsymbol{n}$ is the outward normal vector at $A_{f}$, or pointing to the solid particles at $\Sigma^{\prime} \cup \Sigma$. The fluid pressure $p$, stress $\boldsymbol{\sigma}$ and deviatoric stress $\boldsymbol{\tau}$ have the following relationship.

$$
\boldsymbol{\sigma}=-p \boldsymbol{I}+\boldsymbol{\tau}
$$

We break the second term on the right hand side of eq. (9-4) by applying (9-5),

$$
\int_{A_{f} \cup \Sigma^{\prime} \cup \Sigma} \boldsymbol{\sigma n} d A=\int_{A_{f} \cup A_{p}} \boldsymbol{\sigma} \boldsymbol{n} d A-\int_{\Sigma^{\prime} \cup \Sigma} \boldsymbol{\eta n} d A+\int_{A_{p}} \boldsymbol{p} \boldsymbol{n} d A+\int_{\Sigma^{\prime} \cup \Sigma} \boldsymbol{\tau n} d A-\int_{A_{p}} \boldsymbol{\tau n} d A
$$

An empirical formula (Ergun 1952, Kawaguchi, Tanaka et al. 1995, Kawaguchi, Tanaka et al. 1998, Sun, Sakai et al. 2013) is used to calculate the contribution of the deviatoric stress at the solid-fluid interface:

$$
\int_{\Sigma^{\prime} \cup \Sigma} \boldsymbol{\tau} \boldsymbol{n} d A-\int_{A_{p}} \boldsymbol{\tau n} d A=\sum_{j=1}^{m} \beta_{j}\left(\boldsymbol{v}_{s}\left(\boldsymbol{x}_{j}\right)-\boldsymbol{u}_{f}\left(\boldsymbol{x}_{j}\right)\right) V_{j},
$$

where $m$ is the number of solid particles in the control volume, $\boldsymbol{x}_{j}$ is the coordinate of the mass center of the j-th solid particle, $\boldsymbol{v}_{s}$ is the velocity of solid particle, and $\boldsymbol{u}_{f}$ is the fluid velocity, which can be regarded as an averaged value of fluid velocity around the solid particle, equivalent to the velocity at the mass center as if the space occupied by the solid particle were filled with the fluid. $\beta_{j}$ is the drag coefficient and $V_{j}$ the volume of the $\mathrm{j}$-th solid particle.

Similar to Eq. (7-7), the concentrated force is to represent the resultant force due to the pressure on the solid particle surface, that is, a solid particle is processed either in one cell or another, but not in between. The pressure terms on the right hand side of eq. (7-6) can be rewritten by using the divergence theorem as: 


$$
\begin{aligned}
& -\int_{\Sigma^{\prime} \cup \Sigma} p \boldsymbol{n} d A+\int_{A_{p}} p \boldsymbol{n} d A=-\int_{\Sigma_{1}} p \boldsymbol{n} d A-\cdots-\int_{\Sigma_{m}} p \boldsymbol{n} d A=\int_{V_{1}} \nabla p d V+\cdots+\int_{V_{m}} \nabla p d V \approx \\
& \nabla p\left(\boldsymbol{x}_{1}\right) V_{1}+\cdots+\nabla p\left(\boldsymbol{x}_{m}\right) V_{m}=\sum_{j=1}^{m} \nabla p\left(\boldsymbol{x}_{j}\right) V_{j},
\end{aligned}
$$

where $m$ is the number of solid particles in a control volume $V, \Sigma_{1}, \ldots, \Sigma_{m}$ are the surfaces of the solid particles.

Although the left side of Eq. (7-6) takes into account the solid particles crossing the border of the control volume, the right side contains only the whole particles, for all solid particles are treated either in one control volume or another, but not in between. This approach is only for the purpose of calculating the resultant force. The effect of the position of the solid particle mass center will be counted in the MPM. In this way, the pressure term and the deviatoric stress term are treated consistently. The first term on the right hand side of Eq. (7-6) can be rewritten by applying the divergence theorem as

$$
\int_{A_{f} \cup A_{p}} \boldsymbol{\sigma n} d A=\int_{V} \nabla \cdot \boldsymbol{\sigma} d V
$$

Combining (7-4), (7-6) and (7-9), one gets

$$
\int_{V} \theta \rho_{f} \frac{D \boldsymbol{u}_{f}}{D t} d V=\int_{V} \theta \rho_{f} \boldsymbol{g} d V+\int_{V} \nabla \cdot \boldsymbol{\sigma} d V+\sum_{j=1}^{m} \nabla p\left(\boldsymbol{x}_{j}\right) V_{j}+\sum_{j=1}^{m} \beta_{j}\left(\boldsymbol{v}_{s}\left(\boldsymbol{x}_{j}\right)-\boldsymbol{u}_{f}\left(\boldsymbol{x}_{j}\right)\right) V_{j}
$$

To get the differential form of the governing equation, we use Dirac delta function to represent the distribution of the concentrated forces.

$$
\sum_{j=1}^{m} \nabla p\left(\boldsymbol{x}_{j}\right) V_{j}=\int_{V} \sum_{j=1}^{m} \nabla p(\boldsymbol{x}) V_{j} \delta\left(\boldsymbol{x}-\boldsymbol{x}_{j}\right) d V
$$




$$
\sum_{j=1}^{m} \beta_{j}\left(\boldsymbol{v}_{s}\left(\boldsymbol{x}_{j}\right)-\boldsymbol{u}_{f}\left(\boldsymbol{x}_{j}\right)\right) V_{j}=\sum_{j=1}^{m} \int_{V} \beta_{j}\left(\boldsymbol{v}_{s}(\boldsymbol{x})-\boldsymbol{u}_{f}(\boldsymbol{x})\right) V_{j} \delta\left(\boldsymbol{x}-\boldsymbol{x}_{j}\right) d V
$$

Substituting (7-11) and (7-12) into (7-10), we have

$$
\begin{aligned}
\int_{V} \theta \rho_{f} \frac{D \boldsymbol{u}_{f}}{D t} d V & \\
& =\int_{V}\left\{\theta \rho_{f} \boldsymbol{g}+\nabla \cdot \boldsymbol{\sigma}\right. \\
& \left.+\sum_{j=1}^{m}\left[\nabla p(\boldsymbol{x}) V_{j} \delta\left(\boldsymbol{x}-\boldsymbol{x}_{j}\right)+\beta_{j}\left(\boldsymbol{v}_{s}(\boldsymbol{x})-\boldsymbol{u}_{f}(\boldsymbol{x})\right) V_{j} \delta\left(\boldsymbol{x}-\boldsymbol{x}_{j}\right)\right]\right\} d V
\end{aligned}
$$

Since the domain $V$ in the above equation is arbitrary, to make two integrals equal we shall require the integrands on both side equal. Thus, we get the general form of governing equation in the differential form as follows

$$
\theta \rho_{f} \frac{D \boldsymbol{u}_{f}}{D t}=\theta \rho_{f} \boldsymbol{g}+\nabla \cdot \boldsymbol{\sigma}+\sum_{j=1}^{M}\left[\nabla p(\boldsymbol{x})+\beta_{j}\left(\boldsymbol{v}_{s}-\boldsymbol{u}_{f}\right)\right] V_{j} \delta\left(\boldsymbol{x}-\boldsymbol{x}_{j}\right)
$$

where $M$ is the number of solid particles in the whole computational domain. If the interaction force from the solid phase is represented by $\boldsymbol{R}$, i.e. $\boldsymbol{R}=\sum_{j=1}^{M}\left[\nabla p(\boldsymbol{x})+\beta_{j}\left(\boldsymbol{v}_{s}-\boldsymbol{u}_{f}\right)\right] V_{j} \delta\left(\boldsymbol{x}-\boldsymbol{x}_{j}\right)$, Eq. (7-14) becomes the same form of momentum conservation equation of a continuous single phase derived in (Brennen 2005).

In the combined DEM-MPM, the motion of a solid particle is based on the resultant force the particle receives, including the contribution from the fluid field. The shear from the fluid is the 
reaction force of $\beta_{j}\left(\boldsymbol{v}_{s}\left(\boldsymbol{x}_{j}\right)-\boldsymbol{u}_{f}\left(\boldsymbol{x}_{j}\right)\right) V_{j}$, and the pressure on a solid particle is the buoyance force, which can be calculated as

$$
\int_{\Sigma_{j}} p \boldsymbol{n} d A=-\int_{V_{j}} \nabla p d V \approx-V_{j} \nabla p\left(\boldsymbol{x}_{j}\right)
$$

where $\Sigma_{j}$ and $V_{j}$ are the surface and volume of the j-th solid particle, respectively.

The resultant force on fluid phase from the solid particle $j$ is marked as $\boldsymbol{f}_{f}$, defined as

$$
\boldsymbol{f}_{f}=V_{j}\left\{\nabla p\left(\boldsymbol{x}_{j}\right)+\beta_{j}\left[\boldsymbol{v}_{s}\left(\boldsymbol{x}_{j}\right)-\boldsymbol{u}_{f}\left(\boldsymbol{x}_{j}\right)\right]\right\}
$$

The average reaction force of the buoyance force (exerted on the fluid phase) per unit volume in the control volume can be estimated as follows, for the case in which the gradient of pressure is constant over each solid particle.

$$
\frac{1}{V} \int_{V} \sum_{j=1}^{m} \nabla p(\boldsymbol{x}) V_{j} \delta\left(\boldsymbol{x}-\boldsymbol{x}_{j}\right) d V=\frac{1}{V} \sum_{j=1}^{m} \nabla p\left(\boldsymbol{x}_{j}\right) V_{j}=\frac{\nabla p}{V} \sum_{j=1}^{m} V_{j}=\nabla p(1-\theta)
$$

Here the locally averaged solid volume fraction over the control volume, $\frac{1}{V} \sum_{j=1}^{m} V_{j}=1-\theta$, is applied. Since $\sum_{j=1}^{m} \nabla p(\boldsymbol{x}) V_{j} \delta\left(\boldsymbol{x}-\boldsymbol{x}_{j}\right)$ represents the force per unit volume, and has the same resultant force over the fluid inside the control volume, (i.e. $\nabla p(1-\theta) V$ ), in the case where the solid particles are very tiny and control volume is very small, so that the constant gradient of pressure would approximately hold. Mathematically, the gradient of pressure is independent of the location in the control volume as the control volume approaches infinitesimal. The Eq. (7-14) is thus rewritten as

$$
\begin{aligned}
& \theta \rho_{f} \frac{D \boldsymbol{u}_{f}}{D t}=\theta \rho_{f} \boldsymbol{g}+\nabla \cdot \boldsymbol{\sigma}+\nabla p(1-\theta)+\sum_{j=1}^{M} \beta_{j}\left(\boldsymbol{v}_{s}-\boldsymbol{u}_{f}\right) V_{j} \delta\left(\boldsymbol{x}-\boldsymbol{x}_{j}\right)=\theta \rho_{f} \boldsymbol{g}-\theta \nabla p+ \\
& \nabla \cdot \boldsymbol{\tau}+\sum_{j=1}^{M} \beta_{j}\left(\boldsymbol{v}_{s}-\boldsymbol{u}_{f}\right) V_{j} \delta\left(\boldsymbol{x}-\boldsymbol{x}_{j}\right)
\end{aligned}
$$


If $\boldsymbol{F}_{f}$ is used to represent the shear drag force per unit volume on fluid phase, which is the last term in the above equation, we get the exact same form of momentum equation as derived by Anderson and Jackson (Anderson and Jackson 1967), though our derivation is much shorter and with fewer assumptions. In many applications, the shear drag force is actually calculated using formula (5), but an abstract symbol $\boldsymbol{F}_{f}$ or a term like $\beta\left(\boldsymbol{v}_{s}-\boldsymbol{u}_{f}\right)$ appears in the momentum conservation equation. The connection between $\boldsymbol{F}_{f}$ and the shear drag is missing. And, $\beta\left(\boldsymbol{v}_{s}-\boldsymbol{u}_{f}\right)$ does not reflect the essence of the empirical formula, which basically is an estimate of a resultant force concentrated at the mass center. By using the Dirac delta function here, the nature of shear drag as an empirically determined resultant force concentrated at the mass point of dispersed solid phase is thus correctly represented in the momentum conservation equation.

\subsection{Constitutive equations}

The continuity equation (7-3) can be rewritten as

$$
\frac{D\left(\theta \rho_{f}\right)}{D t}=-\theta \rho_{f} \nabla \cdot \boldsymbol{u}_{f}
$$

where $\frac{D}{D t}$ denotes the substantial (material) derivative. Eq. (7-19) can be rewritten as

$$
\frac{D \rho_{f}}{\rho_{f} D t}=-\nabla \cdot \boldsymbol{u}_{f}-\frac{D \theta}{\theta D t}
$$

In the Lagrangian description, $\frac{D \theta}{D t}$ turns out to be $\frac{d \theta}{d t}$ which is the rate of change of fluid volume fraction of a given fluid particle, and $\frac{D \rho_{f}}{D t}$ becomes $\frac{d \rho_{f}}{d t}$ which is the rate of change of fluid density. The fluid particle will be simulated using the MPM described in next section. The density $\rho_{f}$ is 
the fluid particle density, not the density described at a fixed spatial point in the flow field in Eulerian description. In another words, we will use Lagrangian description through this study.

The equation (7-20) can be approximated by a difference form as

$$
\frac{\Delta \rho_{f}}{\rho_{f}}=-\left(\nabla \cdot \boldsymbol{u}_{f}+\frac{1}{\theta} \frac{\Delta \theta}{\Delta t}\right) \Delta t=-\left(\Delta t \nabla \cdot \boldsymbol{u}_{f}+\frac{\theta-\theta_{0}}{\theta}\right)
$$

where $\Delta t$ is the time increment, and $\theta_{0}$ is the fluid volume fraction at the previous time step.

The pressure change is related to the density change through bulk modulus $K$ in a linear relationship, i.e., $\Delta p=K \frac{\Delta \rho_{f}}{\rho_{f}}$, thus we have

$$
\Delta p=-K\left(\Delta t \nabla \cdot \boldsymbol{u}_{f}+\frac{\theta-\theta_{0}}{\theta}\right)
$$

The density of the fluid evolves from $\rho_{f}^{n}$ at the previous time step to $\rho_{f}^{n+1}$ at the current time step as

$$
\rho_{f}^{n+1}=\frac{\rho_{f}^{n}}{1-\frac{\Delta p}{K}}
$$

Hence, the current fluid density may be roughly calculated from the current pressure $p^{k+1}$ through $\rho_{f}^{n+1}=\frac{\rho_{f}^{0}}{1-\frac{p^{n+1}}{K}}$, where $\rho_{f}^{0}$ is the density in the initial condition and the initial pressure is assumed to be zero.

The deviatoric stress $\boldsymbol{\tau}$ is assumed to follow Navier-Poisson Law of a Newtonian fluid:

$$
\boldsymbol{\tau}=2 \eta \dot{\boldsymbol{\varepsilon}}_{f}^{d}
$$

where $\dot{\varepsilon}_{f}^{d}$ is the deviatoric strain rate and $\eta$ is the dynamic viscosity of the fluid. 


\subsection{The equation of solid particle motion}

The motion of solid particles is considered at the level of each individual particle. The motion of mass center of each individual particle follows Newton's second law of motion for a single mass point, and the rotation of the particle follows Euler's Theorem, which is an extension of Newton's law to the motion of a rigid body.

$$
\begin{aligned}
& m_{s} \frac{d v_{s}}{d t}=\boldsymbol{f}_{c}-\boldsymbol{f}_{f}+m_{s} \boldsymbol{g} \\
& \boldsymbol{I}_{c} \frac{d \boldsymbol{\omega}_{s}}{d t}+\boldsymbol{\omega}_{s} \times\left(\boldsymbol{I}_{c} \boldsymbol{\omega}_{s}\right)=\boldsymbol{T}_{c}+\boldsymbol{T}_{f},
\end{aligned}
$$

where, $m_{s}, \boldsymbol{g}, \boldsymbol{f}_{c}$ are the mass of the solid particle, gravitational acceleration, and the reaction force due to contact (collision) with other solid particles or solid structures, respectively. The method for calculating $\boldsymbol{f}_{c}$ is the so called linear spring-dashpot model proposed by Cundall and Strack (Cundall and Strack 1979), where the spring is used for the elastic deformation while the dashpot accounts for the viscous dissipation and the damping factor is determined from a restitution coefficient provided. $\boldsymbol{I}_{c}$ is momentum of inertia relative to the particle mass center, and $\boldsymbol{\omega}_{s}$ is the angular velocity of the solid particle. $\boldsymbol{T}_{c}$ is the torque due to the contact force from other solid particles or solid structures. $\boldsymbol{T}_{f}$ is the torque due to the interaction with fluid phase. Because only force at the mass center of each solid particle is calculated, the torque relative to the mass center of the solid particle due to the interaction with fluid is neglected at the current model.

The motion of the solid particles is solved using the Discrete Element Method (DEM). The solid particle velocity and fluid velocity at the previous time step are used for calculating the shear drag at the current time step. The solid particle velocity is updated by solving the above equation. The angular velocity is used for calculating the shear force at the contact. The shear force is limited 
by applying Coulomb's friction law. The contact force is calculated based on the overlap between solid particles or solid structures using a viscous damped free vibration model. Further details can be found in the literature (Cundall and Strack 1979, Tsuji, Kawaguchi et al. 1993). 


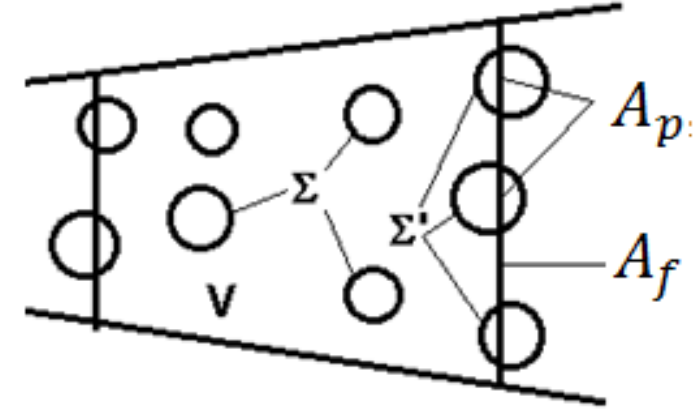

(A)

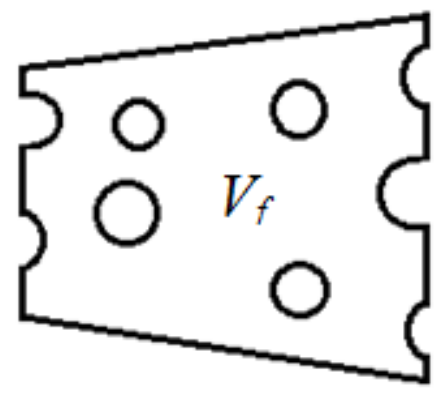

(B)

Figure 7.1. (A) Control volume $V$ with fluid and particles, (B) Fluid volume $V_{f}$, excluding solid volume; modified after (Jin, Yuan et al. 1992) 


\section{Chapter 8. Discretization of Governing Equations}

The material point method (MPM) is exploited to solve the above governing equations, in particular, the momentum balance equation (7-14).

Now we assume a trial function $h$ in the form

$$
h(\boldsymbol{x}, t)=\sum_{j=1}^{N} h_{j}(t) S_{j}(\boldsymbol{x}),
$$

where, $h_{j}(t)$ is a function of time and $S_{j}(\boldsymbol{x})$ a function of spatial coordinate. The trial function $h(\boldsymbol{x}, t)$ consists of $N$ products of these two basic functions. When this trial function is applied to

an element of $N$ nodes, $h_{j}(t)$ is the solution at $\mathrm{j}$-th node varying with time, and $S_{j}(\boldsymbol{x})$ is the spatial interpolation function.

Multiplying (8-1) to (7-14), and then integrating the resulting equation over a computational domain $\Omega$, one gets

$$
\begin{gathered}
\sum_{j=1}^{N} h_{j}(t) \int_{\Omega} \theta \rho_{f} \frac{D \boldsymbol{u}_{f}}{D t} S_{j}(\boldsymbol{x}) d v=\sum_{j=1}^{N} h_{j}(t) \int_{\Omega} S_{j}(\boldsymbol{x}) \theta \rho_{f} \boldsymbol{g} d v+\sum_{j=1}^{N} h_{j}(t) \int_{\Omega} S_{j}(\boldsymbol{x}) \nabla \cdot \boldsymbol{\sigma} d v+ \\
\sum_{j=1}^{N} h_{j}(t) \int_{\Omega} S_{j}(\boldsymbol{x})\left[\sum_{k=1}^{N_{S p}}\left[\nabla p(\boldsymbol{x})+\beta_{k}\left(\boldsymbol{v}_{s}-\boldsymbol{u}_{f}\right)\right] V_{k} \delta\left(\boldsymbol{x}-\boldsymbol{x}_{k}\right)\right] d v
\end{gathered}
$$

where $N_{s p}$ is the number of solid particles in domain $\Omega$.

\subsection{Material Point Method}

The material point method (MPM) is applied to represent the mass of a fluid particle in a space $\omega$ by a concentrated mass at one point-the mass center as follows.

$$
M_{p}=\int_{\omega} \theta \rho_{f} d \omega
$$


The particle mass $M_{p}$ never changes with time. It can also be regarded as $\theta V_{\omega} \rho_{f}$. The macro density is defined as the mass divided by the total volume, i.e. $\frac{M_{p}}{V_{\omega}}$, which is equivalent to the product of micro density (material density) and volume fraction, shown as follows

$$
\theta \rho_{f}=\frac{M_{p}}{V_{\omega}}
$$

Eq. (8-4) holds only if $\theta \rho_{f}$ is constant in space $\omega$. This requirement can be avoided in the MPM, where, the mass of a material particle is treated as being concentrated at a point (that is why it is termed as the material point method). On the other hand, clearly,

$$
\int_{\omega} M_{p} \delta\left(\boldsymbol{x}-\boldsymbol{x}_{p}^{t}\right) d \omega=M_{p}\left(\boldsymbol{x}_{p}^{t}\right)
$$

where $\boldsymbol{x}_{p}^{t}$ is the coordinate of the material point at time $t$. Comparing (8-3) and (8-5), and considering that $\omega$ is arbitrary, we have

$$
\theta \rho_{f}=M_{p} \delta\left(\boldsymbol{x}-\boldsymbol{x}_{p}^{t}\right)
$$

In the case where there are $N_{p}$ material particles inside the space $\Omega$, by a similar argument, we have

$$
\theta \rho_{f}=\sum_{p=1}^{N_{p}} M_{p} \delta\left(\boldsymbol{x}-\boldsymbol{x}_{p}^{t}\right)
$$

The solution process of the momentum conservation equations will be significantly simplified by using the concept of the material point, as shown below.

Similar to the finite element method, the solution $\boldsymbol{u}_{f}(\boldsymbol{x}, t)$ can be obtained through the interpolation of values at the nodes of a regular grid using shape functions:

$$
\boldsymbol{u}_{f}(\boldsymbol{x}, t)=\sum_{j=1}^{N_{n}} \boldsymbol{u}_{f j}(t) S_{j}(\boldsymbol{x})
$$


where $N_{n}$ is the number of nodes in a grid cell (MPM mesh).

Substituting (8-7) into (8-2), one gets the left side

$$
\begin{gathered}
\sum_{j=1}^{N} h_{j}(t) \int_{\Omega} \theta \rho_{f} \frac{D \boldsymbol{u}_{f}}{D t} S_{j}(\boldsymbol{x}) d \Omega=\sum_{j=1}^{N} h_{j}(t) \int_{\Omega} \sum_{p=1}^{N_{p}} M_{p} \delta\left(\boldsymbol{x}-\boldsymbol{x}_{p}^{t}\right) \frac{D \boldsymbol{u}_{f}}{D t} S_{j}(\boldsymbol{x}) d \Omega= \\
\sum_{j=1}^{N} h_{j}(t) \frac{d}{d t}\left[\sum_{p=1}^{N_{p}} M_{p} \boldsymbol{u}_{f}\left(\boldsymbol{x}_{p}^{t}, t\right) S_{j}\left(\boldsymbol{x}_{p}^{t}\right)\right]=\sum_{j=1}^{N} h_{j}(t) \frac{d \overline{m u}_{f j}}{d t}
\end{gathered}
$$

where,

$$
\overline{m \boldsymbol{u}}_{f j}=\sum_{p=1}^{N_{p}} M_{p} \boldsymbol{u}_{f}\left(\boldsymbol{x}_{p}^{t}, t\right) S_{j}\left(\boldsymbol{x}_{p}^{t}\right)
$$

is the momentum of the fluid particle at grid node, obtained by mapping momentum from material particles. The conversion from $\frac{D \boldsymbol{u}_{f}}{D t}$ to $\frac{d \boldsymbol{u}_{f}}{d t}$ is because the fluid velocity is calculated at the point occupied by the moving fluid particle in Lagrangian description, not a fixed spatial point in Eulerian description.

Equation (10-10) defines a mapping from particles in a cell to the grid nodes of the cell. This technique is also applied to locally calculate the averaged volume fraction of each phase at the grid nodes. The solid phase volume fraction and fluid phase volume fraction are calculated as follows.

$$
\begin{aligned}
& \theta_{s j}=\frac{1}{V_{j}} \sum_{s p=1}^{N_{s p}} V_{s p} S_{j}\left(\boldsymbol{x}_{s p}^{t}\right) \\
& \theta_{j}=\frac{1}{V_{j}} \sum_{p=1}^{N_{p}} \frac{M_{p}}{\rho_{f}} S_{j}\left(\boldsymbol{x}_{p}^{t}\right)
\end{aligned}
$$

where the subscript $s p$ represents the solid particle, $V_{s p}$ is the volume of solid particle $s p$ and $V_{j}$ is the volume of all cells associated with the node $\mathrm{j}$, given as

$$
V_{j}=\int_{\Omega} S_{j}(\boldsymbol{x}) d \Omega
$$


Because $\theta_{s j}+\theta_{j}=1$, the fluid volume fraction can be calculated from the solid particle volume fraction as follows,

$$
\theta_{j}=1-\theta_{s j}
$$

As an inverse operation, the following mapping converts nodal variables to variables at the position of particles.

$$
\begin{aligned}
& p(\boldsymbol{x}, t)=\sum_{j=1}^{N_{n}} p_{j}(t) S_{j}(\boldsymbol{x}), \\
& \theta(\boldsymbol{x}, t)=\sum_{j=1}^{N_{n}} \theta_{j}(t) S_{j}(\boldsymbol{x}), \\
& \boldsymbol{\sigma}(\boldsymbol{x}, t)=\sum_{j=1}^{N_{n}} \boldsymbol{\sigma}_{j}(t) S_{j}(\boldsymbol{x}) .
\end{aligned}
$$

The first item on the right side of Eq. (8-2) is the gravitational force.

$$
\int_{\Omega} \theta \rho_{f} \boldsymbol{g} S_{j}(\boldsymbol{x}) d \Omega=\boldsymbol{g} \int_{\Omega} \sum_{p=1}^{N_{p}} M_{p} \delta\left(\boldsymbol{x}-\boldsymbol{x}_{p}^{t}\right) S_{j}(\boldsymbol{x}) d \Omega=M_{p j} \boldsymbol{g}
$$

where

$$
M_{p j}=\sum_{p=1}^{N_{p}} M_{p}\left(\boldsymbol{x}_{p}^{t}, t\right) S_{j}\left(\boldsymbol{x}_{p}^{t}\right)
$$

is a mapping of mass from particles in a cell to the grid node of the cell.

The second item on the right side of Eq. (10-2) can be rewritten as

$$
\int_{\Omega} S_{j}(\boldsymbol{x}) \nabla \cdot \boldsymbol{\sigma} d \Omega=\int_{S} S_{j}(\boldsymbol{x}) \boldsymbol{\sigma} n d s-\int_{\Omega} \boldsymbol{\sigma} \nabla S_{j}(\boldsymbol{x}) d \Omega
$$

The first term on the right side in the above is related to the boundary condition and is marked as

$$
f_{j}^{b c}=\int_{S} S_{j}(\boldsymbol{x}) \boldsymbol{\sigma} n d s
$$

and the second term is the internal force 


$$
\begin{aligned}
f_{j}^{i n t}=-\int_{\Omega} \boldsymbol{\sigma} \nabla S_{j}(\boldsymbol{x}) d \Omega & =-\int_{\Omega} \rho_{f} \boldsymbol{\sigma}^{\boldsymbol{s}} \nabla S_{j}(\boldsymbol{x}) d \Omega=-\frac{1}{\theta} \int_{\Omega} \sum_{p=1}^{N_{p}} M_{p} \delta\left(\boldsymbol{x}-\boldsymbol{x}_{p}^{t}\right) \boldsymbol{\sigma}^{\boldsymbol{s}} \nabla S_{j}(\boldsymbol{x}) d \Omega= \\
-\frac{1}{\theta} \sum_{p=1}^{N_{p}} M_{p} \boldsymbol{\sigma}^{\boldsymbol{s}}\left(\boldsymbol{x}_{p}^{t}\right) \nabla S_{j}\left(\boldsymbol{x}_{p}^{t}\right) & =-\frac{1}{\theta} \sum_{p=1}^{N_{p}} \frac{M_{p}}{\rho_{f}} \boldsymbol{\sigma}_{p} \nabla S_{j}\left(\boldsymbol{x}_{p}^{t}\right)
\end{aligned}
$$

where, the specific stress $\boldsymbol{\sigma}^{s}=\frac{\sigma}{\rho_{f}}$ is used in the derivation of Eq. (8-22). Eq. (8-7) is also applied to derive Eq. (8-22). Note that the fluid volume fraction is obtained by local averaging at the space $\Omega$

The last term in (10-22) is rewritten as:

$$
\begin{gathered}
\sum_{j=1}^{N} h_{j}(t) \int_{\Omega} S_{j}(\boldsymbol{x})\left[\sum_{k=1}^{N_{s p}}\left[\nabla p(\boldsymbol{x})+\beta_{k}\left(\boldsymbol{v}_{s}-\boldsymbol{u}_{f}\right)\right] V_{k} \delta\left(\boldsymbol{x}-\boldsymbol{x}_{k}\right)\right] d \Omega= \\
\sum_{j=1}^{N} h_{j}(t) \sum_{k=1}^{N_{s p}}\left[\nabla p\left(\boldsymbol{x}_{k}\right)+\beta_{j}\left(\boldsymbol{v}_{s}\left(\boldsymbol{x}_{k}\right)-\boldsymbol{u}_{f}\left(\boldsymbol{x}_{k}\right)\right)\right] S_{j}\left(\boldsymbol{x}_{k}\right) V_{k},
\end{gathered}
$$

where, $V_{k}$ is the volume of solid particle $k$. Since the solid particles are regarded as rigid, the volume can be calculated either as $V_{k}=\frac{m_{s k}}{\rho_{s k}}$ or $V_{k}=\frac{4}{3} \pi r_{s k}^{3}$ if the particle is treated as a sphere, where $m_{s k}, \rho_{s k}$ and $r_{s k}$ are the mass, density and radius of the solid particle, respectively.

\subsection{The interaction force between solid and fluid phase}

Let $\boldsymbol{f}_{j}^{S}$ be the interaction from the solid particles projected at node $j$.

$$
\boldsymbol{f}_{j}^{S}=\sum_{k=1}^{N_{s p}}\left[\nabla p\left(\boldsymbol{x}_{k}\right)+\beta_{j}\left(\boldsymbol{v}_{s}\left(\boldsymbol{x}_{k}\right)-\boldsymbol{u}_{f}\left(\boldsymbol{x}_{k}\right)\right)\right] V_{k} S_{j}\left(\boldsymbol{x}_{k}\right)
$$

The damping coefficient $\beta$ is given by Ergun's equation (Ergun 1952) in the dense region and by Wen and Yu's equation (Wen and Yu 1966) in the dilute region, as follows 


$$
\beta_{k}=\left\{\begin{array}{c}
\frac{\eta(1-\theta)}{d_{s k}{ }^{2} \theta}[150(1-\theta)+1.75 R e](\theta \leq 0.8) \\
\frac{3}{4} C_{D} \frac{\eta(1-\theta)}{d_{s k}{ }^{2}} \theta^{-2.7} R e \quad(\theta>0.8)
\end{array} .\right.
$$

where $d_{s k}$ is the diameter of a solid particle $k$, and $C_{D}$ is the drag coefficient and obtained using following expression

$$
C_{D}=\left\{\begin{array}{c}
24\left(1+0.15 R e^{0.687}\right) / R e \quad(R e \leq 1000) \\
0.43 \quad(\operatorname{Re}>1000)
\end{array} .\right.
$$

where

$$
R e=\frac{\left|v_{s}\left(x_{k}\right)-u_{f}\left(x_{k}\right)\right| \theta \rho_{f} d_{s k}}{\eta}
$$

A dimensionless parameter is advisable so that the viscosity and the particles size are removed from (8-27). In this way, a new parameter is defined

$$
\beta_{k}^{\prime}=\left\{\begin{array}{c}
\frac{(1-\theta)}{\theta}[150(1-\theta)+1.75 R e](\theta \leq 0.8) \\
\frac{3}{4} C_{D}(1-\theta) \theta^{-2.7} \operatorname{Re} \quad(\theta>0.8)
\end{array}\right.
$$

Accordingly, equation (8-26) is rewritten as

$$
\boldsymbol{f}_{j}^{S}=\sum_{k=1}^{N_{s p}}\left[\nabla p\left(\boldsymbol{x}_{k}\right)+\frac{\eta}{d_{s k}^{2}} \beta_{k}^{\prime}\left(\boldsymbol{v}_{s}\left(\boldsymbol{x}_{k}\right)-\boldsymbol{u}_{f}\left(\boldsymbol{x}_{k}\right)\right)\right] V_{k} S_{j}\left(\boldsymbol{x}_{k}\right) .
$$

For sphere solid particles, $V_{k}=\frac{\pi}{6} d_{s k}^{3}$

$$
\boldsymbol{f}_{j}^{S}=\sum_{k=1}^{N_{s p}}\left[\nabla p\left(\boldsymbol{x}_{k}\right) V_{k}+\frac{\pi d_{s k}}{6} \eta \beta_{k}^{\prime}\left(\boldsymbol{v}_{s}\left(\boldsymbol{x}_{k}\right)-\boldsymbol{u}_{f}\left(\boldsymbol{x}_{k}\right)\right)\right] S_{j}\left(\boldsymbol{x}_{k}\right) .
$$

\subsection{Numerical solution for the momentum equation}

Summing up all individual forces, equation (8-2) can be rewritten as

$$
\sum_{j=1}^{N} h_{j}(t) \frac{d \overline{m u}_{f j}}{d t}=\sum_{j=1}^{N} h_{j}(t)\left(M_{p j} \boldsymbol{g}+\boldsymbol{f}_{j}^{b c}+\boldsymbol{f}_{j}^{i n t}+\boldsymbol{f}_{j}^{s}\right) .
$$


Since $\sum_{j=1}^{N} h_{j}(t)$ is arbitrary, we have

$\frac{d \overline{m u}_{f j}}{d t}=M_{p j} \boldsymbol{g}+\boldsymbol{f}_{j}^{b c}+\boldsymbol{f}_{j}^{i n t}+\boldsymbol{f}_{j}^{s}=\boldsymbol{f}_{j}$

Given $\overline{\boldsymbol{m u}}_{f j}{ }^{n+1}$ and $\overline{\boldsymbol{m u}}_{f j}{ }^{n}$ the moment at two consecutive time steps, we have

$$
\overline{m \boldsymbol{u}}_{f j}{ }^{n+1} \approx \overline{m \boldsymbol{u}}_{f j}{ }^{n}+\Delta t \boldsymbol{f}_{j}{ }^{n}
$$

Eq. (8-35) is the MPM formula used for solving the momentum equation (7-14). It is a general form and has been applied to solve momentum equation in many applications (e.g. (Sulsky, Chen et al. 1994), (Jiang, Chen et al. 2015)). To obtain convergent solutions, the time step must follow the stability condition. It is recommended to use time step that is one tenth of the period in which sound wave passes through the cell. Artificial lower sound wave speed (artificial lower density of the fluid) may be explored to achieve high computational efficiency in practice.

Once the new momentum at the nodes becomes known, the velocity at the nodes can be updated as

$$
\boldsymbol{u}_{f j}^{n+1}=\frac{\overline{m \boldsymbol{u}}_{f j}{ }^{n+1}}{M_{j}}
$$

Then the fluid material point velocity is updated as

$$
\boldsymbol{u}_{f}^{n+1}=\sum_{j=1}^{N_{n}} \boldsymbol{u}_{f j}^{n+1} S_{j}\left(\boldsymbol{x}_{p}^{t}\right) .
$$

The nodal acceleration is updated as

$$
\boldsymbol{a}_{j}^{n+1}=\frac{\boldsymbol{f}_{j}^{n+1}}{M_{j}},
$$

and the acceleration of the fluid particle is updated as

$$
\boldsymbol{a}_{p}^{n+1}=\sum_{j=1}^{N_{n}} \boldsymbol{a}_{j}^{n+1} S_{j}\left(\boldsymbol{x}_{p}^{t}\right) .
$$


The strain rate tensor, stress tensor will be updated based on the fluid velocity $\overline{\boldsymbol{u}}_{f}{ }^{n+1}$, which is calculated from the fluid particle acceleration,

$$
\overline{\boldsymbol{u}}_{f}^{n+1}=\boldsymbol{u}_{f}^{n}+\boldsymbol{a}_{p}^{n+1} \Delta t
$$

The updated position of the fluid particle is calculated as

$$
\boldsymbol{x}_{f}^{n+1}=\boldsymbol{x}_{f}^{n}+\boldsymbol{u}_{f}^{n+1} \Delta t
$$

Note, the operation for updating the fluid particle coordinate will not be done until the calculation of interaction force (contact force) for the solid particle is finished, because the position should be at the previous time step in order to calculate the forces for both fluid particles and solid particles based upon the known positions. This algorithm is called modified update stress last (MUSL) (Buzzi, Pedroso et al. 2008). 


\section{Chapter 9. Convergence Study}

There exists fundamental difference between discrete solid particles and the computational particles used in the MPM. The discrete solid particles, as are observed in granular materials, are physical particles and are represented in DEM with their actual size or representative size. The size of computational particles are not physical particles. They are used to represent parts of a continuum. Within the MPM, as with several other particle-based methods, a computational particle is best viewed as a representative volume element (RVE) rather than an actual particle (or grain) in a granular medium. Because of this, the size of a computational particle can vary.

The size of computational particle directly affects the accuracy and efficiency of a particlebased numerical method. For a correct numerical method, fine elements or smaller sized computational particles generally lead to high accuracy, in other word, the solution converges as the size of computational particles decreases. This convergence does not prove a numerical method, but it is a necessary condition to verify a new method.

Convergence study generally compares numerical results of varying size of elements (particles) with analytical solutions. Analytical solutions in fluid dynamics are rare if they exist.

Here we compare numerical solutions with a group of data from a physical experiment presented in the research paper by Sun et al. (Sun, Sakai et al. 2013). For the readers' convenience, a brief description is given below.

In this experiment, the mixture of water and glass beads are set in a rectangular tank with an altitude of $0.1 \mathrm{~m}$ (shown in Figure 9.1). The mass of glass beads is 200 gram and the density is $2500 \mathrm{~kg} / \mathrm{m}^{3}$. The diameter of the beads is $2.7 \mathrm{~mm}$. The dimensions of the tank are $0.2 \mathrm{~m}$ in length, $0.1 \mathrm{~m}$ in width and $0.15 \mathrm{~m}$ in altitude. The mixture is contained in the left side with a gate being 
set at $0.05 \mathrm{~m}$ from the left side wall of the tank. Time starts at the moment when the gate is raised at a constant rate of $0.68 \mathrm{~m} / \mathrm{s}$.

In our numerical model, the stiffness, restitution coefficient and friction coefficient of the solid particles are chosen as $1000 \mathrm{~N} / \mathrm{m}, 0.9$ and 0.2 , respectively. Non-slip boundary condition is applied where water contacts the walls and the bottom of the tank. A fixed time increment of $1.0 \times 10^{-5} \mathrm{~s}$ is used throughout the simulation with an assumed water bulk modulus $2.2 \times 10^{7} \mathrm{~Pa}$. The density, viscosity of water are taken as $1000 \mathrm{~kg} / \mathrm{m}^{3}, 0.001$ Pas respectively. In the MPM there are two parameters that determine the size of a computational particle: one is the size of the MPM cell and another is the number of particles per cell. For the size of the MPM cell, a group of numerical tests with cell size $10 \mathrm{~mm}, 8 \mathrm{~mm}, 6.25 \mathrm{~mm}, 5.0 \mathrm{~mm}$, and $4.1 \mathrm{~mm}$ are conducted. Because the length of the tank is $200 \mathrm{~mm}$, the corresponding number of cell in this direction is $20,24,32,40$ and 48 respectively. The multiples of 4 are chosen simply because there is a gate at $1 / 4$ of the tank length. The second group of tests are the same, but the number of particles per cell varies. In the convergence study, the model is named with these two parameters. Take model Grid40_8p as an example. 40 represents 40 cells in length direction and 8 p shows there are 8 particles per cell.

In the physical experiment, both water and glass beads moves in the same direction but at different velocity. The motion of the front lines for both water and glass beads are recorded by high-speed camera and measured thereafter. Four snapshots are taken to show the motion of the solid phase and the flow of the fluid in Figure 9.2.

The results from model Grid40_8p are shown in Figure 9.3. Visual comparison provides first step to check if the numerical solution makes correct prediction. Since there is no other way to find a better solution, the observed motion from the physical experiment serves as a reference. We 
find that the numerical solution from Grid40_8p is very close to the observational data. Now it is a goal to know whether models with different parameters provide similar prediction.

\subsection{The effect of the MPM cell size}

The MPM cell size determines how accurately the interpolation reflects the real situation. If all particles are processed in one cell, we can only get one averaged value, which will be far from the accuracy requirement. Though fine computation particles are desirable for improving accuracy, there is a limit from the size of solid particles. Since this combined DEM-MPM method requires more solid particles in a cell, the MPM cell cannot be smaller than the size of solid particles. The diameter of glass beads is $2.7 \mathrm{~mm}$ and the tank is $200 \mathrm{~mm}$ long, which produces the maximum possible cell number in this direction (74 cells). If 2 solid particles are aligned in this direction, the cell number will be 37 at most.

In order to demonstrate the effect of the MPM cell size on the accuracy of numerical modeling, we designed a group of models with cell size varying from $4 \sim 10 \mathrm{~mm}$. The prediction from these models are shown in Figure 9.4. It is observed that models with coarse cell predict slow fluid motion. The model with smallest cell size $(4.1 \mathrm{~mm})$, i.e., the model Grid48_8p predicts the highest fluid motion. The fluid takes less than 0.2 second to reach the opposite wall. The model with grid cell size of $10 \mathrm{~mm}$ predicts the fluid takes 0.3 second to reach the opposite wall. The difference between model Grid48_8p and Grid40_8p is significantly smaller than the difference between model Grid24_8p and Grid20_8p, which indicates the numerical solutions converge. If the solution from model Grid48_8p is regarded as a reference, we can see the convergence tendency as the grid mesh becomes fine. 
The motion of the solid particles predicted from this group of models shows the same tendency. In Figure 9.5, it is observed that slow motion predicted in the model of coarse meshes. Models of fine meshes predict fast motion. Are models of fine meshes more correct? This question will be answered in detail in next section. Here, we simply confirm that the model with the finest meshes predicted motion of both phases comparable with that from the physical experiment of the same setting. Therefore, Figure 9.5 shows that the error decreases as the cell size gets smaller.

\subsection{The effect of the number of particles per cell}

A material point, carrying all material information like mass, momentum, strain and stress, is a point mathematically with no shape factor. The collection of motion of material particles reflects the motion and deformation of the studied continuum or a collection of discrete particles. With only one particle in a cell, this material point carries information of an averaged motion of material around its mass center. If there is one spherical solid particle in a cubic cell and located at the center, the rest space will be filled with fluid. If only one material particle (point) is designed to represent all fluid in this cell, this material particle must overlap with the solid particle. If 8 material particles are used, they could be located near corners without overlapping. After interaction with solid particles due to viscous drag, fluid particles at different location will have different velocity. More particles per cell should improve the representation of continuum, thus making a prediction of high accuracy. The proposed new approach is expected to act this way. After a series of numerical tests, we confirm that the new approach does perform this way.

The motion of the fluid predicted in this series of test is shown in Figure 9.6. It is observed that models of fewer particles predict either motion that is too slow (as that from model Grid40_1p) or too fast (as that from model Grid40_2p). Very close curves are predicted from models of more particles per cell (Models Grid40_4p and Grid40_8p). 
The motion of the solid particles predicted in this series is shown in Figure 9.7. The numerical predicts show the same tendency. This confirms that our new algorithm does predict more accurately as the number of particles per cell increases. It should be noted, however, that an accurate prediction due to increasing the number of particles per cell comes with cost in computational time because of more particles. As 8 particles per cell give a quite good prediction, models with more than 8 particles per cell is no longer needed.

We also noticed that the convergence tendency in the prediction of solid phase is somewhat different from that for fluid phase. The prediction for the fluid phase seems to be converging gradually, whereas the prediction for the solid phase in the models with more than 1 particle per cell are very close to each other, and far from the model with only one particle per cell. Though the cause is unclear, this tendency shows that good results can be achieved using half of the ideal number of particles per cell, which is thought to be $2 \times 2 \times 2=8$ in a three-dimensional cell, thus significantly reducing computing time without losing much accuracy.

In summary, the convergence study shows the new method has the expected performance on solution convergence. 
Table 9.1 Physical properties and computational parameters.

\begin{tabular}{ll}
\hline Item & value \\
\hline Solid phase & 7762 \\
\hline DEM particle number & 0.0027 \\
\hline Particle diameter (m) & $2.5 \mathrm{e} 3$ \\
\hline Density (kg/m3) & $1.0 \mathrm{e} 3$ \\
\hline Stiffness (N/m) & 0.9 \\
\hline Restitution coefficient & 0.2 \\
\hline Friction coefficient & \\
\hline Liquid phase & 26,879 \\
\hline MPM particle number & 0.005 \\
\hline MPM mesh cell size (m) & $2 \times 2 \times 2$ \\
\hline Initial Number of particle per cell & 1000 \\
\hline Density (kg/m $\mathbf{m}^{3}$ & 0.001 \\
\hline Dynamic viscosity (Pas) & $2.2 \mathrm{e} 7$ \\
\hline Virtual bulk modulus (Pa) & \\
\hline Solid structural boundary & $0.2 \times 0.1 \times 0.15$ \\
\hline Dimensions (m) & 22 \\
\hline Number of triangle meshes & 0.68 \\
\hline Gate rising rate (m/s) & \\
\hline
\end{tabular}



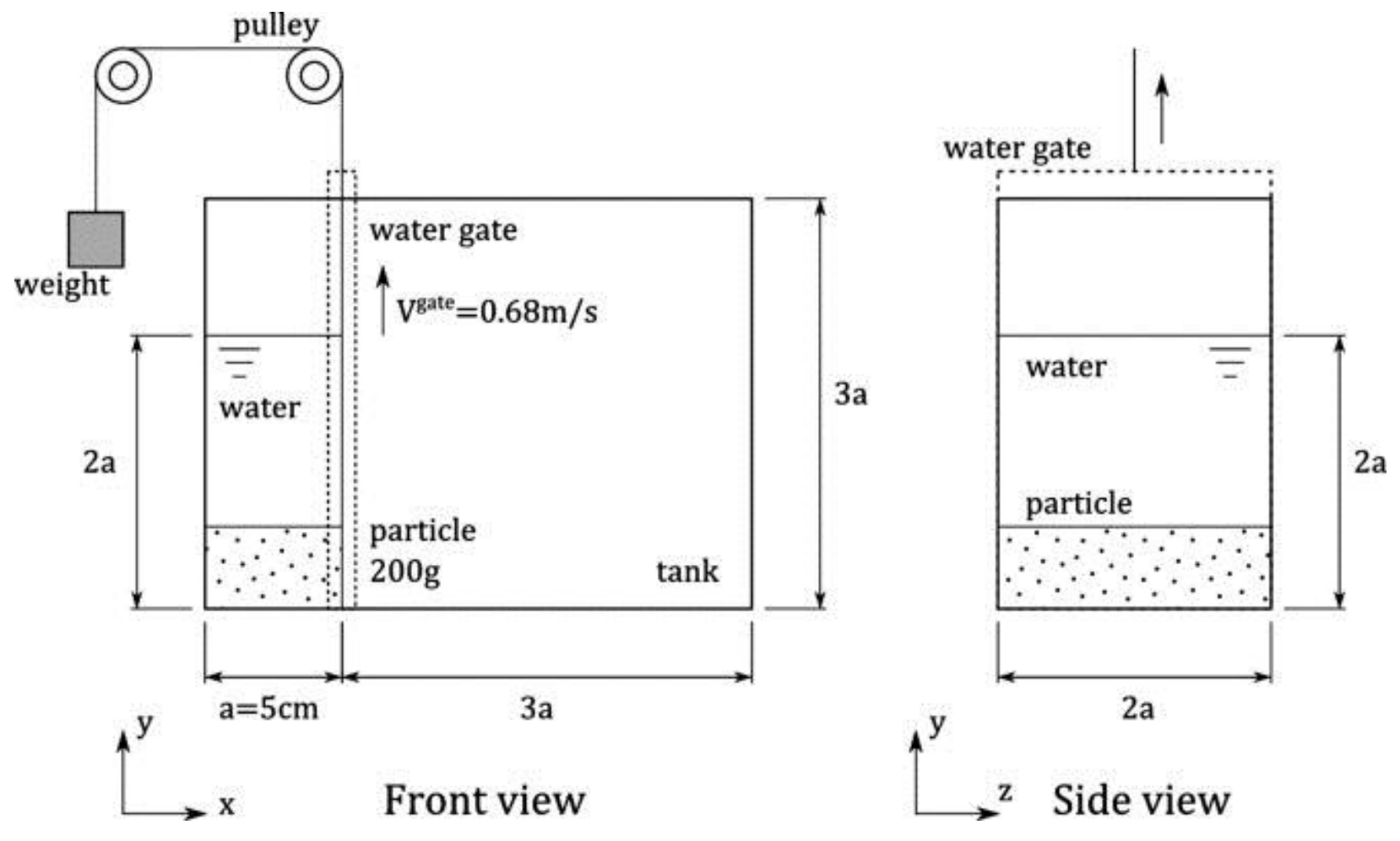

Figure 9.1 Setting up of the dam-break test for solid-liquid mixture.

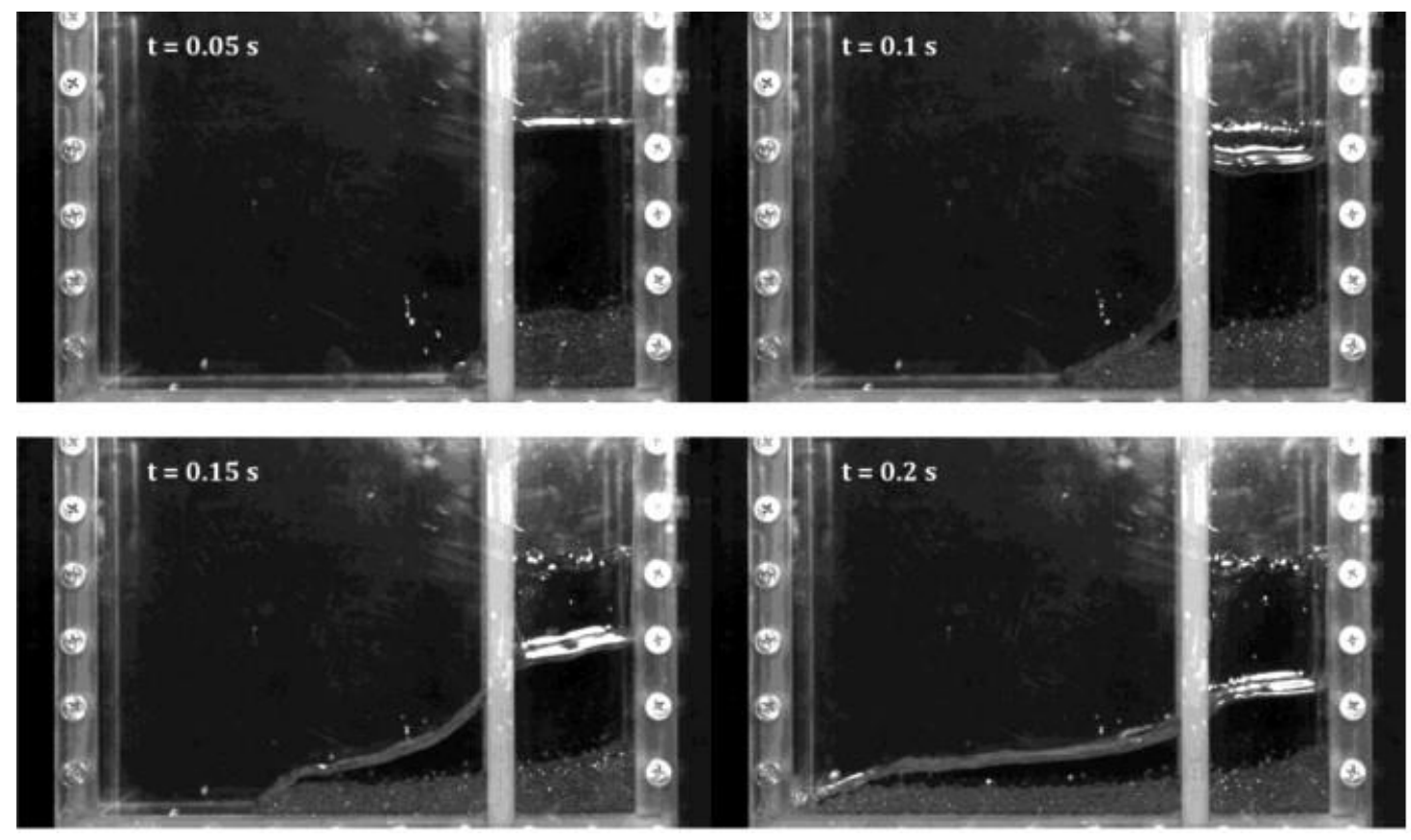

Figure 9.2 Snapshots showing the motion of solid-liquid mixture in the physical test. 

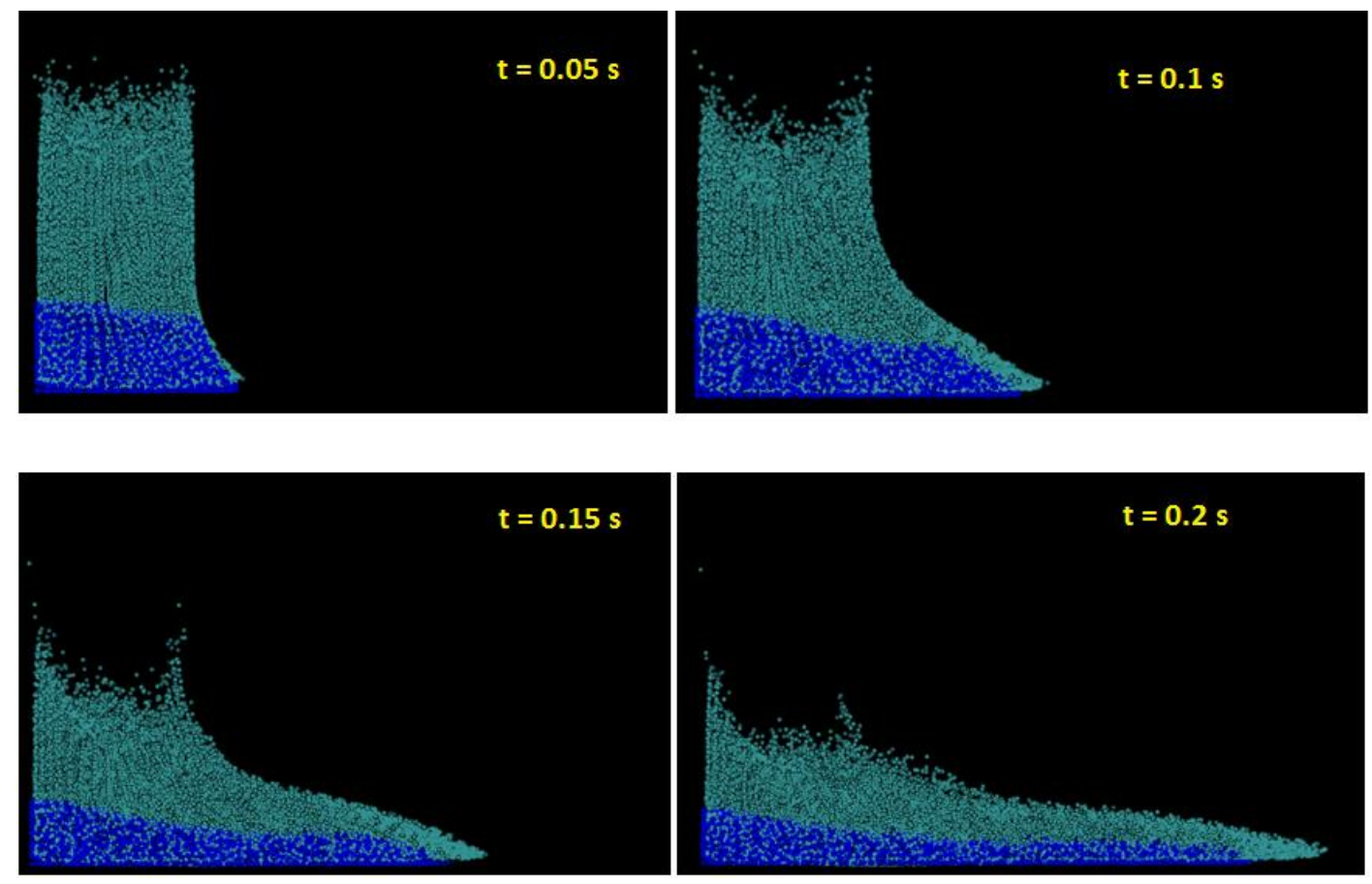

Figure 9.3 Simulation of two-phase flow in a tank, 4 snapshots from numerical modeling. The dark blue dots stand for the glass beads, while light blue dots for water. This figure is prepared by using VMD (Humphrey, Dalke et al. 1996). 


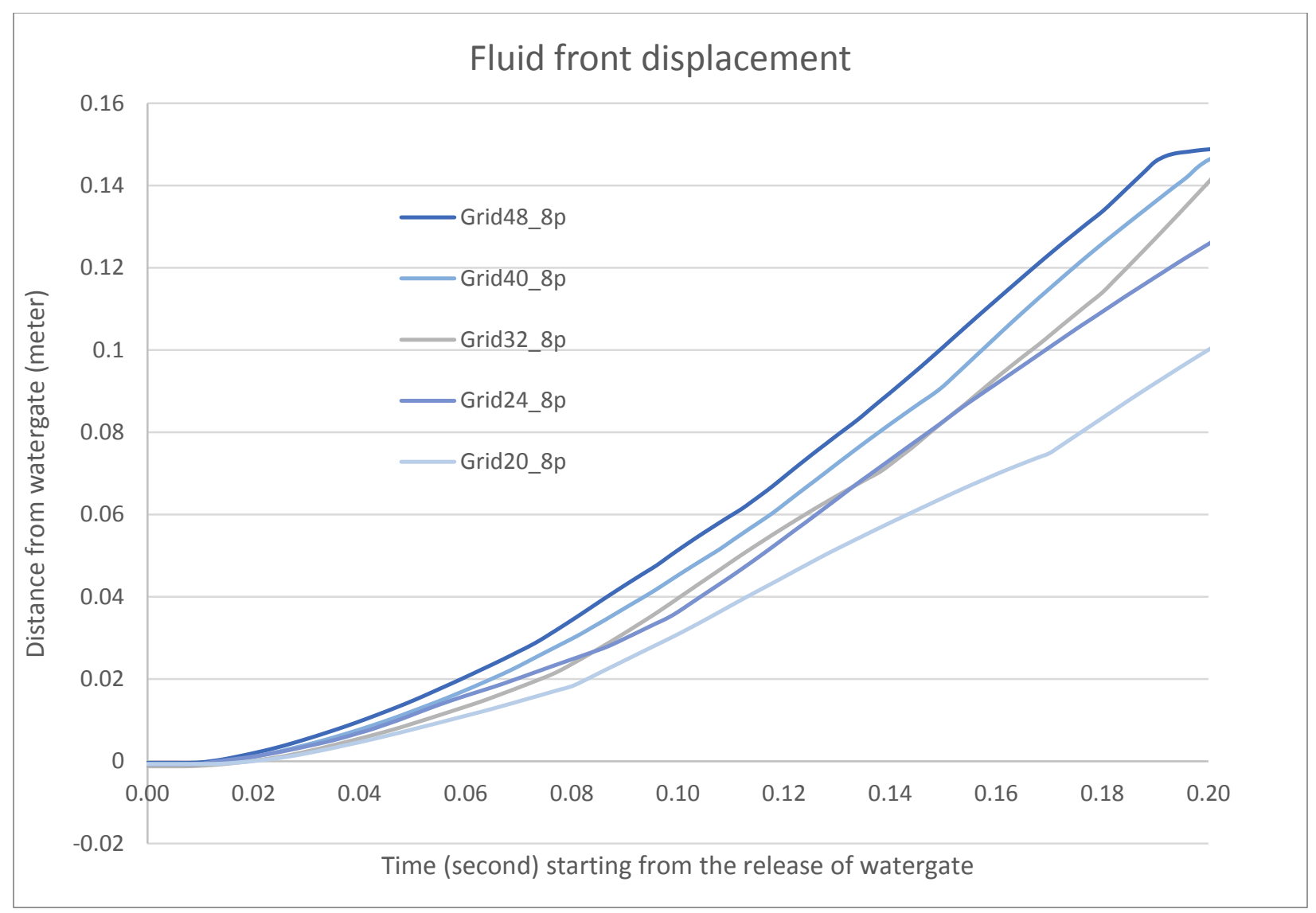

Figure 9.4 Effect of the MPM cell size on the numerical solution for the fluid phase. 


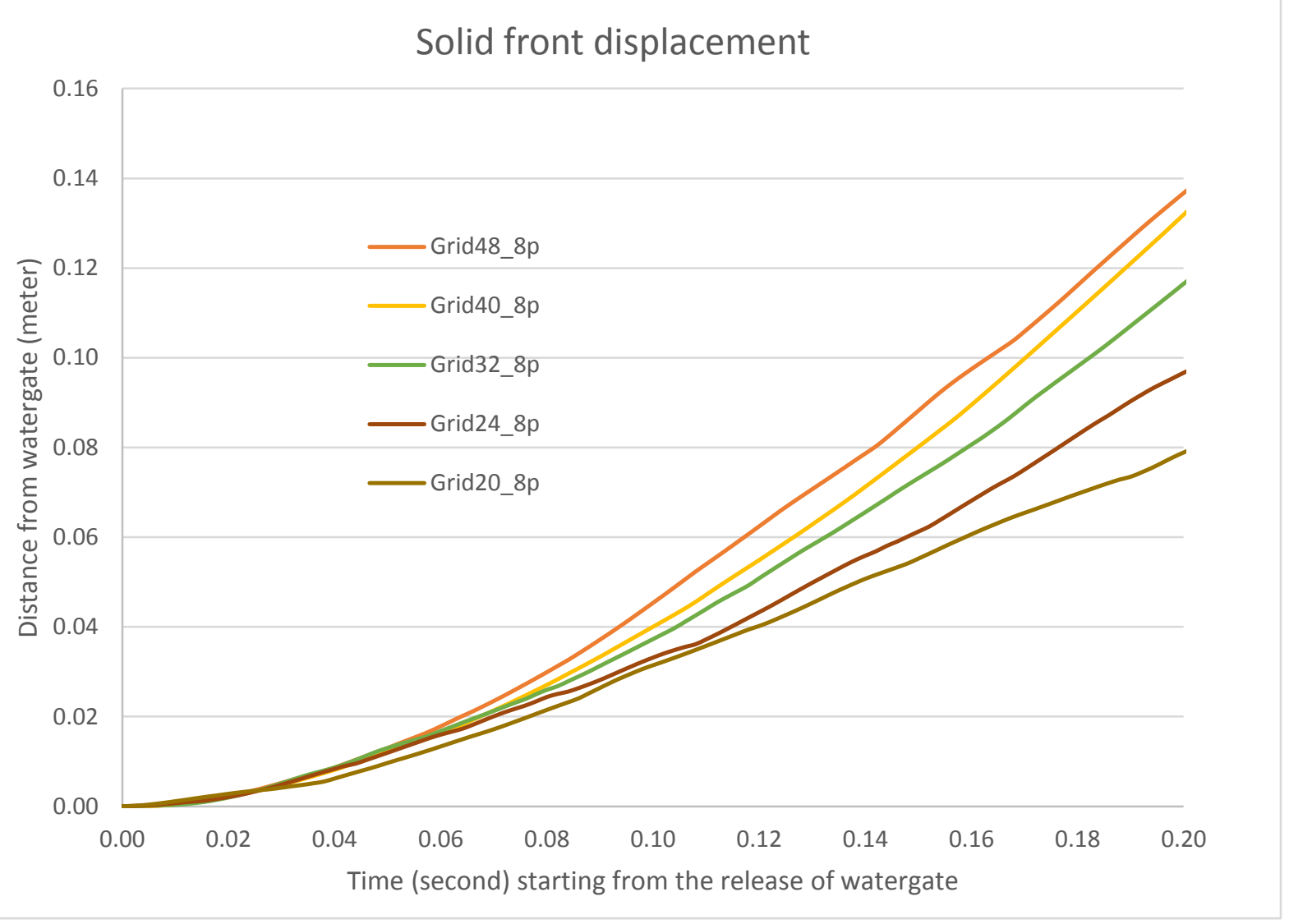

Figure 9.5 Effect of the MPM cell size on the numerical solution for the solid phase. 


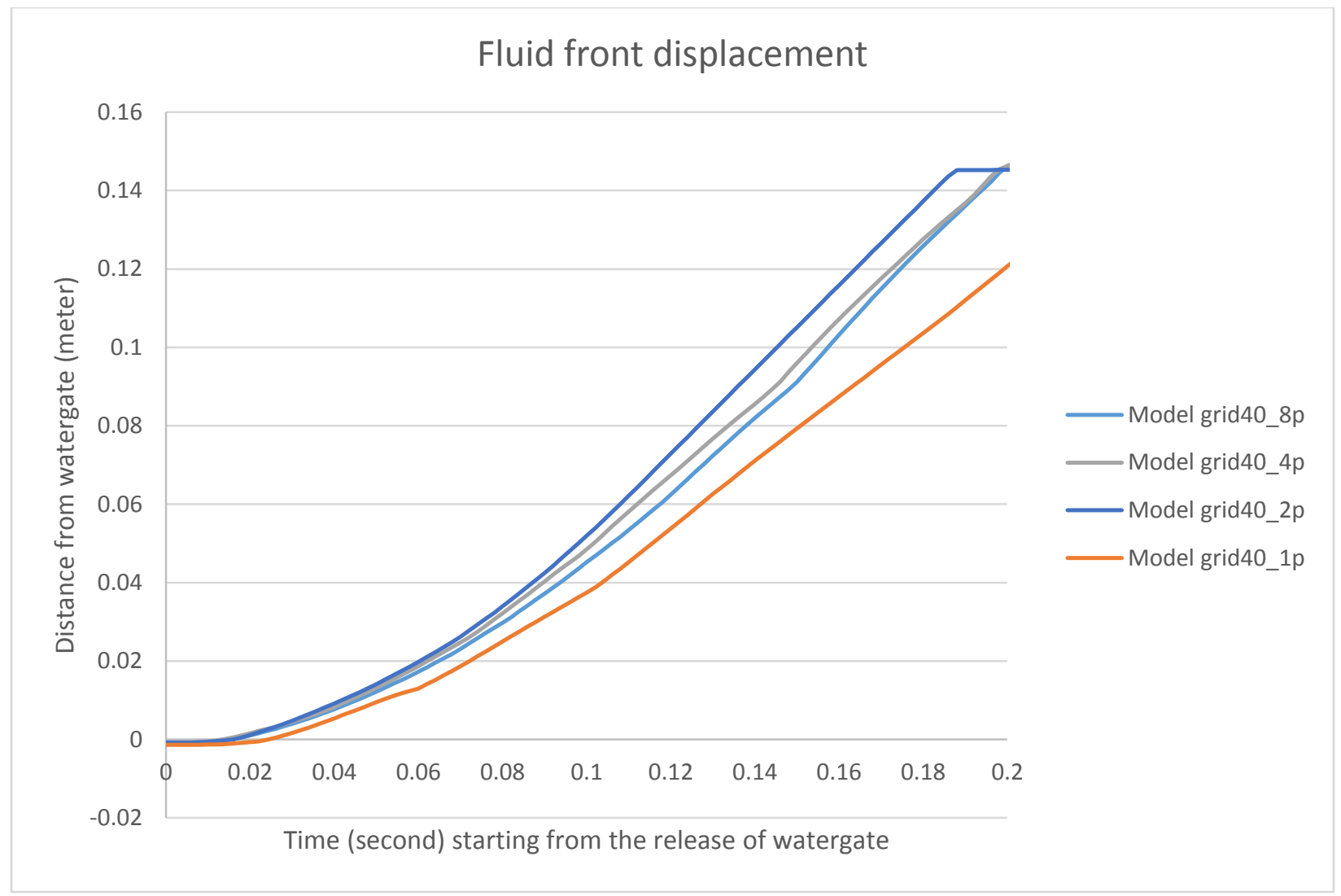

Figure 9.6 Effect of the number of MPM particles per cell on the numerical solution for the fluid motion. 


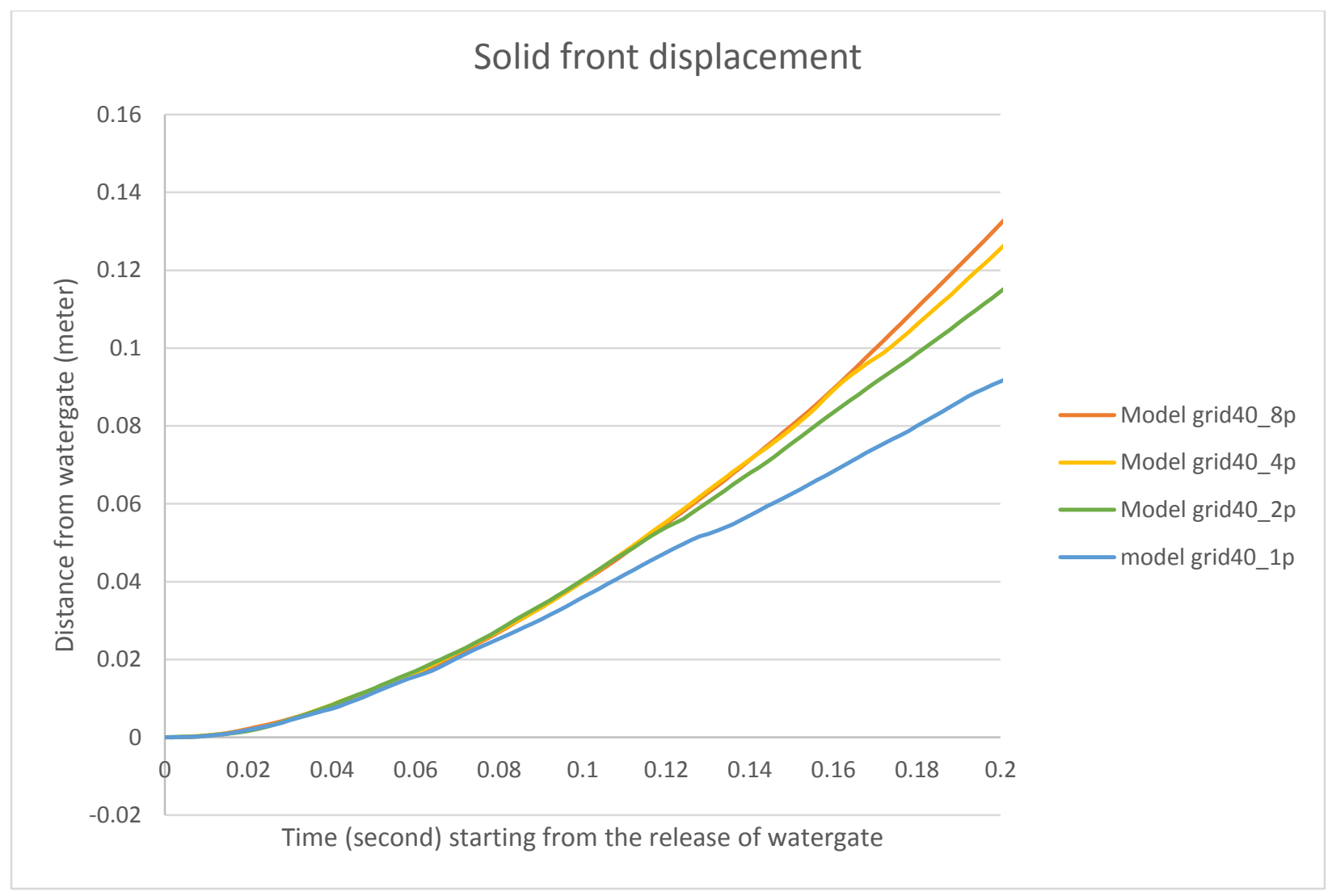

Figure 9.7 Effect of the number of MPM particles per cell on the numerical solution for the solid motion. 


\section{Chapter 10. Verification and Validation}

In order to accurately predict a process using numerical methods, a new code must pass verification and validation. Code verification is a prerequisite to code validation. The former one requires that the code is correct, mathematically. If there exists an analytic solution, the code should be able to produce a numerical result that approximates to the analytic solution in a certain convergence rate. The latter one requires that the code can reproduce the numerical result that is close to the result of physical experiments. Once the code passes the validation, the confidence to predict the real world events is then established.

Though there are many issues around the DEM, the confidence in using DEM to simulate multi-body motion is widely established in industries (Zhu, Zhou et al. 2007). Thus, we will not address the verification and validation of the DEM in this paper, instead, we focus our attention on the verification and validation of the MPM in the simulation of multiphase fluid motion. So far, the analytical solution of a multiphase flow problem has not been found. Our stratagem of code verification is to try many different cases and check that the model predictions are correct in mathematics and in physics. For example, when the volume fraction is assumed to be $100 \%$, the multiphase model actually becomes a single phase model, therefore, the new code should be able to reproduce the results of a single phase experiment. Models of varying size of background mesh put the MPM-DEM on the convergence test. These tests are not a sufficient condition to approve the code, but a very useful step in the code development. Further validation is completed in another test in which both solid phase and fluid phase are presented. 


\subsection{Water flow after reservoir dam breakage}

First, we compare our result with that obtained by Mast, Mackenzie-Helnwein et al. (Mast, Mackenzie-Helnwein et al. 2012) simply because we adopt similar stratagem and there are experimental data available in (Martin and Moyce 1952).

A simplified dam break is sketched in Figure 10.1. A water column of initial height, $h_{0}$, and length, $l_{0}$, is initially constrained horizontally and rests on a smooth, flat surface. The dynamic code is run about 0.3 second to allow the static pressure field being stabilized. It is now necessary to mark the time $t=0$ when the gate on the right-hand side is removed and the water is allowed to flow freely under the force of gravity. The following parameters describe the free surface run out:

$$
T=t \sqrt{\frac{h_{0} g}{l_{0}^{2}}}, L(T)=\frac{l(T)}{l_{0}}, \text { and } H(T)=\frac{h(T)}{h_{0}},
$$

where $T, L(T)$ and $H(T)$ are the dimensionless time, length, and height ratios, respectively. The parameters $h_{0}$ and $l_{0}$ are 4.0 meters each. The term $g=|g|=9.8 \mathrm{~m} / \mathrm{s}^{2}$. The viscosity of water is taken as 0.001 Pas. There are $2 \times 2 \times 1$ particles in each cell initially. The physical experiment is basically a 2D case. Our numerical model takes a thin slice in 3D space of 4 meter high and 24 meter long. The thickness of this slice is the size of a fluid particle. The initial configuration of the water column is of square shape with length of 4 meters, which can be divided into regular meshes. The rest domain is divided into regular meshes of the same size. The cell resolution is 0.125 meter in length, 0.125 meter in height and 0.0625 meter in thickness for model MPM32. Table 1 summarizes the parameters used in the numerical tests.

The predictions from the MPM is shown in figure 12.2 by a series of snaps taken at time $T=$ $0,0.5,1.0,1.5,2.0,2.5,3.0$. It is observed that water surface tilting and the right corner flowing 
out. The water flow predicted from the MPM model is comparable with that by Mast, MackenzieHelnwein et al. (Mast, Mackenzie-Helnwein et al. 2012).

The quantitative comparison between the model predictions and experimental data are shown in Figure 10.3 and 10.4. In Figure 10.3, the predictions from a MPM model with free-slip and nonslip boundary conditions are given, respectively. The former one is marked as MPM1 and the latter one as MPM2. The MPM1 predicts the result which is identical to that by Mast, MackenzieHelnwein et al. (Mast, Mackenzie-Helnwein et al. 2012). This is understandable because we use the cell-based anti-locking technique as they did. Note that the predicted length is larger than the length from the experimental data. The MPM2 gives a much better fit to the experimental data. It is reasonable that free-slip boundary condition promotes the flow, while non-slip boundary condition represents the physical condition better.

The resolution of the MPM grid is another factor that may affect the model predictions. Figure 10.3B shows the predictions from three MPM models. There are 16 cells in the length of initial length $4.0 \mathrm{~m}$ in the model MPM16, and 24 in MPM24, and 32 in MPM32. It is very clear from Figure 12.3B that the prediction becomes better and better as the spatial resolution improves.

The height history predicted from these models is also shown in Figure 10.4 (A and B). Generally, they are comparable with the experimental data. Larger error occurs at the $\mathrm{T}>2.5$ when the water layer becomes very thin (see Figure 10.2).

Both visual and quantitative comparisons show that the cell-based implementations in our code are successful, but this only demonstrates that our code can be used for the simulation of pure fluid case. We need to demonstrate more examples to show the success of our method in the multiphase flow. 


\subsection{Motion of glass beads and water in a tank}

Here we demonstrate a case very similar to the water dame breakage, however, this time there is a solid phase--glass beads mingled with water inside the rectangular tank. This case is presented in the research paper by Sun et al. (Sun, Sakai et al. 2013). For the readers' convenience, a brief description is given below.

The mixture of water and glass beads has an altitude of $0.1 \mathrm{~m}$. The mass of glass beads is 200 gram and the density is $2500 \mathrm{~kg} / \mathrm{m}^{3}$. The diameter of the beads is $2.7 \mathrm{~mm}$. The dimensions of the tank are $0.2 \mathrm{~m}$ in length, $0.1 \mathrm{~m}$ in width and $0.15 \mathrm{~m}$ in altitude. The mixture is contained in the left side with the gate being set at $0.05 \mathrm{~m}$ from the left side wall of the tank. Time starts at the moment when the gate is raised at a rate of $0.68 \mathrm{~m} / \mathrm{s}$. The stiffness, restitution coefficient, and friction coefficient of the solid particles are chosen as $1000 \mathrm{~N} / \mathrm{m}, 0.9$ and 0.2 , respectively, same as those used in SPH-DEM simulation by Sun et al. (Sun, Sakai et al. 2013). Non-slip boundary condition is applied where water contacts the walls and the bottom of the tank. A fixed time increment of $1.0 \times 10^{-5} \mathrm{~s}$ is used throughout the simulation. We simulate water with 26879 material particles and the background mesh of $0.005 \mathrm{~m}$ resolution. The physical and model parameters are listed in Table 9.1.

We first prepare settled configuration of the mixture before the gate being raised, and then simulate the process of two-phase flow. Figure 10.5 shows four snapshots of the result from the simulation, for selected time instants of $\mathrm{t}=0.05,0.1,0.15$ and $0.2 \mathrm{~s}$. Quantitative comparison with experimental data is presented in Figure 10.6. It is observed that the solid particles fall a little bit behind the water front, consistent with the experimental data. Quantitative comparison in figure 10.6 gives us sufficient evidence that the proposed DEM-MPM method is capable of making prediction with high fidelity. 


\subsection{Solid-fluid flow in a rotating jar}

Our original idea is to validate the new method with more experimental data. The case of solidfluid flow in a rotating jar presented by Sun et al. (Sun, Sakai et al. 2013) is chosen for this purpose. Because no boundary condition is described, we simply apply non-slip condition on the wall of the jar whenever the fluid contacts. It turns out this model is unable to reproduce what it is observed in the experiment.

This model simulates the solid-fluid flow in a cylindrical jar with both inner diameter and depth at $100 \mathrm{~mm}$. The jar contains 7755 solid spherical particles (glass beads) with diameter $2.7 \mathrm{~mm}$ and density $2700 \mathrm{~kg} / \mathrm{m}^{3}$. It also contains $0.297 \mathrm{~kg}$ of water with density $1000 \mathrm{~kg} / \mathrm{m}^{3}$ and viscosity 0.001 Pas. The jar rotates at the rate of 104 revolutions per minute. The DEM is exploited to simulate the glass beads, and the stiffness, restitution coefficient and friction coefficient are set to be 1000 $\mathrm{N} / \mathrm{m}, 0.8$ and 0.3 , respectively. The MPM is for fluid phase with 15406 particles of identical fluid mass. There are $36 \times 36 \times 10$ cells evenly distributed in $100 \mathrm{~mm} \times 100 \mathrm{~mm} \times 100 \mathrm{~mm}$ cubic space with $1 \times 1 \times 4$ MPM particles in each cell when the cell is full of fluid particles at the initial condition. The interior surfaces of the jar are simulated with 1440 triangles. These parameters for the simulation are listed in Table 10.2.

Because of the constant rotating rate, the configuration of the glass beads inside the jar will take a stable shape after a certain period of transition. The configuration of the beads is measured and simulated. In the experiment, the maximum altitude of the beads in the stable state is 62.18 $\mathrm{mm}$ in average with standard deviation $1.71 \mathrm{~mm}$. In the simulation with non-slip boundary condition applied, the model predicted maximum altitude in bead bed is $67 \mathrm{~mm}$. We also noticed that the water particles diffuse over space inside the jar. There is no clear free surface observed. The comparison is shown in Figure 10.7 (A) and (B). 
What causes this difference? The difference is also noticed in the first case of pure water flow -- it is due to the difference in boundary condition. Strictly speaking, non-slip boundary condition and free-slip condition are two end-members in describing the interaction between the boundary and the interior mass. Non-slip boundary works better in the case of low Reynolds number while free-slip for the large Reynolds number. In reality, the interaction may exist between the two endmember conditions. In the case using non-slip boundary condition, the boundary has the strongest coupling with the interior mass. This is the case for rotating jar: when non-slip boundary condition is applied, more energy is transferred from boundary wall to the interior mass including glass beads and water. The lifting force is high and the final balanced altitude is higher than the real value observed in the laboratory.

A reasonable guess is that the boundary may be partially sticky (non-slip) and partially slippery (free slip). To check this hypothesis, we tested a case in which 50\% of the boundary velocity is sticky and the rest is slippery. The result is shown in Figure 10.7 (C). It is obvious that the simulation fits the experiment result much better. The water particles form a clear free surface and the maximum altitude in bead bed is $61 \mathrm{~mm}$. In the case water is full in the rotating jar, the Reynolds number could be estimated up to $2 \times 10^{7}$, much higher than the value for the laminate flow in which non-slip boundary is justified. The method of finding a proper boundary condition for high Reynolds number flow should continue to be explored. The comparison shown in this case highlights the importance of boundary condition in fidelity of numerical modeling.

One frustrating problem in the application of the material point method is that the numerical solution becomes unstable after long simulation time, like in the gear noise control problem (personal communication with Z. Chen). In the original test, the jar rotates 4-5 turns. To check if a similar problem exists in the current DEM-MPM simulation, a test of the fast spinning jar model 
runs 25 turns and the numerical result is still very stable. Just because of the large size of the output data, the VDM stops working at the $17^{\text {th }}$ turn for the animation of two phase. All 7500 frames of solid particle motion in the 25 turns are normally displayed as the first a couple of turns.

The combined MPM-DEM method developed in this study applies for the interaction among solid discrete elements and the fluid in between. Theoretically, it works for both liquid saturated or unsaturated and gas. So far we have verified it works for liquid, further verification is required for gas. The proposed MPM-DEM method is far from perfect. In this method the MPM cell is required to be larger than the size of solid particles. In an industrial application like a sieve, in which small particles are allowed to pass, but big particles (compared with sieve size) are not, the accuracy of this method and the applicability of this method is a problem. Future study in this direction is in need. 
Table 10.1 Physical properties and computational parameters.

\begin{tabular}{ll}
\hline Item & value \\
\hline Water column initial height $(\mathbf{m})$ & 4.0 \\
\hline Water column initial length $(\mathbf{m})$ & 4.0 \\
\hline MPM mesh cell size $(\mathbf{m})$ & Varying from 0.125 to 0.25 \\
\hline Initial Number of particle per cell & $2 \times 2 \times 1$ \\
\hline Density $\left(\mathbf{k g} / \mathbf{m}^{\mathbf{3}}\right)$ & 1000 \\
\hline Bulk modulus $($ Pa) & $2.2 \mathrm{e} 9$ \\
\hline Dynamic viscosity (Pas) & 0.001 \\
\hline Time step (s) & $1.0 \mathrm{e}-5$ \\
\hline
\end{tabular}

Table 10.2 Physical properties and computational parameters.

\begin{tabular}{ll}
\hline Item & value \\
\hline Solid phase & \\
\hline DEM particle number & 7755 \\
\hline Particle diameter $(\mathrm{m})$ & $2.7 \mathrm{e} \_3$ \\
\hline Density $(\mathrm{kg} / \mathrm{m} 3)$ & $2.5 \mathrm{e} 3$ \\
\hline Stiffness $(\mathrm{N} / \mathrm{m})$ & $1.0 \mathrm{e} 3$ \\
\hline Restitution coefficient & 0.9 \\
\hline Friction coefficient & 0.3 \\
\hline Liquid phase & \\
\hline MPM particle number & 15,406 \\
\hline MPM mesh cell size $(\mathrm{m})$ & 0.00278 \\
\hline Initial Number of particle per cell & $2 \times 2 \times 2$ \\
\hline Density (kg/m $\left.{ }^{3}\right)$ & 1000 \\
\hline Dynamic viscosity (Pas) & 0.001 \\
\hline Virtual bulk modulus (Pa) & $2.2 \mathrm{e} 7$ \\
\hline Solid structural boundary & \\
\hline Number of triangle meshes & 1440 \\
\hline Rotation speed (rpm) & 104 \\
\hline
\end{tabular}



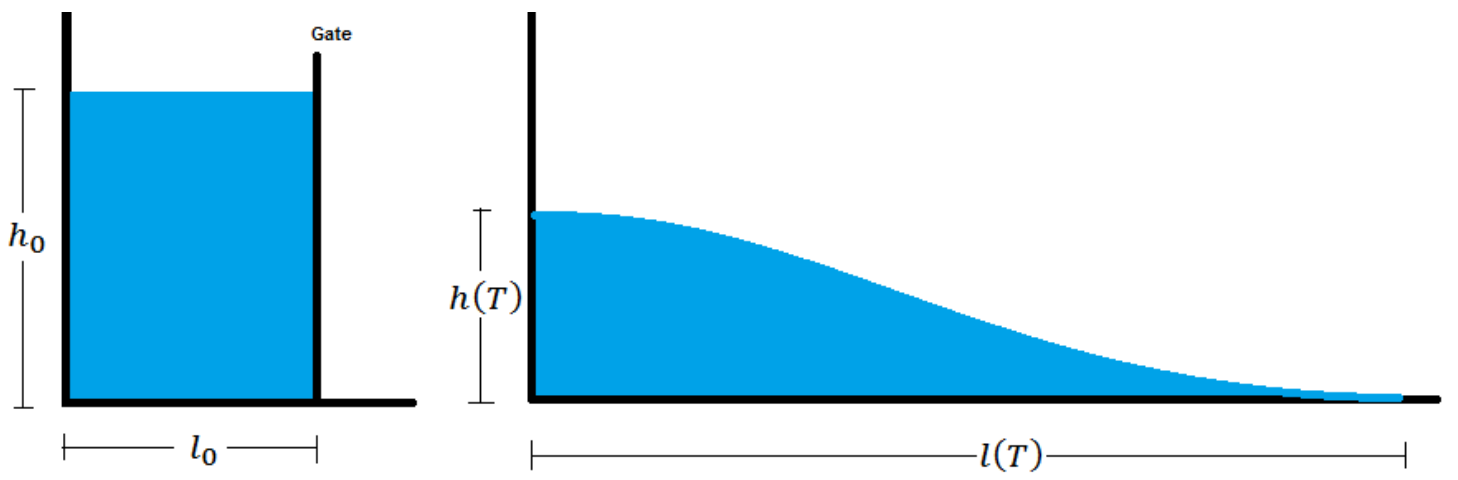

Figure 10.1 Dam breakage simulation.

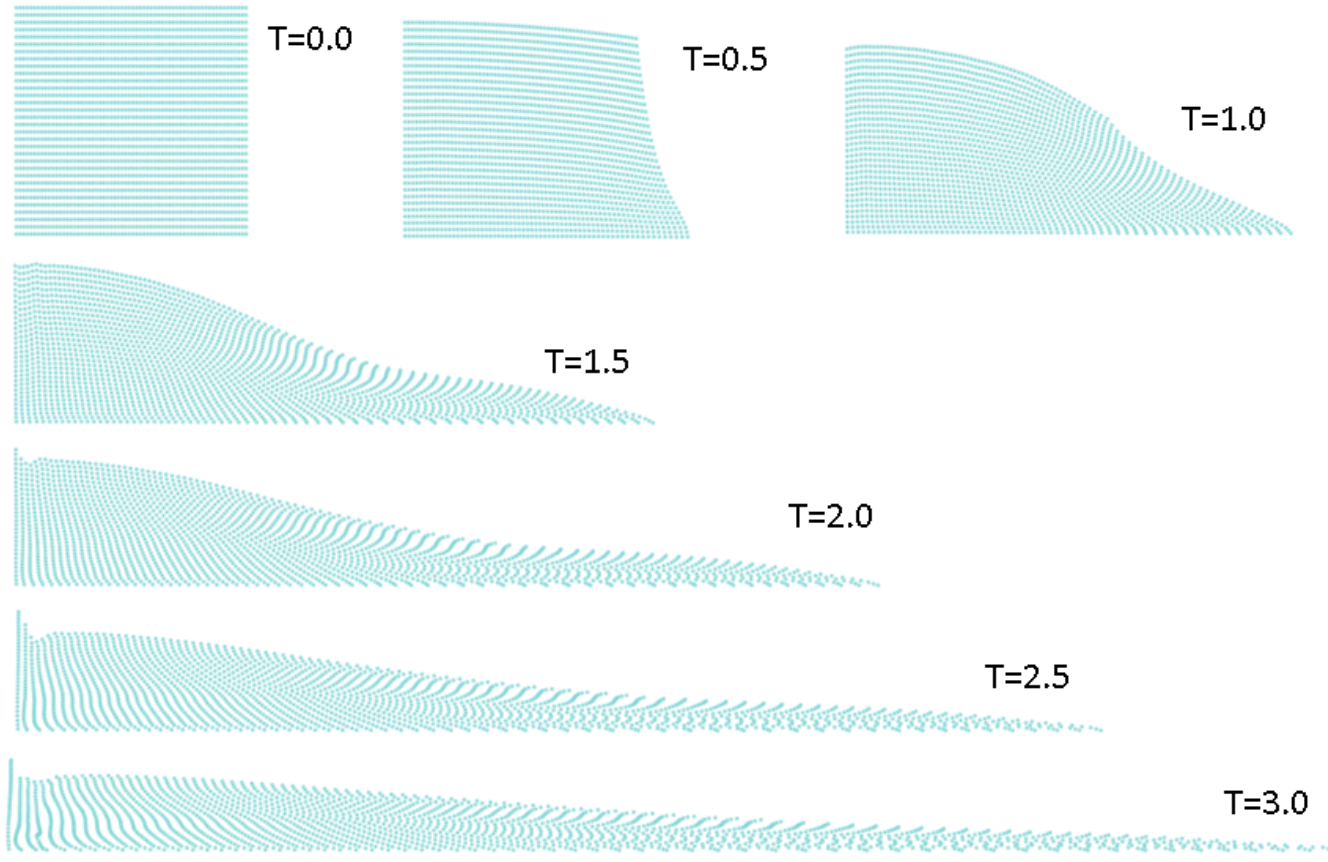

Figure 10.2 Dam breakage sequence predicted from a MPM simulation. This figure is prepared by using VMD (Humphrey, Dalke et al. 1996). 

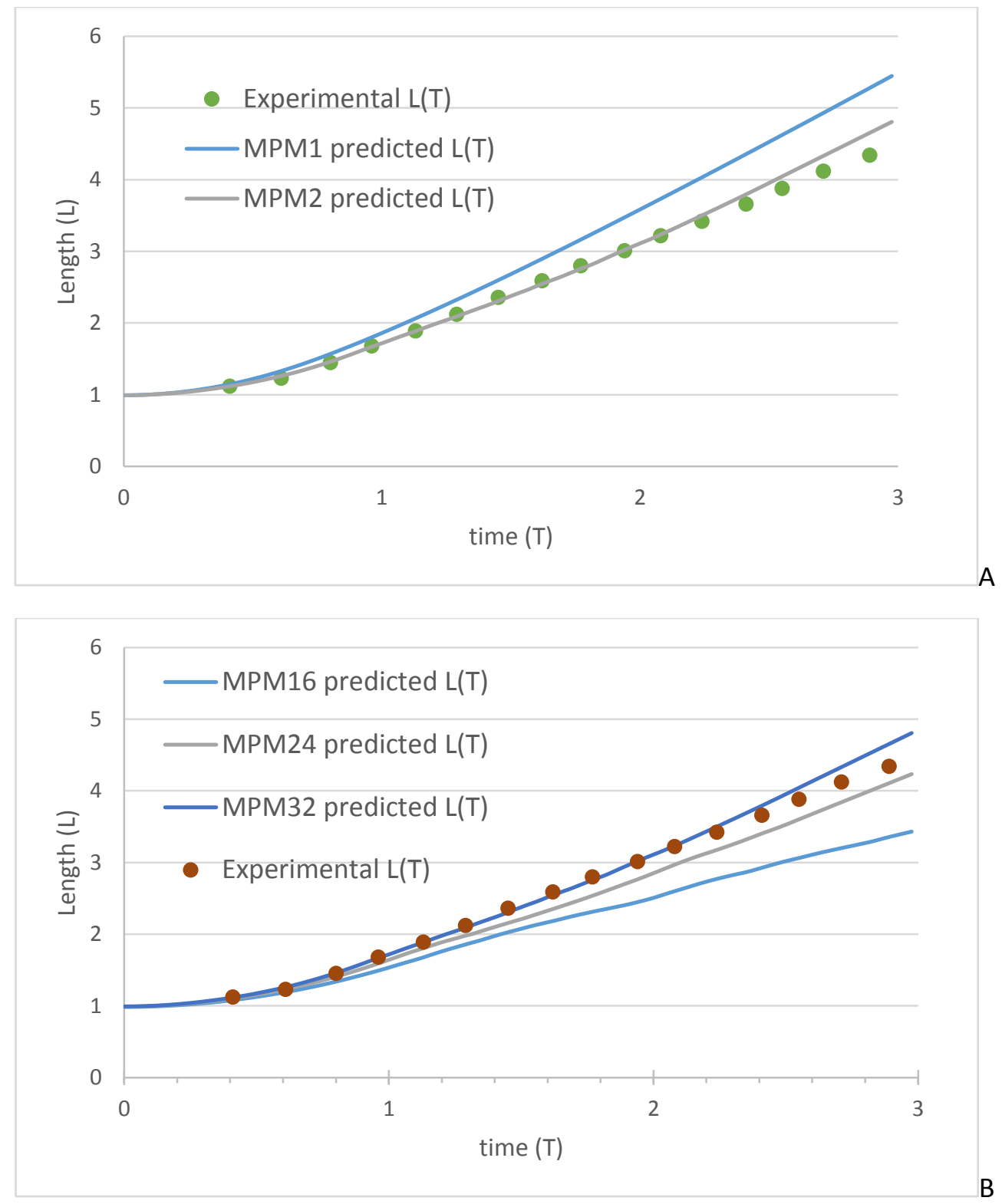

Figure 10.3 MPM simulated water wave front and experimental data for dam breakage model. 

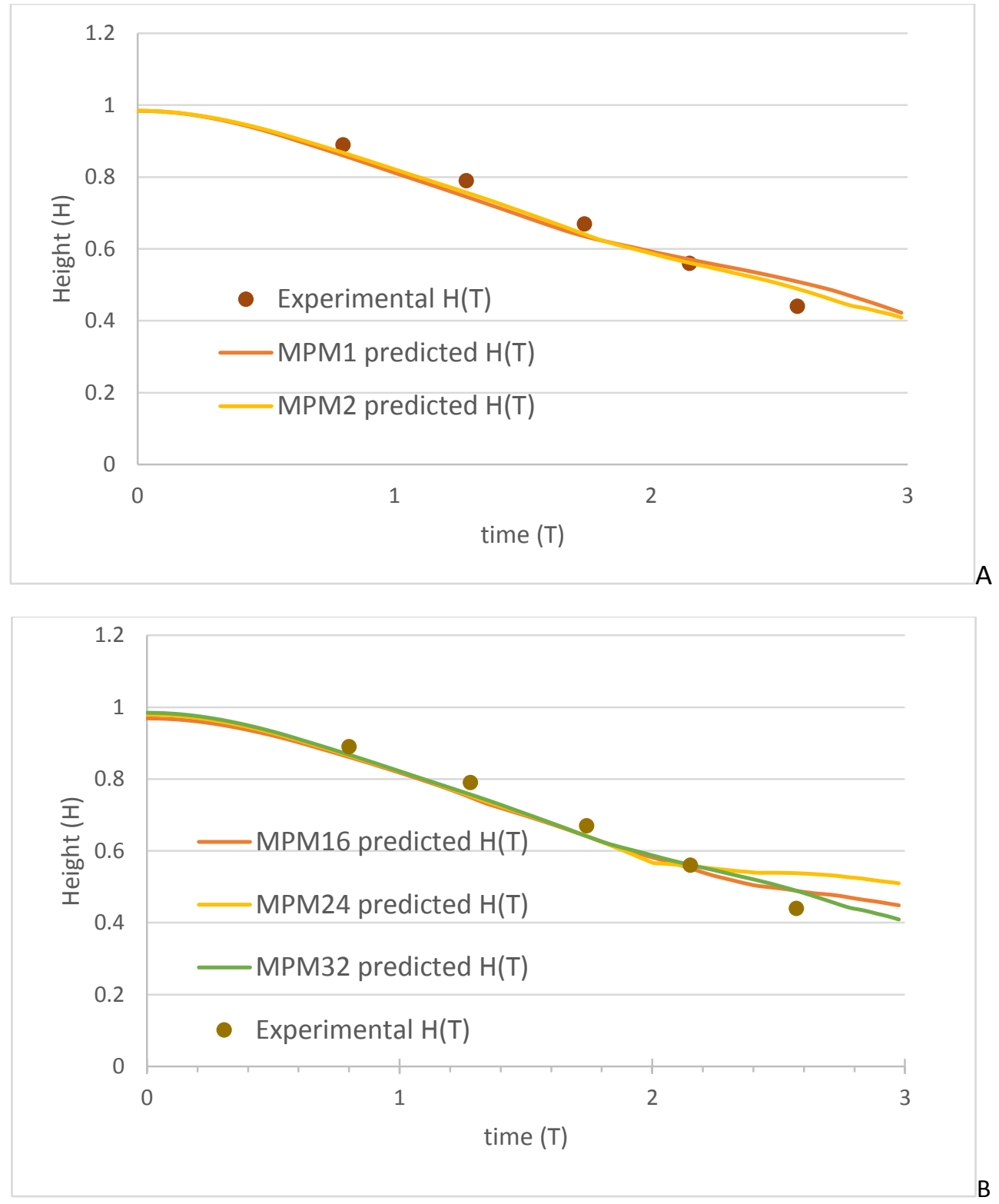

Figure 10.4 MPM simulated water column height and experimental data for dam breakage model. 

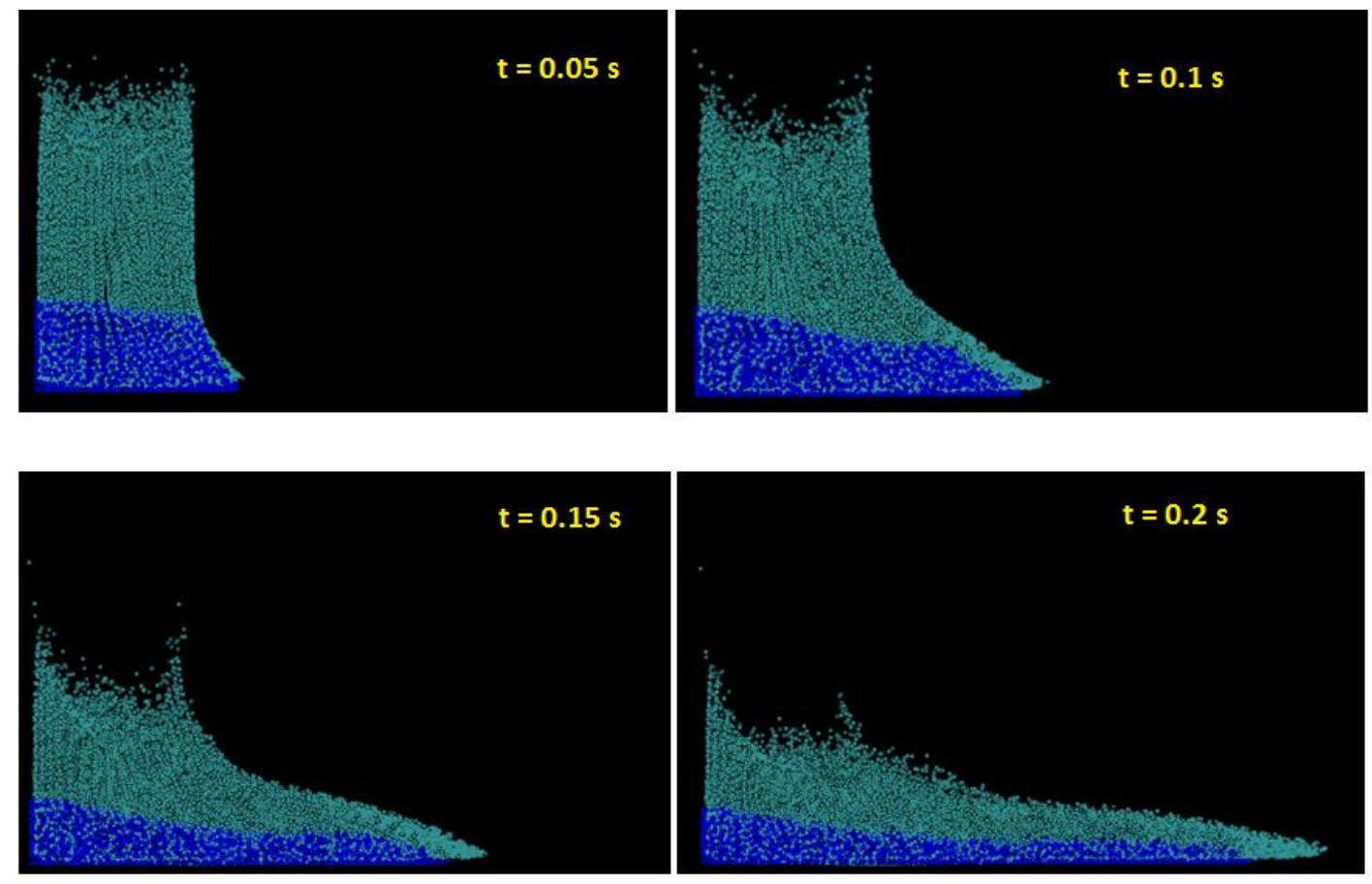

Figure 10.5 Simulation of two-phase flow in a tank, 4 snapshots from numerical modeling. The dark blue dots stand for the glass beads, while light blue dots for water. This figure is prepared by using VMD. (Humphrey, Dalke et al. 1996) 

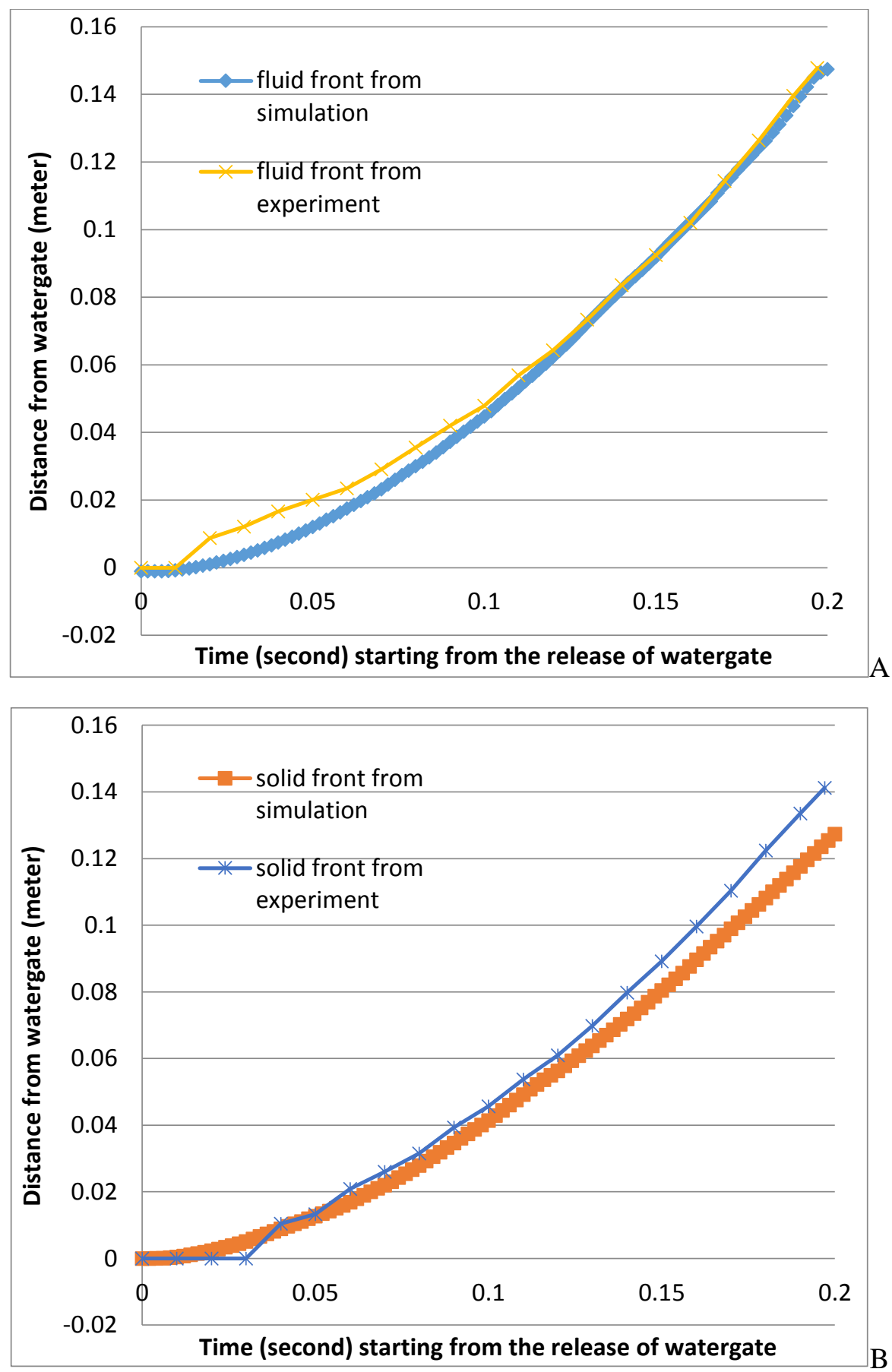

Figure 10.6 Comparison between numerical results and experimental data for two-phase flow. A) Displacement of the liquid phase; B) Displacement of the solid phase. 

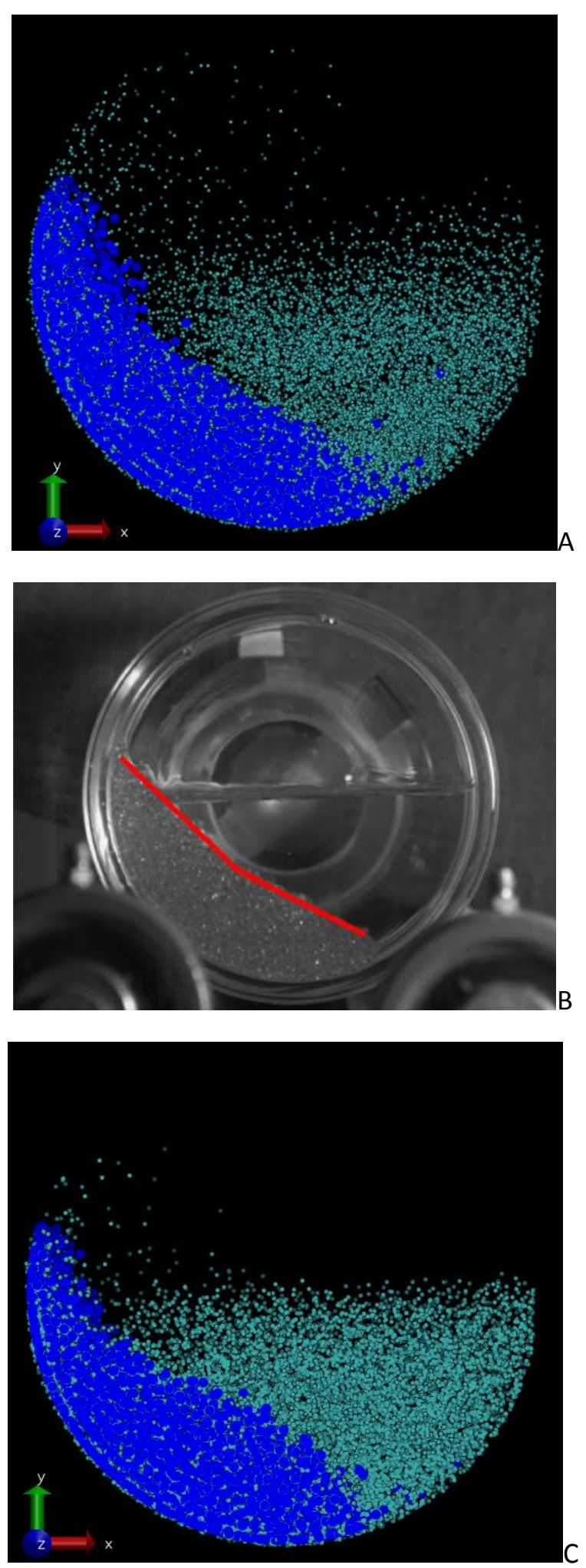

Figure 10.7 Comparison between numerical results and physical experiment for rotating jar. The dark blue dots stand for the glass beads, while light blue dots for water. A) Model with non-slip boundary condition; B) Stable state of beads and water in a rotating jar in physical experiment; C) Models with $50 \%$ of sticky boundary condition. This figure is prepared by using VMD. (Humphrey, Dalke et al. 1996) 


\section{Chapter 11. Concluding Remarks and Future Work}

In this study, the combined DEM-MPM method is proposed and developed for the simulation of multiphase flow. By applying Newton's law of motion on the fluid phase, we derive the equation of momentum conservation, which is consistent with the existing equation, but our derivation is much shorter and has fewer assumptions. The motion of the fluid and the interaction between the solid phase and fluid are simulated using the MPM method, while the contact forces between solid particles and solid structure are calculated using the DEM. Due to the nature of the MPM, the free surface of the fluid is naturally formed without appealing to any other techniques.

The proposed method is verified and validated using physical experimental data and similar numerical tests. The reduced form of model is verified in a water flow experiment, and the full scale solid-liquid flow is validated in a water-glass beads mixture flow test. The dynamic behaviors of fluid head is well reproduced in the numerical modeling. The quantitative comparison shows consistency with previous numerical modeling and with physical experiments. It also correctly demonstrates the influence of spatial resolution and boundary condition on the fidelity of numerical simulation. Thus, we have shown that the combined DEM-MPM method is an effective approach for the simulation of solid-liquid flow. Fine particles can be treated as a kind of viscous fluid, thus, this method has the potential to solve fine particle comminution. 


\section{Bibliography}

Allmen, M. V. (1976). "Laser drilling velocity in metals." J. Applied Physics 47: 5460-5463.

Anderson, T. B. and R. Jackson (1967). "A fluid mechanical description of fluidized beds: Equations of motion." Industrial and Engineering Chemistry Fundamentals 6(4): 527-539.

Armon, E., M. Hill, I. J. Spalding and Y. Zvirin (1989). "Metal drilling with a CO2 laser beam. II. Analysis of aluminum drilling experiments." J. Applied Physics 65(12): 5003-5006.

Armon, E., Y. Zvirin, G. Laufer and A. Solan (1989). "Metal drilling with a CO2 laser beam. I. Theory." J. Applied Physics 65(12): 4995-5002.

Bandara, S. and K. Soga (2015). "Coupling of soil deformation and pore fluid flow using material point method." Computers and Geotechnics 63: 199-214.

Bellantone, R. and R. K. Ganesh (1991). Analytical model for laser hold drilling final report: Contract II. E. H. report submitted to Pratt and Whitney Aircraft, CT.

Belytschko, T., Y. Guo, W. K. Liu and S. P. Xiao (2000). "A unified stability analysis of meshless particle methods." International Journal for Numerical Methods in Engineering 48(9): 1359-1400.

Brennen, C. E. (2005). Fundamentals of Multiphase Flows, Cambridge University Press.

Brown, G. J. (2002). "Erosion prediction in slurry pipeline tee-junctions." Applied Mathematical Modelling 26(2): 155-170.

Burden, D. and R. Faires (2012). Numerical Analysis, Thomson Brooks/Cole.

Buzzi, O., D. M. Pedroso and A. GiacomInl (2008). "Caveats on the implementation of the generalized material point method." CMES - Computer Modeling in Engineering and Sciences 31(2): 85-106.

Campbell, C. S. (1986). COMPUTER SIMULATION OF RAPID GRANULAR FLOWS. Proceedings of the U.S. National Congress of Applied Mechanics.

Chan, C. L. and J. Mazumder (1987). "One-dimensional steady-state model for damage by vaporization and liquid expulsion due to laser-material interaction." Journal of Applied Physics 62(11): 4579-4586.

Chan, C. L. and J. Mazumder (1987). "One-dimensional steady-state model for damage by vaporization and liquid expulsion due to laser-material interaction." J. Applied Physics 62(11): 4579-4586.

Chen, Z., L. Shen, Y. W. Mai and Y. G. Shen (2005). "A bifurcation-based decohesion model for simulating the transition from localization to decohesion with the MPM." Zeitschrift fur Angewandte Mathematik und Physik 56(5): 908-930.

Chu, K. W., S. B. Kuang, A. B. Yu and A. Vince (2012). "Particle scale modelling of the multiphase flow in a dense medium cyclone: Effect of fluctuation of solids flowrate." Minerals Engineering 33: 34-45. 
Cleary, P. W. (2000). "DEM simulation of industrial particle flows: Case studies of dragline excavators, mixing in tumblers and centrifugal mills." Powder Technology 109(1-3): 83-104.

Cleary, P. W. (2001). "Charge behaviour and power consumption in ball mills: Sensitivity to mill operating conditions, liner geometry and charge composition." International Journal of Mineral Processing 63(2): 79-114.

Cleary, P. W. (2006). "Axial transport in dry ball mills." Applied Mathematical Modelling 30(11): 13431355.

Cleary, P. W. (2015). "Prediction of coupled particle and fluid flows using DEM and SPH." Minerals Engineering 73: 85-99.

Cleary, P. W. and R. D. Morrison (2011). "Predicting patterns of slurry flow within and discharge from a 3D pilot SAG mill." Proc. SAG 2011.

Cleary, P. W. and R. D. Morrison (2012). "Prediction of 3D slurry flow within the grinding chamber and discharge from a pilot scale SAG mill." Minerals Engineering 39: 184-195.

Cleary, P. W., M. Sinnott and R. Morrison (2006). "Prediction of slurry transport in SAG mills using SPH fluid flow in a dynamic DEM based porous media." Minerals Engineering 19(15): 1517-1527.

Cleary, P. W., M. D. Sinnott and R. D. Morrison (2008). "DEM prediction of particle flows in grinding processes." International Journal for Numerical Methods in Fluids 58(3): 319-353.

Cleary, P. W., M. D. Sinnott and R. D. Morrison (2009). "Separation performance of double deck banana screens - Part 1: Flow and separation for different accelerations." Minerals Engineering 22(14): 12181229.

Collins, J. and P. Gremaud (2011). "A simple model for laser drilling." Mathematics and Computers in Simulation 81(8): 1541-1552.

Cundall, P. A. and O. D. L. Strack (1979). "A discrete numerical model for granular assemblies." Geotechnique 29(1): 47-65.

Dehnen, W. and H. Aly (2012). "Improving convergence in smoothed particle hydrodynamics simulations without pairing instability." Monthly Notices of the Royal Astronomical Society 425(2): 1068-1082.

Djordjevic, N. (2003). "Discrete element modelling of the influence of lifters on power draw of tumbling mills." Minerals Engineering 16(4): 331-336.

Djordjevic, N. (2005). "Influence of charge size distribution on net-power draw of tumbling mill based on DEM modelling." Minerals Engineering 18(3): 375-378.

Ergun, S. (1952). "Fluid flow through packed columns." Chemical Engineering and Processing 48(2): 8994.

Evans, L. C. (1997). Partial Differential Equations, American Mathematics Society. 19: 662. 
Faghri, A., Y. Zhang and J. R. Howell (2010). Advanced Heat and Mass Transfer. Columbia, MO, Global Digital Press.

Fernandez, J. W., P. W. Cleary, M. D. Sinnott and R. D. Morrison (2011). "Using SPH one-way coupled to DEM to model wet industrial banana screens." Minerals Engineering 24(8): 741-753.

Ferrari, A. (2010). "SPH simulation of free surface flow over a sharp-crested weir." Advances in Water Resources 33(3): 270-276.

Gan, Y., Z. Chen and S. Montgomery-Smith (2011). "Improved material point method for simulating the zona failure response in piezo-assisted intracytoplasmic sperm injection." CMES - Computer Modeling in Engineering and Sciences 73(1): 45-75.

Ganesh, R. K., A. Faghri and Y. Hahn (1997). "A generalized thermal modeling for laser drilling process - I. Mathematical modeling and numerical methodology." Int. J. Heat Mass Transfer 40(14): 3351-3360.

Gao, D. and J. A. Herbst (2009). "Alternative ways of coupling particle behaviour with fluid dynamics in mineral processing." International Journal of Computational Fluid Dynamics 23(2): 109-118.

Gnanavelu, A., N. Kapur, A. Neville, J. F. Flores and N. Ghorbani (2011). "A numerical investigation of a geometry independent integrated method to predict erosion rates in slurry erosion." Wear 271(5-6): 712-719.

Guzel, B., M. Prakash, S. E. Semercigil and Ö. F. Turan (2005). Energy dissipation with sloshing for absorber design. American Society of Mechanical Engineers, Dynamic Systems and Control Division (Publication) DSC.

Ha, J. and P. W. Cleary (2005). "Simulation of high pressure die filling of a moderately complex industrial object using smoothed particle hydrodynamics." International Journal of Cast Metals Research 18(2): 8192.

Han, L. and F. W. Liou (2004). "Numerical investigation of the influence of laser beam mode on melt pool." International Journal of Heat and Mass Transfer 47(19-20): 4385-4402.

Herbst, J. A. and L. Nordell (2001). "Optimization of the design of sag mill internals using high fidelity simulation." Proceedings of the SAG Conference 4: 150-164.

Herbst, J. A. and A. V. Potapov (2004). "Radical innovations in mineral processing simulation." Minerals and Metallurgical Processing 21(2): 57-64.

Hilton, J. E. and P. W. Cleary (2011). "The influence of particle shape on flow modes in pneumatic conveying." Chemical Engineering Science 66(3): 231-240.

Hilton, J. E., L. R. Mason and P. W. Cleary (2010). "Dynamics of gas-solid fluidised beds with nonspherical particle geometry." Chemical Engineering Science 65(5): 1584-1596.

Ho, C. Y. and J. K. Lu (2003). "A closed form solution for laser drilling of silicon nitride and alumina ceramics." Journal of Materials Processing Technology 140(1-3 SPEC.): 260-263. 
Hoomans, B. P. B., J. A. M. Kuipers and W. P. M. Van Swaaij (2000). "Granular dynamics simulation of segregation phenomena in bubbling gas- fluidised beds." Powder Technology 109(1-3): 41-48.

Humphrey, W., A. Dalke and K. Schulten (1996). "VMD - Visual Molecular Dynamics." J. Molec. Graphics 14: 33-38.

Jayasundara, C. T., R. Y. Yang, A. B. Yu and D. Curry (2008). "Discrete particle simulation of particle flow in IsaMill-Effect of grinding medium properties." Chemical Engineering Journal 135(1-2): 103-112.

Jiang, S., Z. Chen, T. D. Sewell and Y. Gan (2015). "Multiscale simulation of the responses of discrete nanostructures to extreme loading conditions based on the material point method." Computer Methods in Applied Mechanics and Engineering 297: 219-238.

Jin, Z., Y. Yuan and M. Song (1992). Interior Ballistics, Beijing Institute of Technology Press.

Kafui, D. K. and C. Thornton (2008). "Fully-3D DEM simulation of fluidised bed spray granulation using an exploratory surface energy-based spray zone concept." Powder Technology 184(2): 177-188.

Kafui, K. D., C. Thornton and M. J. Adams (2002). "Discrete particle-continuum fluid modelling of gassolid fluidised beds." Chemical Engineering Science 57(13): 2395-2410.

Kalala, J. T., M. Breetzke and M. H. Moys (2008). "Study of the influence of liner wear on the load behaviour of an industrial dry tumbling mill using the Discrete Element Method (DEM)." International Journal of Mineral Processing 86(1-4): 33-39.

Kar, A. and J. Mazumder (1990). "Two-dimensional model for material damage due to melting and vaporization during laser irradiation." J. Applied Physics 68(8): 3884-3891.

Kar, S. P. and P. Rath (2014). "A fixed-grid based mixture model for pulsed laser phase change process." Computational Thermal Sciences 6(1): 13-26.

Kawaguchi, T., M. Sakamoto, T. Tanaka and Y. Tsuji (2000). "Quasi-three-dimensional numerical simulation of spouted beds in cylinder." Powder Technology 109(1-3): 3-12.

Kawaguchi, T., T. Tanaka and Y. Tsuji (1995). "Numerical simulation of two-dimensional fluidized beds using DEM (The case of spouted bed: Comparison between 2-D model and 3-D model)." Nippon Kikai Gakkai Ronbunshu, B Hen/Transactions of the Japan Society of Mechanical Engineers, Part B 61(589): 3169-3175.

Kawaguchi, T., T. Tanaka and Y. Tsuji (1998). "Numerical simulation of two-dimensional fluidized beds using the discrete element method (comparison between the two- and three-dimensional models)." Powder Technology 96(2): 129-138.

Kendoush, A. A. (1998). "Theory of stagnation region heat and mass transfer to fluid jets impinging normally on solid surfaces." Chem. Eng. Proc. 37(3): 223-228.

Li, J. F., L. Li and F. H. Stott (2004). "A three-dimensional numerical model for a convection-diffusion phase change process during laser melting of ceramic materials." International Journal of Heat and Mass Transfer 47(25): 5523-5539. 
Li, J. G., Y. Hamamoto, Y. Liu and X. Zhang (2014). "Sloshing impact simulation with material point method and its experimental validations." Computers and Fluids 103: 86-99.

Lian, Y., F. Zhang, Y. Liu and X. Zhang (2013). "Material point method and its applications." Advances in Mechanics 43(2): 237-264.

Lichter, J. K., M. Suazo, R. Noriega and V. Murariu (2011). "The application of multiphysics models for the design of mill discharge systems." Int. Autogenous Grinding, Semiautogenous Grinding and High Pressure Grinding Roll Technology 2011 (SAG 2011).

Liu, M., Y. Yang, S. Stein, Y. Zhu and J. Engeln (2000). "Crustal shortening in the Andes: Why do GPS rates differ from geological rates?" Geophysical Research Letters 27(18): 3005-3008.

Liu, P., Y. Liu, X. Zhang and Y. Guan (2015). "Investigation on high-velocity impact of micron particles using material point method." International Journal of Impact Engineering 75: 241-254.

Low, D. K. Y., L. Li and P. J. Byrd (2002). "Hydrodynamic physical modeling of laser drilling." Journal of Manufacturing Science and Engineering, Transactions of the ASME 124(4): 852-862.

Mackenzie-Helnwein, P., P. Arduino, W. Shin, J. A. Moore and G. R. Miller (2010). "Modeling strategies for multiphase drag interactions using the material point method." International Journal for Numerical Methods in Engineering 83(3): 295-322.

Mao, S. (2013). Material point method and adaptive meshing applied to fluid-structure interaction (FSI) problems. American Society of Mechanical Engineers, Fluids Engineering Division (Publication) FEDSM.

Martin, J. and W. Moyce (1952). "An experimental study of the collapse of liquid columns on a rigid horizontal plane." Phil. Trans. Roy. Soc. Lond. 244: 312-324.

Mast, C. M., P. Mackenzie-Helnwein, P. Arduino, G. R. Miller and W. Shin (2012). "Mitigating kinematic locking in the material point method." Journal of Computational Physics 231(16): 5351-5373.

Mikami, T., H. Kamiya and M. Horio (1998). "Numerical simulation of cohesive powder behavior in a fluidized bed." Chemical Engineering Science 53(10): 1927-1940.

Mishra, B. K. and R. K. Rajamani (1992). "The discrete element method for the simulation of ball mills." Applied Mathematical Modelling 16(11): 598-604.

Mishra, B. K. and R. K. Rajamani (1994). "Simulation of charge motion in ball mills. Part 1: experimental verifications." International Journal of Mineral Processing 40(3-4): 171-186.

Modest, M. F. (1996). "Three-dimensional, transient model for laser machining of ablating/decomposing materials." International Journal of Heat and Mass Transfer 39(2): 221-234.

Momin, O., S. Z. Shuja and B. S. Yilbas (2012). "Laser heating of titanium and steel: Phase change at the surface." International Journal of Thermal Sciences 54: 230-241.

Monaghan, J. J. (1988). "An introduction to SPH." Computer Physics Communications 48(1): 89-96. 
Monaghan, J. J. (1992). "Smoothed particle hydrodynamics." Annual Review of Astronomy and Astrophysics 30(1): 543-574.

Ng, G. K. L., P. L. Crouse and L. Li (2006). "An analytical model for laser drilling incorporating effects of exothermic reaction, pulse width and hole geometry." International Journal of Heat and Mass Transfer 49(7-8): 1358-1374.

Nguyen, V. P., T. Rabczuk, S. Bordas and M. Duflot (2008). "Meshless methods: A review and computer implementation aspects." Mathematics and Computers in Simulation 79(3): 763-813.

NSF (2006). Simulation-Based Engineering Science: Revolutionizing Engineering Science through Simulation. Washington, DC.

Oberkampf, W. L. and F. G. Blottner (1998). "Issues in computational fluid dynamics code verification and validation." AIAA Journal 36(5): 687-695.

Parandoush, P. and A. Hossain (2014). "A review of modeling and simulation of laser beam machining." International Journal of Machine Tools and Manufacture 85: 135-145.

Pastras, G., A. Fysikopoulos, P. Stavropoulos and G. Chryssolouris (2014). "An approach to modelling evaporation pulsed laser drilling and its energy efficiency." International Journal of Advanced Manufacturing Technology 72(9-12): 1227-1241.

Potapov, A. V., M. L. Hunt and C. S. Campbell (2001). "Liquid-solid flows using smoothed particle hydrodynamics and the discrete element method." Powder Technology 116(2-3): 204-213.

Powell, M. S., N. S. Weerasekara, S. Cole, R. D. Laroche and J. Favier (2011). "DEM modelling of liner evolution and its influence on grinding rate in ball mills." Minerals Engineering 24(3-4): 341-351.

Prakash, M., P. W. Cleary, J. Ha, M. N. Noui-Mehidi, H. M. Blackburn and G. Brooks (2007). "Simulation of suspension of solids in a liquid in a mixing tank using SPH and comparison with physical modelling experiments." Progress in Computational Fluid Dynamics 7(2-4): 91-100.

Rajamani, R., D. Alkac, J. Delgadillo, P. Kumar, D. Page, M. Fillion and S. Pelletier (2011). "Pulp-lifter flow modelling study in a pilot scale mill and application to plant scale mills." Int. Autogenous Grinding, Semiautogenous Grinding and High Pressure Grinding Roll Technology 2011 (SAG 2011).

Rajamani, R. K. and B. K. Mishra (1996). "Dynamics of ball and rock charge in sag mills." Proc. SAG 1996.

Robinson, M., M. Ramaioli and S. Luding (2014). "Fluid-particle flow simulations using two-way-coupled mesoscale SPH-DEM and validation." International Journal of Multiphase Flow 59: 121-134.

Rohsenow, W., J. Hartnett and Y. Cho (1998). Handbook of Heat Transfer. New York, McGraw-Hill Education.

Semak, V. and A. Matsunawa (1997). "The role of recoil pressure in energy balance during laser materials processing." Journal of Physics D: Applied Physics 30(18): 2541-2552. 
Semak, V. V. and T. F. Miller (2013). "Simulation of laser penetration efficiency." Journal of Physics D: Applied Physics 46(38).

Shao, J. R., H. Q. Li, G. R. Liu and M. B. Liu (2012). "An improved SPH method for modeling liquid sloshing dynamics." Computers and Structures 100-101: 18-26.

Shen, Z. H., S. Y. Zhang, J. Lu and X. W. Ni (2001). "Mathematical modeling of laser induced heating and melting in solids." Optics and Laser Technology 33(8): 533-537.

Shidfar, A., M. Alinejadmofrad and M. Garshasbi (2009). "A numerical procedure for estimation of the melt depth in laser material processing." Optics and Laser Technology 41(3): 280-284.

Shuja, S. Z. and B. S. Yilbas (2011). "Laser produced melt pool: Influence of laser intensity parameter on flow field in melt pool." Optics and Laser Technology 43(4): 767-775.

Sinnott, M., P. W. Cleary and R. Morrison (2006). "Analysis of stirred mill performance using DEM simulation: Part 1- Media motion, energy consumption and collisional environment." Minerals Engineering 19(15): 1537-1550.

Sinnott, M., P. W. Cleary and R. D. Morrison (2011). "Slurry flow in a tower mill." Minerals Engineering 24(2): 152-159.

Solana, P., P. Kapadia, J. Dowden, W. S. O. Rodden, S. S. Kudesia, D. P. Hand and J. D. C. Jones (2001). "Time dependent ablation and liquid ejection processes during the laser drilling of metals." Optics Communications 191(1-2): 97-112.

Solnordal, C. B., C. Y. Wong, A. Zamberi, M. Jadid and Z. Johar (2013). "Determination of erosion rate characteristic for particles with size distributions in the low Stokes number range." Wear 305(1-2): 205215.

Sulsky, D., Z. Chen and H. L. Schreyer (1994). "A particle method for history-dependent materials." Computer Methods in Applied Mechanics and Engineering 118(1-2): 179-196.

Sun, R. and H. Xiao (2015). "Diffusion-based coarse graining in hybrid continuum-discrete solvers: Applications in CFD-DEM." International Journal of Multiphase Flow 72: 233-247.

Sun, X., M. Sakai and Y. Yamada (2013). "Three-dimensional simulation of a solid-liquid flow by the DEMSPH method." Journal of Computational Physics 248: 147-176.

Tartakovsky, A. M. and P. Meakin (2006). "Pore scale modeling of immiscible and miscible fluid flows using smoothed particle hydrodynamics." Advances in Water Resources 29(10): 1464-1478.

Tran, L. T., J. Kim and M. Berzins (2010). "Solving time-dependent PDEs using the material point method, a case study from gas dynamics." International Journal for Numerical Methods in Fluids 62(7): 709-732.

Tsuji, T., K. Yabumoto and T. Tanaka (2008). "Spontaneous structures in three-dimensional bubbling gasfluidized bed by parallel DEM-CFD coupling simulation." Powder Technology 184(2): 132-140. 
Tsuji, Y., T. Kawaguchi and T. Tanaka (1993). "Discrete particle simulation of two-dimensional fluidized bed." Powder Technology 77(1): 79-87.

Weerasekara, N. S., M. S. Powell, P. W. Cleary, L. M. Tavares, M. Evertsson, R. D. Morrison, J. Quist and R. M. Carvalho (2013). "The contribution of DEM to the science of comminution." Powder Technology 248: 3-4.

Wen, C. Y. and Y. H. Yu (1966). "Mechanics of fluidization." A.I.Ch.E. Series 62: 100-111.

Xu, B. H. and A. B. Yu (1997). "Numerical simulation of the gas-solid flow in a fluidized bed by combining discrete particle method with computational fluid dynamics." Chemical Engineering Science 52(16): 2785-2809.

Yang, R. Y., C. T. Jayasundara, A. B. Yu and D. Curry (2006). "DEM simulation of the flow of grinding media in IsaMill." Minerals Engineering 19(10): 984-994.

Yang, Y., Z. Chen and Y. Zhang (2016). "Melt flow and heat transfer in laser drilling." International Journal of Thermal Sciences 107: 141-152.

Yang, Y. and M. Liu (2009). "Crustal thickening and lateral extrusion during the Indo-Asian collision: A 3D viscous flow model." Tectonophysics 465(1-4): 128-135.

Yang, Y. and M. Liu (2013). "The indo-asian continental collision: A 3-D viscous model." Tectonophysics 606: 198-211.

Yang, Y., P. Sun and Z. Chen (2017). "Combined MPM-DEM for simulating the interaction between solid elements and fluid particles." Communications in Computational Physics.

York Ii, A. R., D. Sulsky and H. L. Schreyer (2000). "Fluid-membrane interaction based on the material point method." International Journal for Numerical Methods in Engineering 48(6): 901-924.

Yu, A. B. and B. H. Xu (2003). "Particle scale modelling of gas-solid flow in fluidisation." J. Chem. Technol. Biotechnol. 7: 305-317.

Zeng, D., W. P. Latham and A. Kar (2005). "Two-dimensional model for melting and vaporization during optical trepanning." Journal of Applied Physics 97(10).

Zhang, D. Z., Q. Zou, W. B. VanderHeyden and X. Ma (2008). "Material point method applied to multiphase flows." Journal of Computational Physics 227(6): 3159-3173.

Zhang, H. W., K. P. Wang and Z. Chen (2009). "Material point method for dynamic analysis of saturated porous media under external contact/impact of solid bodies." Computer Methods in Applied Mechanics and Engineering 198(17-20): 1456-1472.

Zhang, W., Y. L. Yao and K. Chen (2001). "Modelling and analysis of UV laser micromachining of copper." Int. J. Adv. Manuf. Technol 18(5): 323-331.

Zhang, X., K. Y. Sze and S. Ma (2006). "An explicit material point finite element method for hypervelocity impact." International Journal for Numerical Methods in Engineering 66(4): 689-706. 
Zhang, Y. and A. Faghri (1999). "Vaporization, melting and heat conduction in the laser drilling process." Int. J. Heat Mass Transfer 42(10): 1775-1790.

Zhang, Y., E. P. Reuterfors, B. S. McLaury, S. A. Shirazi and E. F. Rybicki (2007). "Comparison of computed and measured particle velocities and erosion in water and air flows." Wear 263(1-6 SPEC. ISS.): 330-338.

Zhang, Y., Z. Shen and X. Ni (2014). "Modeling and simulation on long pulse laser drilling processing." International Journal of Heat and Mass Transfer 73: 429-437.

Zhu, H. P., Z. Y. Zhou, R. Y. Yang and A. B. Yu (2007). "Discrete particle simulation of particulate systems: Theoretical developments." Chemical Engineering Science 62(13): 3378-3396. 


\section{VITA}

Youqing Yang was born in 1964 in Fuping County, Shaanxi, China. In 1979, he was admitted to Xi'an Aeronautical School of Technology where he completed a major in Mechanical Engineering with a focus on hydraulic systems, graduating in 1983 . He received his B. S. in Seismology and Geology from Peking University in 1989. He earned his M.S. in Geodynamics from Peking University in 1992 and a Ph. D in Geophysics from the Institute of Geology, State Seismological Bureau of China. In 2016, he received a Ph. D in Structural Engineering from the University of Missouri.

His career started as a mechanical engineer at Aircraft Structural Institute. After graduation from Peking University, he worked on oil reservoir simulation, geological structure analysis, plate tectonics, geodynamics in Geology Institute of China State Seismological Bureau, Geological Sciences department at University of Missouri. He is currently working at ME Global Incorporation as a senior mechanical engineer in charge of modeling and simulation for mill structural optimization.

He is married to Hongyan Liu and has two sons named Bowen Yang and Kevin Yang. 